\author{
UNIVERSIDADE DE SÃO PAULO \\ ESCOLA DE ENGENHARIA DE SÃO CARLOS \\ DEPARTAMENTO DE ENGENHARIA DE ESTRUTURAS
}

\title{
ANÁLISE DO PROBLEMA HARMÔNICO DE RADIAÇÃO E DIFUSÃO ACÚSTICA, USANDO O MÉTODO DOS ELEMENTOS DE CONTORNO
}

\author{
Marcelo Greco
}

Dissertação apresentada à Escola de Engenharia de São Carlos, da Universidade de São Paulo, como parte dos requisitos para obtenção do título de Mestre em Engenharia de Estruturas.

ORIENTADOR: Professor Titular Wilson Sergio Venturini

São Carlos 
Se enxertaste no coração a rosa do Amor, Não foi inútil a tua vida, pois tanto faz Que tenhas tentado seguir a trilha de Alá, Ou empunhado sem remorsos a taça de vinho.

RUBAIYAT (Omar Khayyam) 
Aos meus pais e a Jisela 


\section{AGRADECIMENTOS}

A Deus, por tudo.

Ao meu irmão Juliano, pela amizade e incentivo;

Às funcionárias do departamento, Lúcia, Suelly e Cida, e ao funcionário Wilson, pela agradável convivência diária e pelo bom trabalho, muitas vezes não percebido;

Às bibliotecárias do departamento, Maria Nadir e Eliana, pela presteza e eficiência no trabalho, além do bom humor e simpatia;

Às funcionárias da secretaria, Rosi e Marta, e ao funcionário Toninho, pela competência no serviço e bom atendimento;

Ao Prof. Dr. Humberto Breves Coda, pelas idéias, paciência, boa vontade de ajudar e principalmente pelo exemplo moral de comportamento;

Ao meu orientador, Prof. Tit. Wilson Sergio Venturini, pelos ensinamentos, apoio e boa orientação;

À fundação governamental brasileira CAPES, Coordenação de Aperfeiçoamento de Pessoal de Nível Superior, pela bolsa de mestrado, sem a qual não seria possível a realização deste trabalho;

Ao Prof. Tit. José Elias Laier, por ter participado das minhas bancas de qualificação e defesa de mestrado, colaborando com preciosas sugestões;

Aos Professores Euclides de Mesquita Neto e Paulo Sergio Varoto, pela participação e colaboração nas minhas bancas de defesa e qualificação de mestrado, respectivamente;

Ao Prof. Mario Mourelle Perez, do Departamento de Engenharia Mecânica da Universidade Federal de Uberlândia, e ao Prof. Saulo Faria Almeida Barretto, do Núcleo de Pesquisas Tecnológicas da Universidade de Mogi das Cruzes, pelos artigos e trabalhos, que foram valiosos materiais de consulta;

Aos Professores Francisco Antonio Romero Gesualdo e Mauro Prudente, pelas cartas de recomendação que me ajudaram a ingressar no programa de mestrado do Departamento de Engenharia de Estruturas na EESC-USP;

Aos bons amigos feitos no departamento. 


\title{
SUMÁRIO
}

\author{
LISTA DE FIGURAS
}

LISTA DE TABELAS

LISTA DE ABREVIATURAS E SIGLAS

LISTA DE SÍMBOLOS

RESUMO

ABSTRACT

INTRODUÇÃO

CAPÍTULO 1 - FORMULAÇÃO DAS EQUAÇÕES FUNDAMENTAIS 3 DA ACÚSTICA

1.1 Revisão bibliográfica 3

1.2 Conceitos básicos $\quad 7$

1.2.1 Equação de estado

1.2.2 Equação de continuidade $\quad 7$

1.2.3 Equação de Euler 9

1.2.4 Equação transiente de ondas 11

1.2.5 Equação harmônica de ondas (Equação de Helmholtz) 13

CAPÍTULO 2 - O MÉTODO DOS ELEMENTOS DE CONTORNO 14 APLICADO A PROBLEMAS BIDIMENSIONAIS GOVERNADOS PELA EQUAÇÃO DE HELMHOLTZ

2.1 Núcleos de pressão acústica $\quad 14$

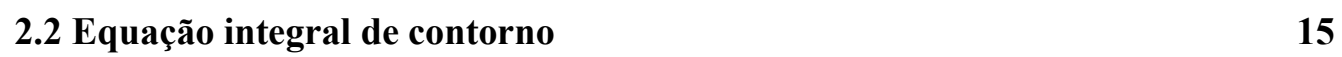

2.2.1 Equacionamento básico 15 
2.3 Representação matricial do MEC $\quad 19$

2.3.1 Generalidades $\quad 19$

2.3.2 Sistema de equações algébricas $\quad 20$

2.4 Técnicas de integração $\quad 24$

2.4.1 Integração numérica $\quad 24$

2.4.2 Ponto de colocação quase singular $\quad 26$

2.4.3 Sub-elementação $\quad 27$

2.4.4 Ponto de colocação no contorno 29

2.4.5 Aplicação do CHIEF $\quad 32$

2.5 Potenciais de pressão em pontos internos ao domínio 34

2.6 Tipos de termos de domínio considerados $\quad 34$

CAPÍTULO 3 - EXEMPLOS NUMÉRICOS DE PROBLEMAS DE 36 RADIAÇÃO E DIFUSÃO ACÚSTICA BIDIMENSIONAL

3.1 Considerações iniciais $\quad 36$

$\begin{array}{ll}3.2 & \text { Exemplo } 1 \\ 3.3 & 37\end{array}$

3.3 Exemplo 2 38

3.4 Exemplo 3

3.5 Exemplo 4

3.6 Exemplo 5 40

3.7 Exemplo 6 42

3.8 Exemplo 7 45

3.9 Exemplo 8 46

CAPÍTULO 4 - O MÉTODO DOS ELEMENTOS DE CONTORNO 50 APLICADO A PROBLEMAS ELASTODINÂMICOS BIDIMENSIONAIS NO DOMÍNIO DA FREQÜÊNCIA

4.1 Relações básicas $\quad 50$

4.2 Equação integral de contorno $\quad 53$

4.3 Representação matricial $\quad 55$

4.4 Exemplo numérico $\quad 56$

CAPÍTULO 5 - ACOPLAMENTO FLUIDO-ESTRUTURA (MEC- 58 MEC)

5.1 Introdução $\quad 58$

5.2 Interação entre o meio acústico e a estrutura $\quad 58$

$\begin{array}{ll}5.3 \text { Sistema acoplado de equações } & 61\end{array}$

$\begin{array}{ll}5.4 \text { Exemplo numérico } & 63\end{array}$ 
CONCLUSÕES

ANEXO A - Funções de Bessel utilizadas $\quad 68$

A.1 Funções de Hankel de $1^{\text {a }}$ classe $\quad 68$

$\begin{array}{ll}\text { A.2 Funções modificadas de Bessel } & \mathbf{7 0}\end{array}$

ANEXO B - Fluxograma do acoplamento fluido-estrutura 71

ANEXO C - Distância de um ponto à reta diretriz de um elemento $\quad 80$ linear de contorno

$\begin{array}{ll}\text { ANEXO D - Noções sobre notação indicial } & 81\end{array}$

ANEXO E - Transformação de coordenadas $\quad 83$

REFERÊNCIAS BIBLIOGRÁFICAS $\quad 84$

$\begin{array}{lr}\text { BIBLIOGRAFIA COMPLEMENTAR } & 88\end{array}$

APÊNDICE 


\section{LISTA DE FIGURAS}

Figura 1.1 - Fluxo de massa na direção X do volume infinitesimal .............................. 7

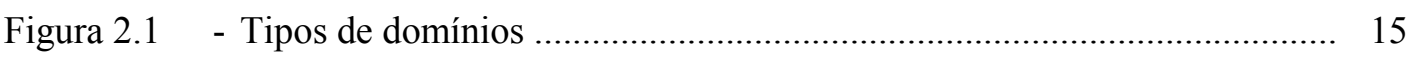

Figura 2.2 - Distribuição constante das variáveis de contorno ................................... 19

Figura 2.3 - Distribuição linear da variável potencial de pressão acústica no

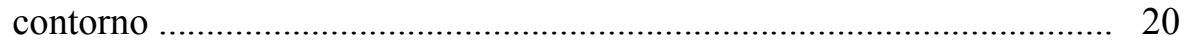

Figura 2.4 - Exemplo de nó descontínuo ……........................................................ 20

Figura 2.5 - Corpo discretizado em elementos lineares de contorno ......................... 21

Figura 2.6 - Pontos de colocação no caso de elemento com distribuições lineares ..... 23

Figura 2.7 - Pontos de colocação posicionados fora do domínio ................................. 26

Figura 2.8 - Representação gráfica das variáveis geométricas envolvidas na integração .................................................................................. 27

Figura 2.9 - Posições do ponto de colocação em relação ao elemento ........................ 27

Figura 2.10 - Limites de integração de um sub-elemento ............................................... 28

Figura 2.11 - Limites adimensionais de integração do elemento e do sub-elemento ..... 28

Figura 2.12 - Coordenadas locais envolvidas na integração singular do elemento com distribuições constantes ....................................................................... $\quad 30$

Figura 2.13 - Coordenadas locais envolvidas na integração singular do elemento com distribuições lineares ............................................................................. 30

Figura 2.14 - Pontos de CHIEF para um domínio infinito .......................................... 33

Figura 2.15 - Núcleos de pressão em um domínio infinito .......................................... 35

Figura 3.1 - Número de ondas $\times$ potencial de pressão (DC_8) …………................. 37

Figura 3.2 - Número de ondas $\times$ potencial de pressão (DC_16) ................................ 38

Figura 3.3 - Número de ondas $\times$ potencial de pressão (DL_8) ................................. 39

Figura 3.4 - Número de ondas $\times$ potencial de pressão (DL_16) ................................. 40

Figura 3.5 - Número de ondas $\times$ fluxo de pressão (DC_16) ...................................... 41

Figura 3.6 - Pontos de CHIEF ……................................................................ 41

Figura 3.7 - Geometria, condições de contorno e pontos de CHIEF ............................ 42 
Figura 3.8 - Pontos internos para o cálculo dos potenciais de pressão 43

Figura 3.9 - Potenciais de pressão obtidos pelo programa ANSYS 43

Figura 3.10 - Fluxos de pressão obtidos pelo programa ANSYS ................................. 43

Figura 3.11 - Distribuição de fluxo de pressão nos lados do quadrado (DL_20) ........... 43

Figura 3.12 - Número de ondas $\times$ fluxo de pressão (DL_16) ...................................... 45

Figura 3.13 - Geometria da barreira acústica no domínio semi-infinito ...................... 46

Figura 3.14 - Simulação do domínio semi-infinito .................................................... 47

Figura 3.15 - Geometria da primeira malha (DL_90) ................................................ 48

Figura 3.16 - Geometria da segunda malha (DL_130) ................................................. 48

Figura 3.17 - Pressões acústicas próximas à barreira no caso da malha DL_90 ........... 49

Figura 3.18 - Pressões acústicas próximas à barreira no caso da malha DL_130 ......... 49

Figura 3.19 - Pressões acústicas na região apresentada nas figuras anteriores, sem a

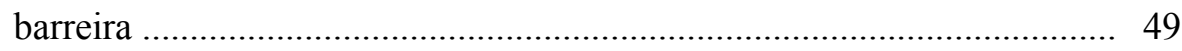

Figura 4.1 - EPD em um ponto no plano do sólido ................................................. 50

Figura 4.2 - Geometria e condições de contorno do exemplo elastodinâmico ............. 56

Figura 5.1 - Sistemas de coordenadas do fluido e da estrutura .................................. 60

Figura 5.2 - Exemplo de acoplamento fluido-estrutura em um elemento de contorno na interface

Figura 5.3 - Geometria, condições de contorno e pontos críticos no contorno do problema

Figura 5.4 - Contornos da malha de elementos finitos utilizada no programa ANSYS 64

Figura 5.5 - Campo de deslocamentos da chapa na direção X [m] ............................. 65

Figura 5.6 - Campo de deslocamentos da chapa na direção Y [m] ............................. 65

Figura 5.7 - Deslocamentos da chapa na direção X [m], obtidos pelo programa ANSYS

Figura 5.8 - Deslocamentos da chapa na direção $\mathrm{Y}$ [m], obtidos pelo programa ANSYS

Figura A.1 - Funções de Bessel do primeiro tipo de ordem 0 e 1 69

Figura A.2 - Funções de Bessel do segundo tipo de ordem 0 e 1 ................................. 69

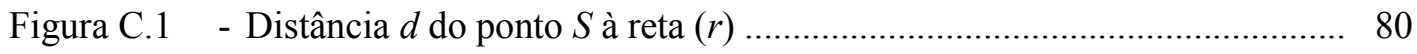

Figura E.2 - Sistemas de coordenadas ................................................................. 83 


\section{LISTA DE TABELAS}

Tabela 3.1 - Tipos de soluções e técnicas de integração ............................................... 36

Tabela 3.2 - Fluxos de pressão nos pontos nodais do quadrado (DL_20) .................... 44

Tabela 3.3 - Potenciais de pressão nos pontos internos .............................................. 44

Tabela 4.1 - Deslocamentos em Y na face A $[\times 0.0000001 \mathrm{~m}]$................................ 57

Tabela 4.2 - Reações de apoio em Y na face B $[\mathrm{N} / \mathrm{m}]$.............................................. 57

Tabela 5.1 - Deslocamentos (UX, UY) em pontos críticos no contorno ...................... 64

Tabela 5.2 - Reações de apoio ............................................................................ 64 


\section{LISTA DE ABREVIATURAS E SIGLAS}

$\begin{array}{ll}\text { MEC } & \text { - Método dos Elementos de Contorno } \\ \text { MEF } & \text { - Método dos Elementos Finitos } \\ \text { EPD } & \text { - Estado Plano de Deformações } \\ \text { EPT } & \text { - Estado Plano de Tensões } \\ \text { SI } & \text { - Sistema Internacional de unidades } \\ \text { CHIEF } & \text { - Combined Helmholtz Integral Equation Formulation } \\ \text { CONDOR } & \text { - Composite Outward Normal Derivative Overlap Relation } \\ \text { RPT } & \text { - Retarded Potential Technique } \\ \text { HGF } & \text { - Helmholtz Gradient Formulation } \\ \text { CHI } & \text { - Coupled Helmholtz Integrals }\end{array}$




\section{LISTA DE SÍMBOLOS}

$p \quad$ - pressão (no problema acústico) ou força de superfície (no problema elástico)

$P \quad$ - pressão interna instantânea no fluido

$P_{0} \quad$ - pressão de equilíbrio no fluido

$\beta$ - módulo volumétrico adiabático

$\rho \quad$ - densidade

$\rho_{0} \quad$ - densidade de equilíbrio no fluido

$S \quad$ - condensação

$\sigma \quad$ - tensão

E - módulo de elasticidade longitudinal

$\varepsilon \quad$ - deformação

$\vec{v} \quad$ - velocidade de partícula

$\vec{u} \quad$ - posição

$\vec{a} \quad$ - aceleração

c - velocidade de propagação da onda acústica.

$\phi \quad$ - potencial de velocidade de onda (meio acústico) ou função de forma (MEC)

$\omega$ - freqüência

$i \quad$ - parte imaginária de um número complexo ou índice ou contador

$K \quad$ - número de ondas

$\Omega \quad$ - domínio

$\Gamma \quad$ - contorno

$\infty \quad$ - infinito

G - taxa de variação de massa no domínio

$\vec{F} \quad$ - Força de corpo por unidade de volume

D - Termos de domínio (ou núcleos de pressão acústica)

$D_{P} \quad$ - Núcleo de pressão concentrado em um ponto 
$D_{L} \quad$ - núcleo de pressão constante distribuído em linha

$\eta \quad$ - direção normal ao contorno

$q$ - fluxo de pressão acústica

$p^{*} \quad$ - solução fundamental do problema acústico

$\delta \quad$ - delta de dirac (solução fundamental) ou delta de Kronecker (notação indicial)

$c_{(S)} \quad$ - termo livre relacionado à posição do ponto de colocação

$R \quad$ - distância entre o ponto de colocação e o ponto de integração

$N E \quad$ - número de elementos

$N N D$ - número de nós

$[H]$ - matriz relacionada à formulação do MEC

$[G]$ - matriz relacionada à formulação do MEC

$\xi \quad$ - coordenada de integração numérica de Gauss num elemento

$L \quad$ - comprimento de um elemento

$\alpha \quad$ - ângulo relacionado a um elemento de contorno

d - distância do ponto de colocação a um elemento de contorno

w $\quad$ - pesos dos pontos de Gauss utilizados nas integrações

$b_{i} \quad$ - força de corpo na direção $i$

$u_{i} \quad$ - deslocamento na direção $i$

$v \quad$ - coeficiente de Poisson

$\lambda \quad$ - constante de Lamé

$\mu \quad$ - constante de Lamé (módulo de elasticidade transversal)

$p_{i} \quad$ - força de superfície na direção $i$

$u_{l m}^{*} \quad$ - solução fundamental do problema elastodinâmico 


\section{RESUMO}

GRECO, M. (2000). Análise do problema harmônico de radiação e difusão acústica, usando o método dos elementos de contorno. São Carlos, 2000. 88p. Dissertação (Mestrado) Escola de Engenharia de São Carlos, Universidade de São Paulo.

Neste trabalho, estudam-se problemas bidimensionais de propagação de ondas acústicas e elásticas, no domínio da freqüência, formulados através do Método dos Elementos de Contorno. A formulação é baseada nas representações integrais das equações diferenciais que governam os fenômenos de propagação de ondas acústicas num meio fluido e de ondas elásticas numa estrutura elástica. Analisa-se também a interação entre o fluido e a estrutura com o uso de sistemas de equações acoplados. As soluções fundamentais utilizadas são expressões exatas e não há necessidade de subdivisão dos domínios em células de integração. São aplicadas técnicas de integração alternativas na escolha das equações algébricas no domínio do fluido, visando a melhora das respostas globais do conjunto. Apresentam-se ainda exemplos numéricos, com o objetivo de possibilitar a modelagem numérica de problemas de acoplamento fluido-estrutura e de radiação e difusão acústica.

Palavras-chave: método dos elementos de contorno; acústica; acoplamento fluido-estrutura. 


\section{ABSTRACT}

GRECO, M. (2000). Harmonic analysis of the acoustic radiation and scattering problems, using boundary element methods. São Carlos, 2000. 88p. Dissertação (Mestrado) - Escola de Engenharia de São Carlos, Universidade de São Paulo.

In this work, acoustic and elastic wave propagation problems in 2D, in frequency domain, are studied and formulated with the Boundary Element Methods. The formulation is based on the integral representations derived from the differential equations that govern the phenomena of acoustic wave propagation in a fluid medium and elastic wave propagation inside an elastic domain. The fluid-structure interaction is also formulated by coupling appropriately the corresponding systems of equations. The fundamental solutions adopted in this work are conveniently chosen to avoid the mass integral terms in the elastic wave integral representation and the equivalent terms in the acoustic integral equation. Thus, the algebraic representations of both problems are written only in terms of boundary values. Subdivisions of the domain to perform integrals over cells are not required. In an attempt to improve the global answers of the fluid problem, several integration techniques have been experimented to build alternative algebraic matrix equations. Numerical examples are presented in order to shown the accuracy of the studied acoustic radiation and scattering problems and also to verify the proposed fluid-structure coupling.

Keywords: boundary element methods; acoustics; fluid-structure coupling. 


\section{INTRODUÇÃO}

O objetivo desta dissertação é fazer uma modelagem numérica, baseada no Método dos Elementos de Contorno (MEC), para problemas bidimensionais de radiação, transmissão e reflexão acústica no domínio da freqüência. O caso bidimensional de estruturas elásticas imersas em fluido sujeito à propagação de ondas acústicas, situação onde ocorre uma interação entre o fluido e a estrutura, também será tema abordado neste trabalho.

Dá-se o nome de acústica à ciência que estuda o fenômeno da propagação de ondas através de um meio fluido compressíve ${ }^{1}$. As ondas estudadas neste trabalho são harmônicas, produzidas por uma fonte cíclica que gera comportamento de resposta cíclico, e longitudinais, as moléculas movimentam-se na direção da propagação da onda. O efeito da gravidade na propagação das ondas é considerado desprezível, portanto a densidade e a pressão de equilíbrio no fluido permanecem constantes. Outras hipóteses adotadas no modelo físico para o fluido são de material isótropo (o comportamento elástico do material é o mesmo em todas as direções), homogêneo (o material apresenta as mesmas propriedades elásticas em qualquer ponto) e perfeitamente elástico (obedece à lei de Hooke e não sofre deformação residual quando submetido à compressão); não são levados em consideração efeitos dissipativos de nenhuma espécie, nem mesmo a influência da temperatura em fenômenos de baixa freqüência.

A propagação de ondas acústicas envolve fenômenos diferentes como radiação (geração de ondas acústicas devido à uma fonte), absorção (dissipação de energi đa da onda acústica), transmissão (transferência de parte da energia de uma onda incidente de um meio para outro) e reflexão (retorno de parte da energia de uma onda incidente entre meios diferentes para o meio de onde se originou a propagação). A radiação acústica pode ser produzida por núcleos de pressão acústica ou corpos ${ }^{3}$ imersos no meio de propagação. A difusão acústica envolve os fenômenos da absorção, transmissão e reflexão. A absorção pode

\footnotetext{
${ }^{1}$ Meio que sofre alteração de volume quando submetido à pressão.

${ }^{2}$ Energia é definida como a capacidade de realizar trabalho.

${ }^{3}$ No caso deste trabalho serão consideradas estruturas elásticas constituídas de material isótropo e homogêneo.
} 
ocorrer devido a perdas de energia no meio de propagação (caso de grandes volumes de fluido) ou devido a perdas causadas por obstáculos (materiais porosos). A transmissão e reflexão são fenômenos intimamente ligados e de acordo com NEPOMUCENO (1977) ocorrem quando as ondas encontram uma interface entre materiais com impedâncias diferentes.

O fenômeno físico da propagação de ondas pode ser referido como som, quando as ondas forem identificadas por seres humanos, ou vibração mecânica, quando o aparelho auditivo humano não for capaz de identificar as ondas. Esta dissertação se limita ao estudo dos fenômenos de radiação, transmissão e reflexão de ondas acústicas, não serão considerados os efeitos dissipativos da absorção. De uma maneira geral, ao longo de toda a dissertação serão utilizados os termos difusão, referindo-se aos apenas aos fenômenos de transmissão e reflexão, e ondas acústicas, que dependendo da freqüêncid 5 podem ser sonoras ou não.

A modelagem numérica a ser utilizada neste trabalho surge da necessidade de resolver problemas físicos, cujas soluções analíticas demandam grande quantidade de tempo e muitas vezes se tornam inviáveis por limitações humanas e do próprio grau de desenvolvimento da ciência. Diante desta dificuldade de solucionar problemas, os métodos numéricos são uma alternativa interessante, e muitas vezes a única, para se chegar a uma resposta aproximada. A aproximação de resultado é uma característica inerente aos métodos numéricos e sua precisão depende de fatores como simplificações de modelo e refinamento de malha (caso dos métodos discretos).

O MEC é um método numérico discreto; o equacionamento é feito em função de elementos discretizados do contorno que definem o domínio do problema. É baseado na representação integral da equação de contorno e é adequado para resolução de problemas em domínios finitos e infinitos, trabalhando com variáveis no contorno. Para o caso de domínios infinitos o método é ideal, pois não requer, a princípio, a discretização do domínio. Outro aspecto importante na utilização do MEC é o estudo da representação integral da equação de Helmholtz, que governa o problema da propagação de ondas, e sua solução fundamental.

A implementação computacional do MEC, aplicado a problemas bidimensionais governados pela equação de Helmholtz, será feita na íntegra e aplicada nos capítulos posteriores na resolução de problemas de propagação de ondas e acoplamento fluidoestrutura.

\footnotetext{
${ }^{4}$ Impedância de um material é definida como a tendência de eliminar movimentos.

${ }^{5}$ Segundo NEPOMUCENO (1977), a faixa de freqüências auditivas varia de 16 a $16000 \mathrm{~Hz}$. Abaixo de $16 \mathrm{~Hz}$ estão os infra-sons e acima de $16000 \mathrm{~Hz}$ tem-se os ultra-sons.
} 


\section{FORMULAÇÃO DAS EQUAÇÕES FUNDAMENTAIS DA ACÚSTICA}

\subsection{Revisão bibliográfica}

A primeira formulação teórica visando a solução numérica de problemas potenciais baseados em equações integrais de contorno foi apresentada por JASWON (1963). O método desenvolvido trata de problemas bidimensionais com operadores harmônico e biharmônico. Na mesma publicação, SYMM (1963) apresentou um trabalho complementar ao de Jaswon que descreve técnicas computacionais destinadas à solução destes problemas, com os resultados obtidos em alguns exemplos. A ampliação do estudo para problemas de elasticidade foi feita por RIZZO (1967), responsável pela formulação direta do Método dos Elementos de Contorno (MEC), na qual as variáveis têm significado físico. Cabe destacar que a formulação direta é a que será utilizada no desenvolvimento deste trabalho, sendo também a mais aplicada atualmente.

Um trabalho pioneiro sobre a solução de problemas de propagação de ondas acústicas transientes, no domínio do tempo, foi apresentado por FRIEDMAN \& SHAW (1962). Neste trabalho apresentou-se uma equação integral para problemas bidimensionais com ondas de choque planas dispersas por obstáculos de seção transversal qualquer.

COPLEY (1967) apresentou uma técnica numérica baseada na representação integral da equação de Helmholtz para problemas harmônicos, no domínio da freqüência, de radiação acústica. Esta técnica utiliza uma relação acústica entre pressão e velocidade de onda normal à superfície. Outro método numérico inovador chamado RPT (Retarded Potential Technique) foi introduzido por MITZNER (1967). Com base na equação integral de potencial no contorno, o autor resolveu problemas de difusão de ondas acústicas incidentes em uma superfície ao longo do tempo.

O primeiro pesquisador a identificar problemas de não unicidade e não existência de resposta em soluções numéricas de problemas harmônicos de radiação acústica formulados através de equações integrais, para determinadas freqüências, foi COPLEY (1968). 
Para resolver o problema da não unicidade de resposta observado por Copley, SCHENCK (1968) propõe uma técnica chamada de CHIEF (Combined Helmholtz Integral Equation Formulation). A técnica consiste na utilização da equação integral de Helmholtz no contorno combinada com equações adicionais compatíveis, formuladas a partir da equação integral de Helmholtz interna para alguns pontos internos, externos ao domínio infinito, localizados de forma conveniente.

Ainda na década de 60, SHAW (1968) utilizou a equação integral de contorno no problema da difusão de ondas elásticas em obstáculos rígidos. O RPT foi usado no caso de ondas elásticas, com as equações formuladas em termos de tensão e deformação na superfície ao invés de pressão e velocidade de onda normal à superfície. A diferença entre a onda elástica e acústica está no meio de propagação, elástico e fluido respectivamente.

Um método iterativo para resolver o problema da difusão acústica, chamado de método da matriz de transmissão, foi introduzido por WATERMAN (1969).

Outro avanço significativo no estudo da aplicação de métodos da equação integral em problemas governados pela equação de Laplace e de Helmholtz em domínios infinitos foi o realizado por BURTON \& MILLER (1971). Para resolver o problema da não unicidade de resposta apontado por Copley, os autores propõem que a unicidade seja recuperada derivando-se a equação integral segundo a direção normal ao contorno, utilizando-se em seguida uma combinação linear entre a equação primitiva e sua derivada. Esta combinação linear multiplicada por uma constante, gera uma equação com solução única para todas as freqüências, no caso de problemas de Neumann. O método, chamado de CONDOR (Composite Outward Normal Derivative Overlap Relation), gera núcleos de integração hiper-singulares.

Uma teoria completa para o problema de Dirichlet bidimensional descrito pela equação de Helmholtz no caso de contornos abertos foi formulada por HAYASHI (1973), onde analisou-se inclusive o comportamento dos pontos de extremidades.

O trabalho de Burton \& Miller foi estendido para o caso do problema de Dirichlet por KLEINMAN \& ROACH (1974), onde estudou-se os operadores integrais e as autofunções de forma mais aprofundada.

MEYER et al. (1978) desenvolveram um procedimento para o cálculo do campo acústico radiado em corpos tridimensionais a partir da representação integral da equação de Helmholtz. A técnica de solução do problema da não unicidade de resposta foi baseada no trabalho de Burton \& Miller, sendo denominada HGF (Helmholtz Gradient Formulation). Outro trabalho apresentado por MEYER et al. (1979) desenvolve um método analítico, válido para todos os números de ondas, para a determinação de campos acústicos produzidos 
pela radiação de superfícies simétricas axialmente com condições de contorno quaisquer. É importante notar que o número de ondas é diretamente proporcional à freqüência.

Uma técnica baseada no MEC para resolver problemas de não existência e não unicidade de resposta no caso da radiação acústica foi apresentada por PIASZCYK \& KLOSNER (1984). Utilizou-se uma função de impedância na superfície que serve de base para o cálculo do campo de pressão acústica próximo ao contorno. Como o sistema de equações resultante passa a ter mais equações que incógnitas é utilizado o procedimento dos mínimos quadrados para retornar o sistema à sua ordem original.

O trabalho de BROD (1984) formulou uma técnica numérica válida para todos os números de ondas em casos de problemas de radiação acústica. O problema da não unicidade foi resolvido pela expansão da função de Green para a equação de Helmholtz em uma série de funções ortogonais, obtendo-se um conjunto infinito de equações integrais de contorno. Estas equações possuem solução única para todos os números de ondas e suas soluções podem ser usadas para representar soluções no domínio infinito, na forma de séries algébricas.

SEYBERT et al. (1985) apresentaram uma formulação computacional para implementar a representação integral da equação de Helmholtz para problemas de radiação e difusão acústica associados com corpos tridimensionais no domínio da freqüência. Outro trabalho apresentado por SEYBERT (1986) et al. foi um modelo simplificado da mesma representação integral para corpos com simetria axial em forma e condições de contorno.

Um estudo aprofundado sobre a utilização e validade do CHIEF para resolver o problema da não unicidade de resposta foi conduzido por SEYBERT \& RENGARAJAN (1987). Os resultados da técnica CHIEF foram comparados com os da técnica HGF e estimou-se ainda o erro na resposta obtida ao se utilizar o CHIEF.

A formulação alternativa para a análise de autovalores em problemas governados pela equação de Helmholtz através do MEC foi introduzida por COSTA JUNIOR (1988). A formulação apresenta as soluções fundamentais da equação integral de contorno iguais às do problema potencial regido pela equação de Laplace, como conseqüência tem-se a influência de termos de domínio.

Outro método de elementos de contorno para resolver problemas de radiação acústica em domínios infinitos, chamado CHI (Coupled Helmholtz Integrals), foi proposto por CUNEFARE \& KOOPMAN (1989). Este método vale para qualquer número de ondas e o problema da não unicidade é resolvido pela técnica de combinação linear da equação de Helmholtz com sua derivada em relação à normal, proposta por Burton \& Miller. Utilizando a mesma técnica para resolver a não unicidade, AMINI et al. (1990) apresentaram um 
método baseado no MEC para a determinação do campo acústico que envolve estruturas finitas imersas em meio fluido homogêneo e infinito. O sistema linear obtido neste método é resolvido de forma iterativa.

YOON et al. (1990) utilizaram o MEC para resolver problemas de radiação e difusão acústica em casos de comprimentos de ondas bem menores que as dimensões do corpo imerso no fluido, menos que $5 \%$ da dimensão característica do corpo. Vale lembrar que fenômenos que geram comprimentos de ondas pequenos causam altas freqüências.

Para problemas tridimensionais de radiação acústica em meio submetido a fluxo uniforme subsônico, velocidade relacionada ao fluxo abaixo da velocidade do som, WU \& LEE (1994) formularam uma equação integral de contorno. Ao invés de utilizarem a equação integral de Helmholtz no domínio transformado (da freqüência), o equacionamento utiliza uma função de Green obtida a partir da equação diferencial do problema. A finalidade da função de Green é incorporar à equação integral de contorno a influência do fluxo na radiação acústica. LACERDA et al. (1996) realizaram formulação semelhante para casos bidimensionais.

A técnica CHIEF é a mais fácil de ser compreendida e implementada. Porém, é importante notar o interesse nos últimos anos a respeito da técnica CONDOR, comprovado recentemente pela publicação de YANG (1999). Este artigo é curioso, pois apresenta um método baseado na equação integral de contorno para resolver problemas de difusão acústica bidimensional, sem singularidades nas equações integrais.

WILTON (1978) publicou um artigo com a análise de estruturas finitas imersas em um meio acústico homogêneo infinito. Para resolver o problema do acoplamento fluidoestrutura, o autor utilizou o Método dos Elementos Finitos (MEF) para analisar a estrutura e uma formulação baseada na equação integral de contorno, apresentada por Schenck, para analisar o meio acústico. A estrutura pode ser uma fonte irradiadora de ondas acústicas ou apenas um obstáculo para ondas incidentes.

Outro artigo interessante, relacionado com a interação fluido-estrutura, é o publicado por BARRETTO et al. (1998). Nesse artigo é desenvolvida uma técnica de acoplamento fluido-estrutura (MEC-MEC) para o caso de pórticos planos. Os elementos do pórtico são acoplados através da técnica de sub-regiões utilizada no MEC.

O acoplamento fluido-estrutura pode ser feito de inúmeras maneiras; neste trabalho será utilizada uma formulação MEC-MEC. 


\subsection{Conceitos básicos}

O fenômeno da propagação de ondas acústicas em domínio bidimensional é um problema potencial, descrito pela equação de Helmholtz. Serão apresentadas as variáveis e hipóteses utilizadas na formulação da equação diferencial desenvolvida por Helmholtz.

\subsubsection{Equação de estado}

Inicia-se o desenvolvimento a partir da equação de estado, obtida através da equação de Poisson linearizada, que caracteriza o comportamento de um gás adiabáticon

$$
p=\beta \cdot \frac{\left(\rho-\rho_{0}\right)}{\rho_{0}}=\beta \cdot s
$$

Onde $p$ é a pressão acústica, diferença entre a pressão interna instantânea $P$ e a pressão de equilíbrio no fluido ${ }^{\square} P_{0}, \beta$ é o módulo volumétrico adiabático, constante determinada experimentalmente, $\rho$ é a densidade instantânea e $\rho_{0}$ a densidade de equilíbrio. Portanto, $s$ é a taxa de variação de densidade do fluido, também conhecida como condensação. A limitação para o uso de (1.1), de acordo com KINSLER et al. (1982), é que a condensação seja pequena, $|s|<<1$. Segundo KANE (1994), a equação $p=\beta \cdot s$ encontra analogia na teoria da elasticidade, através equação que relaciona tensão e deformação, lei de Hooke, $\sigma=E \cdot \varepsilon$.

\subsubsection{Equação de continuidade}

O próximo passo é encontrar uma relação entre velocidade de partícula $\vec{v}$ e $\rho$, chamada de equação de continuidade.

Considerando-se um fenômeno de transporte de massa em um elemento de volume infinitesimal $d_{V}$, na direção $X$ :

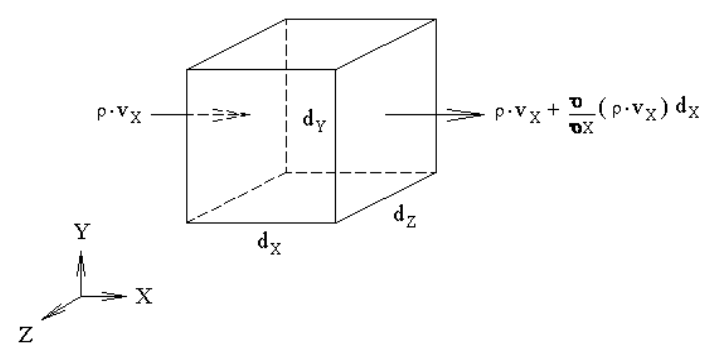

Figura 1.1 - Fluxo de massa na direção $X$ do volume infinitesimal

\footnotetext{
${ }^{1}$ Hipótese teórica, na qual não há trocas de energia térmica no fluido.

${ }^{2}$ Fluido significa substância com fraca adesão entre suas moléculas; sem forma característica.
} 
O fluxo de massa pode ser expresso pela seguinte relação:

$$
\left\{\rho \cdot v_{X}-\left[\rho \cdot v_{X}+\frac{\partial\left(\rho \cdot v_{X}\right)}{\partial X} d_{X}\right]\right\} \cdot d_{A}=-\frac{\partial\left(\rho \cdot v_{X}\right)}{\partial X} d_{V}
$$

Generalizando-se (1.2) para as direções $Y$ e $Z$ pode-se escrever o fluxo de massa através do operador divergente ${ }^{3}$.

$$
-\left[\frac{\partial\left(\rho \cdot v_{X}\right)}{\partial X}+\frac{\partial\left(\rho \cdot v_{Y}\right)}{\partial Y}+\frac{\partial\left(\rho \cdot v_{Z}\right)}{\partial Z}\right] \cdot d_{V}=-[\nabla \cdot(\rho \cdot \vec{v})] \cdot d_{V}
$$

A taxa de crescimento da massa em $d_{V}$, sinônimo de fluxo de massa, também pode ser expressa por $\frac{\partial \rho}{\partial t} d_{V}$.

$$
\begin{gathered}
\frac{\partial \rho}{\partial t} d_{V}=-[\nabla \cdot(\rho \cdot \vec{v})] \cdot d_{V} \\
\frac{\partial \rho}{\partial t}=-\nabla \cdot(\rho \cdot \vec{v}) \\
\frac{\partial \rho}{\partial t}+\nabla \cdot(\rho \cdot \vec{v})=0 \quad \text { (equação de continuidade) }
\end{gathered}
$$

A densidade instantânea pode ser expressa em função da condensação:

$$
\rho=\rho_{0} \cdot(1+s)
$$

A equação (1.4) pode ser linearizada. Considerando-se $s$ infinitesimal, $\rho \cong \rho_{0}$, e $\rho_{0}$ constante, tem-se:

$$
\begin{gathered}
\frac{\partial \rho}{\partial t}+\nabla \cdot\left(\rho_{0} \cdot \vec{v}\right)=0 \\
\frac{1}{\rho_{0}} \cdot \frac{\partial \rho}{\partial t}+\nabla \cdot(\vec{v})=0 \\
\frac{\partial\left(\frac{\rho}{\rho_{0}}\right)}{\partial t}+\nabla \cdot(\vec{v})=0 \\
\partial\left(\frac{\rho_{0} \cdot(1+s)}{\rho_{0}}\right) \\
\frac{\partial t}{t}+\nabla \cdot(\vec{v})=0
\end{gathered}
$$

${ }^{3}$ Operador divergente escrito em coordenadas cartesianas: $\nabla \cdot \vec{v}=\frac{\partial v_{X}}{\partial X}+\frac{\partial v_{Y}}{\partial Y}+\frac{\partial v_{Z}}{\partial Z}$ 


$$
\begin{gathered}
\frac{\partial(1+s)}{\partial t}+\nabla \cdot(\vec{v})=0 \\
\frac{\partial s}{\partial t}+\nabla \cdot(\vec{v})=0 \quad \text { (equação linearizada de continuidade) }
\end{gathered}
$$

Velocidade $(\vec{v})$ e pressão $(p)$ são condições de contorno usuais em problemas de acústica.

\subsubsection{Equação de Euler}

A equação de Euler relaciona pressão acústica $p$ com velocidade instantânea $\vec{v}$; é obtida através da consideração de um volume infinitesimal $d_{V}$ que se move com o fluido, com massa infinitesimal $d_{m}$. Considera-se um fluido adiabático e não viscoso, os efeitos da viscosidade $e^{4}$ no movimento são desprezados.

Pela Segunda lei de Newton obtém-se a expressão para força infinitesimal.

$$
d_{\vec{F}}=\vec{a} \cdot d_{m}
$$

Na direção $X$, a componente da força infinitesimal pode ser representada em termos da pressão interna instantânea $P$.

$$
d_{F x}=-\frac{\partial P}{\partial X} \cdot d_{V}
$$

Pode-se generalizar (1.8) para as direções $Y$ e $Z$, através do operador gradiente ${ }^{B}$.

$$
d_{\vec{F}}=-\frac{\partial P}{\partial X} \cdot d_{V} \cdot \mathbf{i}-\frac{\partial P}{\partial Y} \cdot d_{V} \cdot \mathbf{j}-\frac{\partial P}{\partial Z} \cdot d_{V} \cdot \mathbf{k}=-\nabla P d_{V}
$$

Uma partícula do fluido possui velocidade instantânea $\vec{v}(X, Y, Z, t)$ em uma posição $(X, Y, Z)$ em um determinado tempo $t$. Ao se deslocar para $\left(X+d_{X}, Y+d_{Y}, Z+d_{Z}\right)$ em um tempo $t+d_{t}$, a partícula adquiri uma nova velocidade $\vec{v}\left(X+d_{X}, Y+d_{Y}, Z+d_{Z}, t+d_{t}\right)$. Pela definição de aceleração, tem-se:

$$
\vec{a}=\frac{d_{\vec{v}}}{d_{t}}=\lim _{\Delta t \rightarrow 0}\left[\frac{\Delta \vec{v}}{\Delta t}\right]=\lim _{d_{t} \rightarrow 0}\left[\frac{\vec{v}\left(X+d_{X}, Y+d_{Y}, Z+d_{Z}, t+d_{t}\right)-\vec{v}(X, Y, Z, t)}{d_{t}}\right]
$$

Definindo-se velocidade como $\vec{v}=\frac{d_{\vec{u}}}{d_{t}}(\vec{u}=$ posição $)$, pode-se desenvolver (1.10):

\footnotetext{
${ }^{4}$ Grau de adesão entre as moléculas do fluido.

${ }^{5}$ Operador gradiente escrito em coordenadas cartesianas: $\nabla P=\frac{\partial P}{\partial X} \cdot \mathbf{i}+\frac{\partial P}{\partial Y} \cdot \mathbf{j}+\frac{\partial P}{\partial Z} \cdot \mathbf{k}$
} 


$$
\vec{a}=\lim _{d_{t} \rightarrow 0}\left[\frac{\vec{v}\left(X+v_{X} \cdot d_{t}, Y+v_{Y} \cdot d_{t}, Z+v_{Z} \cdot d_{t}, t+d_{t}\right)-\vec{v}(X, Y, Z, t)}{d_{t}}\right]
$$

Sendo infinitesimais os incrementos nas variáveis, a velocidade instantânea no tempo $t+d_{t}$ pode ser expressa pela expansão de Taylor até o termo de derivada primeira.

$$
\begin{aligned}
\vec{v}\left(X+v_{X} \cdot d_{t}, Y+v_{Y} \cdot d_{t}, Z+v_{Z} \cdot d_{t}, t+d_{t}\right)= & \vec{v}(X, Y, Z, t)+\frac{\partial \vec{v}}{\partial X} \cdot v_{X} d_{t}+\frac{\partial \vec{v}}{\partial Y} \cdot v_{Y} d_{t} \\
& +\frac{\partial \vec{v}}{\partial Z} \cdot v_{Z} d_{t}+\frac{\partial \vec{v}}{\partial t} d_{t}
\end{aligned}
$$

Portanto, a expressão (1.11) adquiri a forma:

$$
\begin{gathered}
\vec{a}=\lim _{d_{t} \rightarrow 0}\left[\frac{\vec{v}(X, Y, Z, t)+\frac{\partial \vec{v}}{\partial X} \cdot v_{X} d_{t}+\frac{\partial \vec{v}}{\partial Y} \cdot v_{Y} d_{t}+\frac{\partial \vec{v}}{\partial Z} \cdot v_{Z} d_{t}+\frac{\partial \vec{v}}{\partial t} d_{t}-\vec{v}(X, Y, Z, t)}{d_{t}}\right] \\
=\lim _{d_{t} \rightarrow 0}\left[\frac{\frac{\partial \vec{v}}{\partial X} \cdot v_{X} d_{t}+\frac{\partial \vec{v}}{\partial Y} \cdot v_{Y} d_{t}+\frac{\partial \vec{v}}{\partial Z} \cdot v_{Z} d_{t}+\frac{\partial \vec{v}}{\partial t} d_{t}}{d_{t}}\right] \\
=\lim _{d_{t} \rightarrow 0}\left[\frac{\partial \vec{v}}{\partial X} \cdot v_{X}+\frac{\partial \vec{v}}{\partial Y} \cdot v_{Y}+\frac{\partial \vec{v}}{\partial Z} \cdot v_{Z}+\frac{\partial \vec{v}}{\partial t}\right] \\
\vec{a}=\frac{\partial \vec{v}}{\partial t}+v_{X} \cdot \frac{\partial \vec{v}}{\partial X}+v_{Y} \cdot \frac{\partial \vec{v}}{\partial Y}+v_{Z} \cdot \frac{\partial \vec{v}}{\partial Z}
\end{gathered}
$$

Se o operador vetorial $(\vec{v} \cdot \nabla)$ for definido como:

$$
(\vec{v} \cdot \nabla)=v_{X} \cdot \frac{\partial}{\partial X}+v_{Y} \cdot \frac{\partial}{\partial Y}+v_{Z} \cdot \frac{\partial}{\partial Z}
$$

Tem-se:

$$
\vec{a}=\frac{\partial \vec{v}}{\partial t}+(\vec{v} \cdot \nabla)(\vec{v})
$$

A massa infinitesimal pode ser escrita da seguinte maneira:

$$
d_{m}=\rho \cdot d_{V}
$$

Substituindo-se as equações (1.9), (1.15) e (1.16) em (1.7), chega-se a uma nova equação.

$$
-\nabla P d_{V}=\left[\frac{\partial \vec{v}}{\partial t}+(\vec{v} \cdot \nabla)(\vec{v})\right] \cdot \rho d_{V}
$$

$-\nabla P=\left[\frac{\partial \vec{v}}{\partial t}+(\vec{v} \cdot \nabla)(\vec{v})\right] \cdot \rho \quad$ (equação de Euler para fluidos não viscosos) 
A consideração de condensação infinitesimal implica em $\rho \cong \rho_{0}$. Se a pressão de equilíbrio no fluido $\left(P_{0}\right)$ for constante, $\nabla P=\nabla p$. Lembrando-se que $p=P-P_{0}$. Outra simplificação possível é a consideração de $|(\vec{v} \cdot \nabla)(\vec{v})|<<\left|\frac{\partial \vec{v}}{\partial t}\right|$. Através destas hipóteses é possível obter-se a equação (1.17) na forma linearizada, válida para fenômenos acústicos de pequena amplitude.

$-\nabla p=\rho_{0} \cdot \frac{\partial \vec{v}}{\partial t} \quad$ (equação linear de Euler para fluidos não viscosos)

As equações de continuidade e de Euler foram baseadas em KINSLER et al. (1982).

\subsubsection{Equação transiente de ondas}

Aplicando-se o operador divergente na equação (1.18), obtém-se:

$$
\begin{gathered}
\rho_{0} \cdot \nabla \cdot\left(\frac{\partial \vec{v}}{\partial t}\right)=-\nabla \cdot(\nabla p)=-\nabla^{2} p \\
\nabla \cdot\left(\frac{\partial \vec{v}}{\partial t}\right)=-\frac{\nabla^{2} p}{\rho_{0}}
\end{gathered}
$$

Na equação acima, $\nabla^{2}$ é o operador Laplaciano .

Derivando-se a equação (1.6) em relação ao tempo:

$$
\frac{\partial^{2} s}{\partial t^{2}}+\nabla \cdot \frac{\partial(\vec{v})}{\partial t}=0
$$

As equações (1.19) e (1.20) podem ser combinadas numa equação única.

$$
\frac{\partial^{2} s}{\partial t^{2}}-\frac{\nabla^{2} p}{\rho_{0}}=0
$$

Da equação (1.1) tira-se a relação $s=\frac{p}{\beta}$, que pode ser substituída em (1.21).

$$
\begin{aligned}
& \frac{1}{\beta} \cdot \frac{\partial^{2} p}{\partial t^{2}}-\frac{\nabla^{2} p}{\rho_{0}}=0 \\
& \frac{\rho_{0}}{\beta} \cdot \frac{\partial^{2} p}{\partial t^{2}}-\nabla^{2} p=0
\end{aligned}
$$

${ }^{6}$ Operador Laplaciano escrito em coordenadas cartesianas: $\nabla^{2} p=\frac{\partial^{2} p}{\partial X^{2}}+\frac{\partial^{2} p}{\partial Y^{2}}+\frac{\partial^{2} p}{\partial Z^{2}}$ 


$$
\nabla^{2} p=\frac{1}{c^{2}} \cdot \frac{\partial^{2} p}{\partial t^{2}}
$$

(equação de onda linearizada, expressa em termos de pressão acústica)

Onde a constante $c=\sqrt{\frac{\beta}{\rho_{0}}}$ é chamada de velocidade de propagação (ou fase) da onda acústica.

Definindo-se velocidade instantânea como um gradiente de uma função escalar $\phi$, denominada potencial de velocidade de onda, escreve-se $\vec{v}=\nabla \phi$. Portanto a equação (1.18) pode ser escrita como:

$$
\begin{gathered}
-\nabla p=\rho_{0} \cdot \frac{\partial(\nabla \phi)}{\partial t} \\
\nabla\left(\rho_{0} \cdot \frac{\partial \phi}{\partial t}+p\right)=0
\end{gathered}
$$

Segundo KINSLER et al. (1982), caso não haja excitação acústica, a expressão entre parênteses na equação (1.23) é igual a zero.

$$
\begin{gathered}
\rho_{0} \cdot \frac{\partial \phi}{\partial t}+p=0 \\
p=-\rho_{0} \cdot \frac{\partial \phi}{\partial t}
\end{gathered}
$$

Substituindo-se (1.24) na equação (1.22), prova-se que a mesma equação linearizada pode ser utilizada em termos de potencial de velocidade de onda acústica.

$$
\begin{aligned}
\nabla^{2}\left(-\rho_{0} \cdot \frac{\partial \phi}{\partial t}\right) & =\frac{1}{c^{2}} \cdot \frac{\partial^{2}\left(-\rho_{0} \cdot \frac{\partial \phi}{\partial t}\right)}{\partial t^{2}} \\
-\rho_{0} \cdot \frac{\partial\left(\nabla^{2} \phi\right)}{\partial t} & =-\rho_{0} \cdot \frac{1}{c^{2}} \cdot \frac{\partial}{\partial t} \frac{\partial}{\partial t}\left(\frac{\partial \phi}{\partial t}\right) \\
\nabla^{2} \phi & =\frac{1}{c^{2}} \cdot \frac{\partial}{\partial t}\left(\frac{\partial \phi}{\partial t}\right) \\
\nabla^{2} \phi & =\frac{1}{c^{2}} \cdot \frac{\partial^{2} \phi}{\partial t^{2}}
\end{aligned}
$$

(equação de onda linearizada, expressa em termos de potencial de velocidade de onda acústica) 


\subsubsection{Equação harmônica de ondas (Equação de Helmholtz)}

As equações (1.22) e (1.25) estão expressas no domínio do tempo, são equações transientes de propagação de ondas acústicas. O objetivo desta dissertação é trabalhar com problemas no domínio da freqüência; portanto, é necessário obter uma equação que atenda a esse requisito.

Se for considerado que as ondas sejam produzidas por vibrações periódicas no tempo, harmônicas, com resposta também periódica por parte do domínio de propagação, fluido, pode-se escrever a pressão acústica na forma de uma função com comportamento de série.

$$
p(u, t)=p(u) \cdot e^{-i \cdot \omega \cdot t}
$$

Na equação acima, $\omega$ representa a freqüência angular da vibração e resposta, ambas com comportamento harmônico; $i$ é a representação da parte imaginária de um número complexo. Substituindo-se (1.26) na equação (1.22), tem-se:

$$
\begin{gathered}
\nabla^{2}\left(p \cdot e^{-i \cdot \omega \cdot t}\right)=\frac{1}{c^{2}} \cdot \frac{\partial^{2}\left(p \cdot e^{-i \cdot \omega \cdot t}\right)}{\partial t^{2}} \\
e^{-i \cdot \omega \cdot t} \cdot \nabla^{2} p=\frac{1}{c^{2}} \cdot \frac{\partial\left(p \cdot e^{-i \cdot \omega \cdot t} \cdot-i \cdot \omega\right)}{\partial t} \\
e^{-i \cdot \omega \cdot t} \cdot \nabla^{2} p=\frac{1}{c^{2}} \cdot p \cdot e^{-i \cdot \omega \cdot t} \cdot(-i \cdot \omega)^{2} \\
\nabla^{2} p=-\frac{\omega^{2}}{c^{2}} \cdot p \\
\nabla^{2} p+\left(\frac{\omega}{c}\right)^{2} \cdot p=0 \\
\nabla^{2} p+K^{2} \cdot p=0
\end{gathered}
$$

(equação diferencial de Helmholtz, expressa em termos de pressão acústica)

A constante $K=\frac{\omega}{c}$ é chamada de número de ondas; quando $\omega$ é expresso em radianos, $K$ indica o número de ondas compreendidas em uma distância de $2 \pi$ unidades.

Analogamente, a partir da equação (1.25) a equação de Helmholtz pode ser expressa em termos de potencial de velocidade de onda.

$$
\nabla^{2} \phi+K^{2} \cdot \phi=0
$$

(equação diferencial de Helmholtz, expressa em termos de potencial de velocidade de onda acústica) 


\section{O MÉTODO DOS ELEMENTOS DE CONTORNO APLICADO A PROBLEMAS BIDIMENSIONAIS GOVERNADOS PELA EQUAÇÃO DE HELMHOLTZ}

\subsection{Núcleos de pressão acústica}

No item 1.2 do capítulo 1, foram desenvolvidas equações diferenciais homogêneas para o fenômeno da propagação de ondas em meio fluido. Segundo KINSLER et al. (1982), também é possível a obtenção de equações semelhantes para o caso de fontes de energia acústica presentes no meio. $\mathrm{O}$ autor apresenta duas situações onde existem termos de domínio geradores de energia acústica:

(a) Quando há uma taxa de variação de massa no domínio $G(u, t)$, massa por unidade de volume. Com base na equação linearizada de continuidade (1.6), chega-se à seguinte equação:

$$
\rho_{0} \cdot \frac{\partial s}{\partial t}+\rho_{0} \cdot \nabla \cdot(\vec{v})=G
$$

A densidade de equilíbrio $\rho_{0}$ é introduzida para ajustar a equação dimensionalmente.

(b) Quando existem forças de corpo no fluido. Partindo-se da equação linear de Euler (1.18), com a introdução de um termo $\vec{F}(u, t)$ que representa uma força de corpo por unidade de volume, chega-se à uma nova equação.

$$
\rho_{0} \cdot \frac{\partial \vec{v}}{\partial t}+\nabla p=\vec{F}
$$

As equações (2.1) e (2.2) combinadas com a equação de estado (1.1) geram uma equação de onda linearizada diferencial não homogênea, expressa em termos de pressão acústica, que contempla as duas formas apresentadas de fontes de energia acústica. 


$$
\nabla^{2} p-\frac{1}{c^{2}} \cdot \frac{\partial^{2} p}{\partial t^{2}}=-\frac{\partial G}{\partial t}+\nabla \cdot \vec{F}
$$

Não é o escopo deste trabalho o estudo da natureza da fonte de energia acústica. Para efeitos práticos, os termos relacionados à essas fontes, representados por $D$, serão considerados constantes no espaço e de comportamento harmônico no tempo. De forma genérica, esses termos de domínio serão denominados núcleos de pressão acústica.

$$
\nabla^{2} p-\frac{1}{c^{2}} \cdot \frac{\partial^{2} p}{\partial t^{2}}=D
$$

Com base nas considerações apresentadas, a equação acima pode ser desenvolvida numa equação diferencial não homogênea, de maneira semelhante à apresentada no desenvolvimento da equação (1.27). Com $D(u, t)=D(u) \cdot e^{-i \cdot \omega \cdot t}$.

$$
\nabla^{2} p+K^{2} \cdot p=D \quad \text { (equação diferencial de Helmholtz não homogênea) }
$$

\subsection{Equação integral de contorno}

\subsubsection{Equacionamento básico}

O conceito inicial para o desenvolvimento da equação integral de contorno é o de fluxo, representado por $q$. O sentido da palavra fluxo no problema harmônico analisado pelo MEC é o de potencial de pressão $p$ que passa por uma seção transversal $A$, tomada segundo a direção normal $\eta$. Portanto, a condição de rigidez acústica, não propagação de ondas através da seção, é de $q=0$.

$$
q=\frac{\partial p}{\partial \eta} \quad \text { (fluxo de pressão acústica) }
$$

Neste trabalho serão estudados os domínios: finito (representado por $\Omega$ ) e infinito (representado por $\Omega_{\infty}$ ). Para os dois casos o contorno é expresso por $\Gamma$.

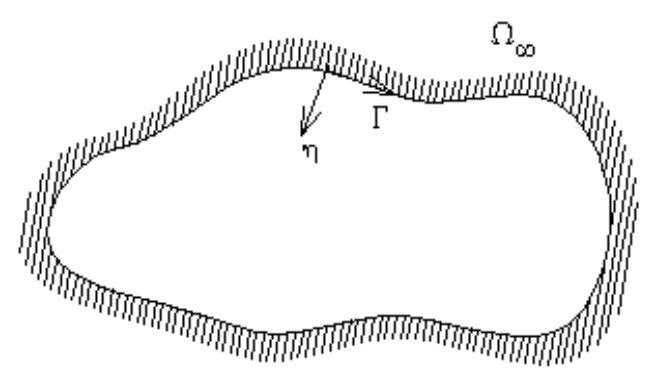

Domínio infinito

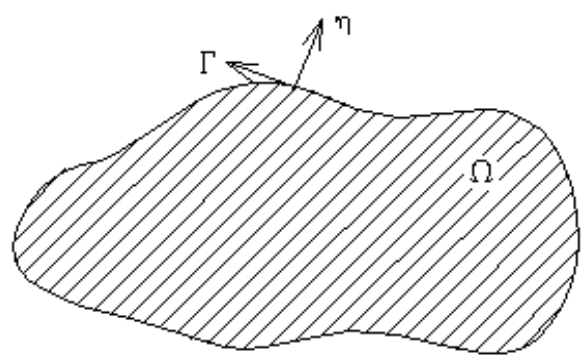

Domínio finito

Figura 2.1 - Tipos de domínios 
Para resolver a equação diferencial de Helmholtz (1.27) nos dois tipos de domínio, podem ser utilizadas três condições de contorno:

(a) Condição de Dirichlet $(p=\bar{p})$ : potencial de pressão acústica prescrito e fluxo incógnito.

(b) Condição de Neumann $(q=\bar{q})$ : fluxo de pressão acústica prescrito e potencial incógnito.

(c) Condição de Robin $\left(f_{1} \cdot p+f_{2} \cdot q=f_{3}\right)$ : condição mista entre (a) e (b). $f_{1}, f_{2}$ e $f_{3}$ são funções conhecidas.

No caso de domínio infinito, tem-se ainda uma quarta condição de contorno, conhecida como de Sommerfeld, de acordo com CISKOWSKI \& BREBBIA (1991).

(d) Condição radiação de Sommerfeld no infinito: $q=i \cdot K \cdot p$

Pode-se relacionar a equação diferencial não homogênea do problema (2.5) com uma função de erro $\varepsilon_{(p)}$.

$$
\nabla^{2} p+K^{2} \cdot p-D=0 \cong \varepsilon_{(p)}
$$

Utilizando-se uma função ponderadora $p^{*}$, calcula-se o erro ponderado no domínio através de um procedimento de resíduos ponderados:

$$
\int_{\Omega}\left(\nabla^{2} p+K^{2} \cdot p-D\right) \cdot p^{*} d_{\Omega}=0
$$

Através da identidade vetorial $\nabla \cdot\left(p^{*} \nabla p\right)=\nabla p^{*} \cdot \nabla p+p^{*} \nabla^{2} p$ extraída do teorema de Green chega-se à expressão $p^{*} \nabla^{2} p=\nabla \cdot\left(p^{*} \nabla p\right)-\nabla p^{*} \cdot \nabla p$, que substituída na equação (2.8) fornece:

$$
\int_{\Omega}\left[\nabla \cdot\left(p^{*} \nabla p\right)-\nabla p^{*} \cdot \nabla p+K^{2} \cdot p \cdot p^{*}-D \cdot p^{*}\right] d_{\Omega}=0
$$

Aplicando-se o teorema da divergência ${ }^{2}$ na equação acima, tem-se:

$$
\int_{\Gamma}\left(p^{*} \nabla p\right) \cdot \eta d_{\Gamma}+\int_{\Omega}\left(-\nabla p^{*} \cdot \nabla p+K^{2} \cdot p \cdot p^{*}-D \cdot p^{*}\right) d_{\Omega}=0
$$

Sabe-se que $\nabla p \cdot \eta=\nabla p$, pois: $\left(\frac{\partial p}{\partial X_{i}} \cdot \eta_{i}\right) \cdot \eta_{i}=\frac{\partial p}{\partial X_{i}} \cdot \eta_{i} \cdot \delta_{i i}=\frac{\partial p}{\partial X_{i}} \cdot \eta_{i}=\nabla p$.

Logo, a equação (2.10) pode ser escrita da seguinte maneira:

\footnotetext{
${ }^{1}$ Teorema de Green: $\int_{\Omega}\left(p^{*} \nabla^{2} p-p \nabla^{2} p^{*}\right) d_{\Omega}=\int_{\Gamma}\left(p^{*} \nabla p-p \nabla p^{*}\right) \cdot \eta d_{\Gamma}$

${ }^{2}$ Teorema da divergência: $\int_{\Omega} \nabla \cdot \vec{F} d_{\Omega}=\int_{\Gamma} \vec{F} \cdot \eta d_{\Gamma}$
} 


$$
\int_{\Gamma} p^{*} \nabla p d_{\Gamma}+\int_{\Omega}\left(-\nabla p^{*} \cdot \nabla p+K^{2} \cdot p \cdot p^{*}-D \cdot p^{*}\right) d_{\Omega}=0
$$

Aplicando-se a regra da cadeia na definição de fluxo:

$$
\frac{\partial p}{\partial \eta}=\frac{\partial p}{\partial X_{i}} \cdot \frac{\partial X_{i}}{\partial \eta_{i}}=\frac{\partial p}{\partial X_{i}} \cdot \eta_{i}=\nabla p
$$

Substituindo-se (2.12) em (2.11):

$$
\int_{\Gamma} p^{*} \cdot \frac{\partial p}{\partial \eta} d_{\Gamma}+\int_{\Omega}\left(-\nabla p^{*} \cdot \nabla p+K^{2} \cdot p \cdot p^{*}-D \cdot p^{*}\right) d_{\Omega}=0
$$

Uma Segunda identidade vetorial pode ser obtida, a partir do teorema de Green: $\nabla \cdot\left(p \nabla p^{*}\right)=\nabla p \cdot \nabla p^{*}+p \nabla^{2} p^{*}$, que reordenada adquiri a seguinte configuração: $\nabla p \cdot \nabla p^{*}=\nabla p^{*} \cdot \nabla p=\nabla \cdot\left(p \nabla p^{*}\right)-p \nabla^{2} p^{*}$, podendo ser então substituída em (2.13).

$$
\int_{\Gamma} p^{*} \cdot \frac{\partial p}{\partial \eta} d_{\Gamma}+\int_{\Omega}-\left[\nabla \cdot\left(p \nabla p^{*}\right)-p \nabla^{2} p^{*}\right] d_{\Omega}+\int_{\Omega}\left(K^{2} \cdot p \cdot p^{*}-D \cdot p^{*}\right) d_{\Omega}=0
$$

Aplicando-se novamente o teorema da divergência no termo $\int_{\Omega} \nabla \cdot\left(p \nabla p^{*}\right) d_{\Omega}$, podese desenvolver a equação (2.14).

$$
\begin{gathered}
\int_{\Omega} \nabla \cdot\left(p \nabla p^{*}\right) d_{\Omega}=\int_{\Gamma}\left(p \nabla p^{*}\right) \cdot \eta d_{\Gamma}=\int_{\Gamma} p \cdot \frac{\partial p^{*}}{\partial \eta} d_{\Gamma} \\
\int_{\Gamma} p^{*} \cdot \frac{\partial p}{\partial \eta} d_{\Gamma}-\int_{\Gamma} p \cdot \frac{\partial p^{*}}{\partial \eta} d_{\Gamma}+\int_{\Omega}\left(p \nabla^{2} p^{*}+K^{2} \cdot p \cdot p^{*}-D \cdot p^{*}\right) d_{\Omega}=0 \\
\int_{\Gamma} p^{*} \cdot \frac{\partial p}{\partial \eta} d_{\Gamma}-\int_{\Gamma} p \cdot \frac{\partial p^{*}}{\partial \eta} d_{\Gamma}+\int_{\Omega}\left(\nabla^{2} p^{*}+K^{2} \cdot p^{*}\right) \cdot p d_{\Omega}-\int_{\Omega} D \cdot p^{*} d_{\Omega}=0
\end{gathered}
$$

\subsubsection{Solução fundamental}

Para continuar o desenvolvimento da equação integral de contorno é necessário a introdução dos conceitos da função delta de Dirac $\delta_{(S, P)}$, e solução fundamental.

A função $\delta_{(S, P)}$ representa a aplicação de quantidades (cargas, potenciais, etc.) unitárias concentradas em um ponto $S$, chamado de ponto de colocação ou ponto fonte. $P$ é um ponto do domínio, onde se realiza a integração. A definição, em linguagem algébrica, de $\delta_{(S, P)}$ pode ser feita da seguinte maneira:

$$
\delta_{(S, P)}=\begin{array}{ll}
\infty & \text { em } S=P \\
0 & \text { em } S \neq P
\end{array} \Rightarrow \int_{\Omega} \delta_{(S, P)} d_{\Omega}=1
$$


Como conseqüência da definição apresentada em (2.16), tem-se a seguinte propriedade de $\delta_{(S, P)}$ :

$$
\int_{\Omega} \delta_{(S, P)} \cdot p_{(P)} d_{\Omega}=p_{(S)}
$$

Caso a função ponderadora $p^{*}$ utilizada em (2.8) satisfaça a equação diferencial de Helmholtz (1.27), que governa o problema, e represente a solução para o potencial de pressão no ponto de colocação $S$, no problema de domínio infinito com uma fonte unitária aplicada no ponto $P$, então $p^{*}$ é a solução fundamental.

$$
\begin{gathered}
\nabla^{2} p^{*}+K^{2} \cdot p^{*}+\delta_{(S, P)}=0 \\
\left(\nabla^{2} p^{*}+K^{2} \cdot p^{*}\right) \cdot p=-\delta_{(S, P)} \cdot p_{(P)} \\
\int_{\Omega}\left(\nabla^{2} p^{*}+K^{2} \cdot p^{*}\right) \cdot p d_{\Omega}=\int_{\Omega}-\delta_{(S, P)} \cdot p_{(P)} d_{\Omega} \\
\int_{\Omega}\left(\nabla^{2} p^{*}+K^{2} \cdot p^{*}\right) \cdot p d_{\Omega}=-p_{(S)}
\end{gathered}
$$

Substituindo-se (2.18) em (2.15) chega-se à equação integral de contorno.

$$
\int_{\Gamma} p^{*} \cdot \frac{\partial p}{\partial \eta} d_{\Gamma}-\int_{\Gamma} p \cdot \frac{\partial p^{*}}{\partial \eta} d_{\Gamma}-p_{(S)}-\int_{\Omega} D \cdot p^{*} d_{\Omega}=0
$$

A equação acima foi obtida para pontos de colocação $S$ pertencentes ao domínio. Uma maneira mais geral de representá-la, na qual $S$ pode estar localizado no domínio, no contorno ou fora do domínio, pode ser formulada utilizando-se um termo livre $c_{(S)}$ relacionado à posição de $S$. Os valores de $c_{(S)}$ são clássicos no MEC e podem ser encontrados em BREBBIA \& DOMINGUEZ (1992).

$$
c_{(S)} \cdot p_{(s)}+\int_{\Gamma} \frac{\partial p^{*}}{\partial \eta} \cdot p d_{\Gamma}=\int_{\Gamma} p^{*} \cdot \frac{\partial p}{\partial \eta} d_{\Gamma}-\int_{\Omega} D \cdot p^{*} d_{\Omega}
$$

(equação integral de contorno)

Onde:

$c_{(S)}=0$ para pontos $S$ externos ao domínio.

$c_{(S)}=\frac{\alpha}{2 \pi}$ para pontos $S$ no contorno ( $\alpha$ é o ângulo no contorno, para contorno suaves: $\left.c_{(S)}=\frac{\pi}{2 \pi}=0.5\right)$.

$c_{(S)}=1$ para pontos $S$ internos ao domínio. 
A solução fundamental $p^{*}$ e sua derivada em relação à reta normal $q^{*}=\frac{\partial p^{*}}{\partial \eta}$ podem ser encontradas em CISKOWSKI \& BREBBIA (1991).

$$
\begin{gathered}
p^{*}=\frac{i}{4} \cdot H_{0}^{(1)}(K R)=\frac{1}{4} \cdot\left[i \cdot J_{0}(K R)-Y_{0}(K R)\right] \\
q^{*}=\frac{\partial p^{*}}{\partial \eta}=-\frac{i \cdot K}{4} \cdot H_{1}^{(1)}(K R) \cdot \frac{\partial R}{\partial \eta}=-\frac{K}{4} \cdot\left[i \cdot J_{1}(K R)-Y_{1}(K R)\right] \cdot \frac{\partial R}{\partial \eta}
\end{gathered}
$$

$J_{n}$ e $Y_{n}$ são funções de Bessel do primeiro e segundo tipo, respectivamente. $Y_{n}$ também é chamada de função de Neumann. $H_{n}^{(1)}$ é uma função de Bessel do terceiro tipo de $1^{\text {a }}$ classe, também conhecida como função de Hankel. A variável $R$ indica a distância entre o ponto de colocação $S$ e o ponto de integração $P$. O índice $n$ representa a ordem da função.

\subsection{Representação matricial do MEC}

\subsubsection{Generalidades}

Conforme exposto na introdução, o MEC é um método numérico que trabalha com variáveis relacionadas a elementos discretos no contorno. Os chamados elementos de contorno definem a forma do domínio; o sentido em que é feita a integração define se o domínio é finito (sentido anti-horário) ou infinito (sentido horário). Os elementos de contorno, no caso bidimensional, podem ser retas ou curvas, de dimensões finitas, conectadas entre si. Estão relacionadas a estes elementos, as distribuições das variáveis de contorno, no caso de problemas potenciais: potencial e fluxo. As distribuições das variáveis de contorno assumem formas de funções polinomiais: constante, linear, quadrática, etc.; para cada tipo de distribuição estão associadas funções de forma $\phi$. Neste trabalho serão utilizadas distribuições constantes e lineares para as variáveis de contorno; o elemento de contorno adotado é o linear.

(a) Distribuição constante: um nó central no elemento, $\phi_{1}=1$.

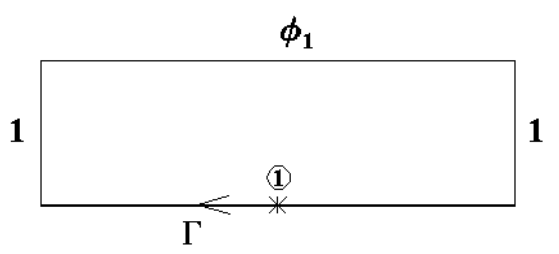

Figura 2.2 - Distribuição constante das variáveis de contorno 
(b) Distribuição linear: dois nós de extremidade no elemento, $\phi_{1}=\frac{1}{2} \cdot(1-\xi)$ e $\phi_{2}=\frac{1}{2} \cdot(1+\xi)$


Figura 2.3 - Distribuição linear da variável potencial de pressão acústica no contorno

A distribuição do fluxo de pressão acústica assume distribuição semelhante à apresentada na figura acima.

Os potenciais e fluxos nos nós de extremidade comuns a dois elementos adjacentes serão considerados no equacionamento como variáveis independentes, gerando o chamado nó descontínuo em potencial e fluxo. Para fluxo pode existir descontinuidade, pois as retas $\eta$ dos elementos lineares adjacentes podem ter orientações diferentes. No entanto, o potencial, por ser um valor escalar, é contínuo. Optou-se pela utilização de potenciais descontínuos pela facilidade de implementação computacional e porque as diferenças dos potenciais em nós adjacentes indicam a precisão dos resultados, quanto menores as diferenças, menores os erros do modelo implementado.

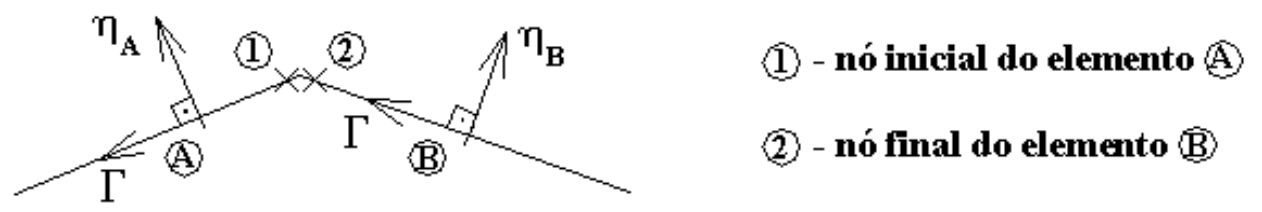

Figura 2.4 - Exemplo de nó descontínuo

\subsubsection{Sistema de equações algébricas}

Discretizando-se o contorno do domínio em $N E$ elementos é possível desenvolver um sistema algébrico de equações. Inicialmente consideram-se distribuições constantes de 
potencial e fluxo. O sistema é obtido para pontos de colocação relacionados com os $N N D$ nós pertencentes aos elementos, no caso um nó central por elemento. Para cada ponto de colocação $S$ é feita a integração de todos os elementos de contorno, gerando-se $N E$ equações que são linearmente independentes $\frac{\text { 目 }}{\text {. }}$
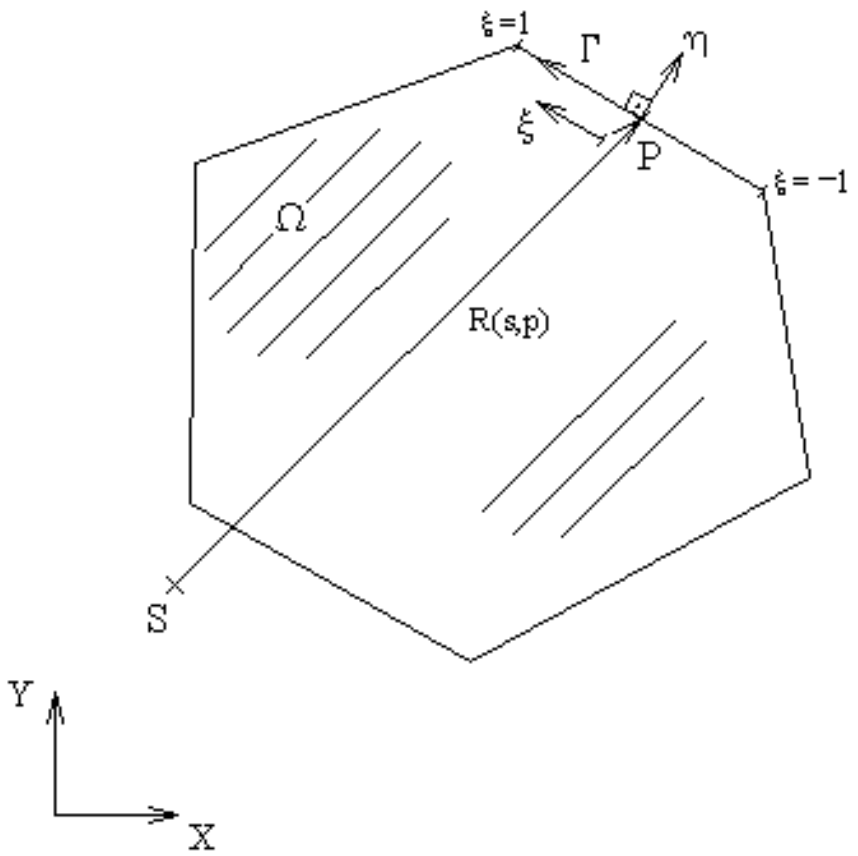

Figura 2.5 - Corpo discretizado em elementos lineares de contorno

A partir da equação (2.20) pode-se escrever um sistema algébrico com as $N E$ equações representadas por um índice $i$.

$$
c_{(S)} \cdot p_{i}+\int_{\Gamma} \frac{\partial p^{*}}{\partial \eta} \cdot p d_{\Gamma}=\int_{\Gamma} p^{*} \cdot q d_{\Gamma}-D_{i}
$$

O termo de domínio $D_{i}=\int_{\Omega} D \cdot p^{*} d_{\Omega}$ é integrado em relação a cada ponto $S$, gerando uma contribuição em cada equação.

Metade das variáveis é prescrita, conhecida, e a outra metade incógnita. Portanto, cada equação apresentará apenas $N E$ incógnitas, representadas por um índice $j$. Cada variável, prescrita ou incógnita, está multiplicada por um coeficiente, obtido a partir da integração das soluções fundamentais no elemento de contorno a que a variável pertence.

$$
c_{(S)} \cdot p_{i}+\sum_{j=1}^{N E}\left(\int_{\Gamma} \frac{\partial p^{*}}{\partial \eta} d_{\Gamma}\right) \cdot p_{j}=\sum_{j=1}^{N E}\left(\int_{\Gamma} p^{*} d_{\Gamma}\right) \cdot q_{j}-D_{i}
$$

\footnotetext{
${ }^{3}$ Equações que não são combinações lineares umas das outras.
} 


$$
\begin{gathered}
c_{(S)} \cdot p_{i}+\sum_{j=1}^{N E} \hat{H}_{i j} \cdot p_{j}=\sum_{j=1}^{N E} G_{i j} \cdot q_{j}-D_{i} \\
\sum_{j=1}^{N E} H_{i j} \cdot p_{j}=\sum_{j=1}^{N E} G_{i j} \cdot q_{j}-D_{i}
\end{gathered}
$$

Onde:

$$
H_{i j}=\begin{array}{cc}
\hat{H}_{i j} & \text { para } i \neq j \\
\hat{H}_{i j}+c_{(s)} & \text { para } i=j
\end{array}
$$

Os valores de $c_{(S)}$ podem ser encontrados no item 2.2.2 (solução fundamental).

A representação matricial da equação (2.22) fica:

$$
[H] \cdot\{p\}=[G] \cdot\{q\}-\{D\}
$$

Para a obtenção do sistema final de equações, pode-se considerar o exemplo abaixo:

$$
\{p\}=\left\{\begin{array}{c}
p_{1} \\
p_{2} \\
\vdots \\
\bar{p}_{j} \\
\vdots \\
p_{N}
\end{array}\right\} \quad \text { e }\{q\}=\left\{\begin{array}{c}
\bar{q}_{1} \\
\bar{q}_{2} \\
\vdots \\
q_{j} \\
\vdots \\
\bar{q}_{N}
\end{array}\right\}
$$

Onde:

$N=N E$ para o caso de distribuições constantes.

$\bar{p}_{j}, \bar{q}_{1}, \bar{q}_{2}$ e $\bar{q}_{N}$ são valores prescritos.

$p_{1}, p_{2}, p_{N}$ e $q_{j}$ são valores incógnitos.

De forma que o sistema matricial (2.24) fica representado por:

$$
\begin{aligned}
& {\left[\begin{array}{cccccc}
H_{11} & H_{12} & \ldots & H_{1 j} & \ldots & H_{1 N} \\
H_{21} & H_{22} & \ldots & H_{2 j} & \ldots & H_{2 N} \\
\vdots & \vdots & & \vdots & & \vdots \\
H_{N 1} & H_{N 2} & \ldots & H_{N j} & \ldots & H_{N N}
\end{array}\right] \cdot\left\{\begin{array}{c}
p_{1} \\
p_{2} \\
\vdots \\
\bar{p}_{j} \\
\vdots \\
p_{N}
\end{array}\right\}=} \\
& {\left[\begin{array}{cccccc}
G_{11} & G_{12} & \ldots & G_{1 j} & \ldots & G_{1 N} \\
G_{21} & G_{22} & \ldots & G_{2 j} & \ldots & G_{2 N} \\
\vdots & \vdots & & \vdots & & \vdots \\
G_{N 1} & G_{N 2} & \ldots & G_{N j} & \ldots & G_{N N}
\end{array}\right] \cdot\left\{\begin{array}{c}
\bar{q}_{1} \\
\bar{q}_{2} \\
\vdots \\
q_{j} \\
\vdots \\
\bar{q}_{N}
\end{array}\right\}-\left\{\begin{array}{c}
D_{1} \\
D_{2} \\
\vdots \\
D_{j} \\
\vdots \\
D_{N}
\end{array}\right\}}
\end{aligned}
$$


Pode-se isolar todos os valores prescritos de um mesmo lado da equação:

$$
\begin{aligned}
& {\left[\begin{array}{cccccc}
H_{11} & H_{12} & \ldots & -G_{1 j} & \ldots & H_{1 N} \\
H_{21} & H_{22} & \ldots & -G_{2 j} & \ldots & H_{2 N} \\
\vdots & \vdots & & \vdots & & \vdots \\
H_{N 1} & H_{N 2} & \ldots & -G_{N j} & \ldots & H_{N N}
\end{array}\right] \cdot\left\{\begin{array}{c}
p_{1} \\
p_{2} \\
\vdots \\
q_{j} \\
\vdots \\
p_{N}
\end{array}\right\}=} \\
& {\left[\begin{array}{cccccc}
G_{11} & G_{12} & \ldots & -H_{1 j} & \ldots & G_{1 N} \\
G_{21} & G_{22} & \ldots & -H_{2 j} & \ldots & G_{2 N} \\
\vdots & \vdots & & \vdots & & \vdots \\
G_{N 1} & G_{N 2} & \ldots & -H_{N j} & \ldots & G_{N N}
\end{array}\right] \cdot\left\{\begin{array}{c}
\bar{q}_{1} \\
\bar{q}_{2} \\
\vdots \\
\bar{p}_{j} \\
\vdots \\
\bar{q}_{N}
\end{array}\right\}-\left\{\begin{array}{c}
D_{1} \\
D_{2} \\
\vdots \\
D_{j} \\
\vdots \\
D_{N}
\end{array}\right\}}
\end{aligned}
$$

Como no lado direito da igualdade só há valores conhecidos, é possível realizar o produto da matriz pelo vetor e subtrair o vetor resultante pelo vetor dos termos de domínio, obtendo assim um vetor chamado de $\{F\}$.

$$
[A] \cdot\{X\}=\{F\} \quad \text { (sistema matricial final de equações) }
$$

Assim, $\{X\}$ é o vetor das incógnitas e $[A]$ uma matriz de coeficientes conhecidos, relacionada às variáveis incógnitas do problema.

No caso de distribuições lineares, os nós são posicionados com afastamento de $0.25 \cdot L$ das extremidades do elemento para o centro, sendo $L$ o comprimento do elemento. Portanto, existem dois nós por elemento, gerando um sistema de equações com $2 \cdot N E$ variáveis incógnitas, a ser resolvido. $\mathrm{O}$ afastamento é necessário, quando se usa nós descontínuos, para evitar a geração de sistemas de equações incompatíveis.

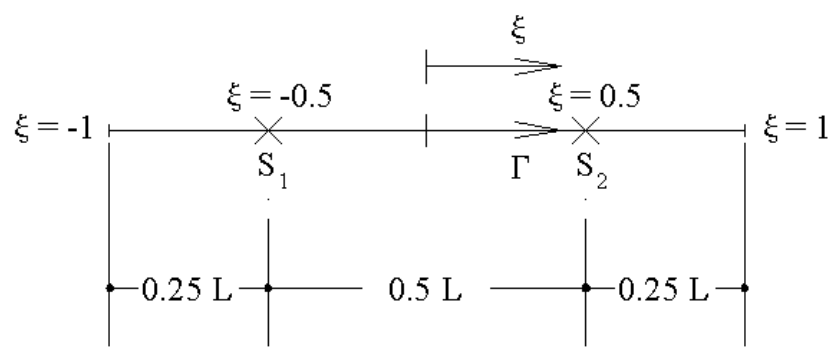

Figura 2.6 - Pontos de colocação no caso de elemento com distribuições lineares

A equação (2.22) passa a ser expressa em função dos nós: $N N D=2 \cdot N E$.

$$
c_{(S)} \cdot p_{i}+\int_{\Gamma} \frac{\partial p^{*}}{\partial \eta} \cdot \phi_{k} \cdot p d_{\Gamma}=\int_{\Gamma} p^{*} \cdot \phi_{k} \cdot q d_{\Gamma}-D_{i}
$$




$$
\begin{gathered}
c_{(S)} \cdot p_{i}+\sum_{j=1}^{N N D}\left(\int_{\Gamma} \frac{\partial p^{*}}{\partial \eta} d_{\Gamma}\right) \cdot \phi_{k} \cdot p_{j}=\sum_{j=1}^{N N D}\left(\int_{\Gamma} p^{*} d_{\Gamma}\right) \cdot \phi_{k} \cdot q_{j}-D_{i} \\
c_{(S)} \cdot p_{i}+\sum_{j=1}^{N N D} \hat{H}_{i j} \cdot \phi_{k} \cdot p_{j}=\sum_{j=1}^{N N D} G_{i j} \cdot \phi_{k} \cdot q_{j}-D_{i} \\
\sum_{j=1}^{N N D} H_{i j} \cdot \phi_{k} \cdot p_{j}=\sum_{j=1}^{N N D} G_{i j} \cdot \phi_{k} \cdot q_{j}-D_{i}
\end{gathered}
$$

Onde:

$$
\begin{aligned}
& i=1, N N D \text { e } k=1,2 \\
& H_{i j}=\begin{array}{ll}
\hat{H}_{i j} & \text { para } i \neq j \\
\hat{H}_{i j}+c_{(S) S \phi} & \text { para } i=j
\end{array} \\
& c_{(S) S \phi}=0 \text { para pontos } S \text { externos ao domínio. } \\
& c_{(S) S \phi}=1 \text { para pontos } S \text { internos ao domínio. }
\end{aligned}
$$

Quando o ponto de colocação estiver no contorno:

$$
\begin{aligned}
& c_{(S) 11}=\frac{1}{2} \cdot \phi_{1}=\frac{1}{2} \cdot \frac{1}{2} \cdot(1-\xi)=\frac{1}{4} \cdot[1-(-0.5)]=0.375 \\
& c_{(S) 12}=\frac{1}{2} \cdot \phi_{2}=\frac{1}{2} \cdot \frac{1}{2} \cdot(1+\xi)=\frac{1}{4} \cdot[1+(-0.5)]=0.125 \\
& c_{(S) 21}=\frac{1}{2} \cdot \phi_{1}=\frac{1}{2} \cdot \frac{1}{2} \cdot(1-\xi)=\frac{1}{4} \cdot(1-0.5)=0.125 \\
& c_{(S) 22}=\frac{1}{2} \cdot \phi_{2}=\frac{1}{2} \cdot \frac{1}{2} \cdot(1+\xi)=\frac{1}{4} \cdot(1+0.5)=0.375
\end{aligned}
$$

Neste caso, o valor $\frac{1}{2}$ que multiplica as funções de forma $\phi$ se deve ao fato de tanto para $S_{1}$ como para $S_{2}$, o ângulo formado no contorno $(\alpha)$ vale $\pi, \log 0 \frac{\alpha}{2 \pi}=\frac{\pi}{2 \pi}=\frac{1}{2}$.

\subsection{Técnicas de integração}

\subsubsection{Integração numérica}

O MEC é baseado numa equação integral de contorno, no caso (2.20). Qualquer equação integral possui termos integrais distintos em duas partes: núcleo da integra ${ }^{4}$, que é a parte integrável diretamente, e densidade da integral, que é a parte incógnita. Acompanhando 
o desenvolvimento da equação (2.20) para (2.23), percebe-se que os termos $\hat{H}_{i j}$ e $G_{i j}$, que em (2.24) são apresentados na forma matricial, são os núcleos das integrais. Para calcular os valores de $\hat{H}_{i j}$ e $G_{i j}$ é utilizada a técnica de integração numérica de Gauss. São utilizados 12 pontos de Gauss. O parâmetro adimensional $\xi$ adquiri importância dual, na definiçãa das funções de forma (item 2.3.1) e como limites de integração numérica. A técnica consiste em integrar a função desejada num intervalo definido por $\xi$, através de pontos discretos $\xi_{i g}$ e seus respectivos pesos $w_{i g}$. Inicialmente, o intervalo é tomado de -1 a 1 :

$$
\int_{-1}^{1} f_{(\xi)} d_{\xi} \cong \sum_{i g=1}^{N P G} f_{\left(\xi_{i g}\right)} \cdot w_{i g}
$$

Para intervalos genéricos de integração numérica, por exemplo de $L_{1}$ a $L_{2}$, utilizase a seguinte fórmula:

$$
\int_{L_{1}}^{L_{2}} f_{(\Gamma)} d_{\Gamma}=\left(\frac{L_{2}-L_{1}}{2}\right) \cdot \int_{-1}^{1} f_{\left(\left(\frac{L_{2}+L_{1}}{2}\right)+\frac{\xi}{2} \cdot\left(L_{2}-L_{1}\right)\right)} d_{\xi}
$$

Quando $\Gamma$ e $\xi$ coincidem com o ponto médio do elemento onde ocorre a integração, $L_{1}=-\frac{L}{2}$ e $L_{2}=\frac{L}{2}$, pode-se utilizar a equação (2.31).

$$
\int_{-\frac{L}{2}}^{\frac{L}{2}} f_{(\Gamma)} d_{\Gamma}=\left[\frac{\frac{L}{2}-\left(-\frac{L}{2}\right)}{2}\right] \cdot \int_{-1}^{1} f_{\left(\left(\frac{\frac{L}{2}-\frac{L}{2}}{2}\right)+\frac{\xi}{2} \cdot\left[\frac{L}{2}-\left(-\frac{L}{2}\right)\right]\right)} d_{\xi}=\frac{L}{2} \cdot \int_{-1}^{1} f_{\left(\xi \frac{L}{2}\right)} d_{\xi}
$$

O caso da integração numérica de Gauss chamada de logarítmica é útil quando as soluções fundamentais a serem integradas possuem singularidades do tipo logarítmica. Vale destacar que a solução fundamental apresentada em (2.21) é expressa em termos da função de Neumann $\left(Y_{n}\right)$; quando representada na forma de série, $Y_{n}$ contém um termo logarítmico. A integração numérica logarítmica, num intervalo de 0 a 1 é expressa pela fórmula abaixo.

$$
\int_{0}^{1} \ln \left(\frac{1}{\xi}\right) \cdot f_{(\xi)} d_{\xi} \cong \sum_{i g l=1}^{N P G l} f_{\left(\xi_{i g l}\right)} \cdot w_{i g l}
$$

São utilizados 10 pontos de Gauss na integração numérica logarítmica.

As tabelas contendo os pontos de integração $\xi_{i g}$ e $\xi_{i g l}$ com seus pesos $w_{i g}$ e $w_{i g l}$, podem ser encontradas em ABRAMOWITZ \& STEGUN (1972).

\footnotetext{
${ }^{4}$ Também chamado de kernel.
} 


\subsubsection{Ponto de colocação quase singular}

Quando os pontos de colocação são posicionados fora do domínio, afastados uma distância $d$ do elemento de contorno segundo sua reta normal $\eta$, a integração no elemento de onde o ponto de colocação foi tomado, é dita quase singular. Como o ponto de colocação não pertence ao elemento, não existe singularidade. É importante destacar que as posições dos pontos de colocação não devem coincidir, pois o sistema de equações pode se tornar incompatível.

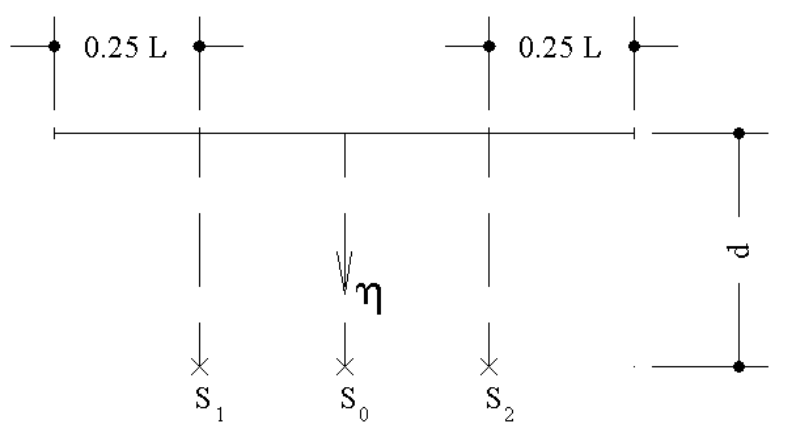

Figura 2.7 - Pontos de colocação posicionados fora do domínio

Da figura 2.6 tira-se a relação: $\Gamma=\frac{L}{2} \cdot \xi$. Os núcleos das equações integrais são calculados por integração numérica de Gauss:

$$
\begin{gathered}
G_{i j}=\int_{\Gamma} p^{*} \cdot \phi_{k} d_{\Gamma}=\int_{\Gamma} \frac{1}{4} \cdot\left[i \cdot J_{0}(K R)-Y_{0}(K R)\right] \cdot \phi_{k} d_{\Gamma} \\
=\frac{1}{4} \cdot \int_{-1}^{1}\left[i \cdot J_{0}\left(K R_{(\xi)}\right)-Y_{0}\left(K R_{(\xi)}\right)\right] \cdot \phi_{k} \cdot \frac{L}{2} d_{\xi}= \\
=\frac{L}{8} \cdot \sum_{i g=1}^{N P G}\left[i \cdot J_{0}\left(K R_{\left(\xi_{i g}\right)}\right)-Y_{0}\left(K R_{\left(\xi_{i g}\right)}\right)\right] \cdot \phi_{k} \cdot w_{i g} \\
\hat{H}_{i j}=\int_{\Gamma} q^{*} \cdot \phi_{k} d_{\Gamma}=\int_{\Gamma}-\frac{K}{4} \cdot\left[i \cdot J_{1}(K R)-Y_{1}(K R)\right] \cdot \frac{\partial R}{\partial \eta} \cdot \phi_{k} d_{\Gamma} \\
=-\frac{K}{4} \cdot \int_{-1}^{1}\left[i \cdot J_{1}\left(K R_{(\xi)}\right)-Y_{1}\left(K R_{(\xi)}\right)\right] \cdot R,_{\eta} \cdot \phi_{k} \cdot \frac{L}{2} d_{\xi}= \\
=-\frac{K \cdot L}{8} \cdot \sum_{i g=1}^{N P G}\left[i \cdot J_{1}\left(K R_{\left(\xi_{i g}\right)}\right)-Y_{1}\left(K R_{\left(\xi_{i g}\right)}\right)\right] \cdot R,,_{\eta} \cdot \phi_{k} \cdot w_{i g}
\end{gathered}
$$

Onde:

$\phi_{k}=1$ para distribuições constantes.

$k=1,2$ para distribuições lineares: $\phi_{1}=\frac{1}{2} \cdot(1-\xi)$ e $\phi_{2}=\frac{1}{2} \cdot(1+\xi)$. 


$$
R_{,_{\eta}}=\frac{\partial R}{\partial \eta}=\frac{\partial R}{\partial X} \cdot \frac{\partial X}{\partial \eta}+\frac{\partial R}{\partial Y} \cdot \frac{\partial Y}{\partial \eta}=R,_{X} \cdot \eta_{X}+R,_{Y} \cdot \eta_{Y}
$$

$R_{\left(\xi_{(i g)}\right)}$ é a distância dos pontos de Gauss ao ponto de colocação $S$.

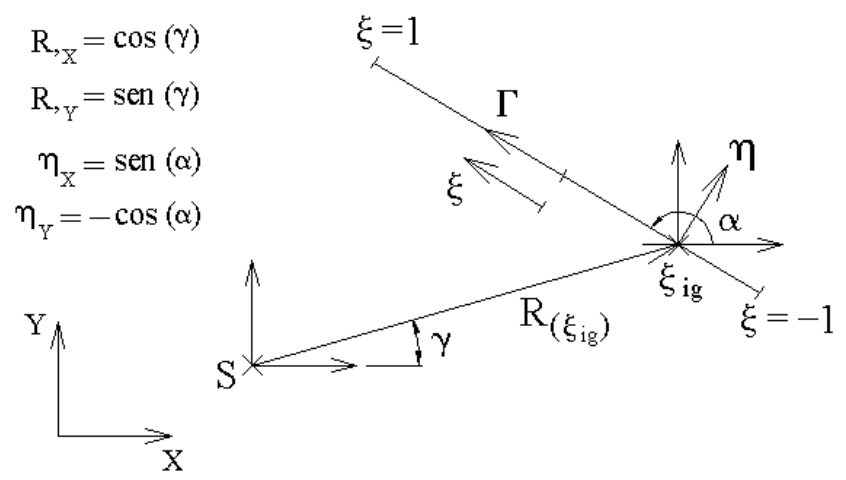

Figura 2.8 - Representação gráfica das variáveis geométricas envolvidas na integração

\subsubsection{Sub-elementação}

Quando a técnica de integração com ponto de colocação quase singular é utilizada e a distância de afastamento $d$ for pequena, é necessário subdividir os elementos que estiverem próximos ao ponto de colocação para se obter bons resultados na integração numérica. $\mathrm{O}$ critério adotado para a aplicação da sub-elementação é baseado nas distâncias dos nós inicial $(n i)$ e final $(n f)$ do elemento em que ocorre a integração ao ponto de colocação $S$. A distância de $S$ a $n i$ é chamada $a 1$ e de $S$ a $n f$ é chamada $a 2$. Quando $a 1$ ou $a 2$ forem menores que o comprimento do elemento $L$, a sub-elementação é utilizada.

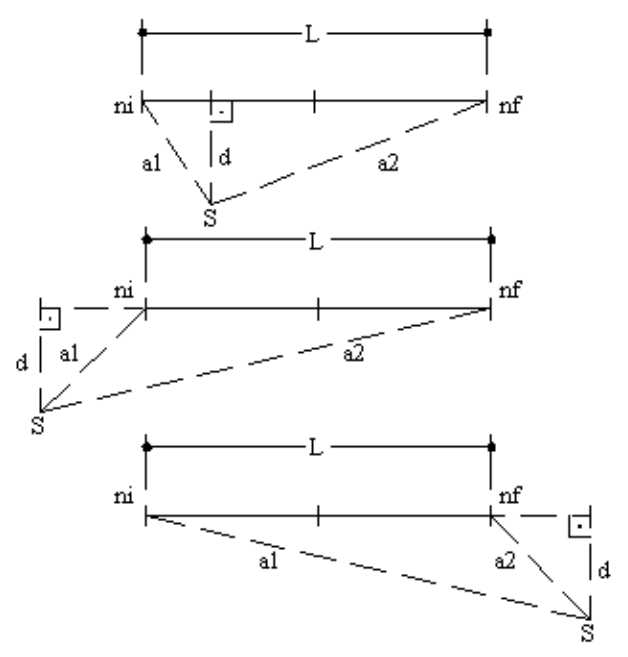

Figura 2.9 - Posições do ponto de colocação em relação ao elemento 
A sub-elementação utilizada é do tipo constante, ou seja, os sub-elementos possuem tamanhos pré definidos, iguais a metade da distância $d$. A divisão em sub-elementos começa a partir do nó inicial do elemento; como conseqüência, o último sub-elemento pode ter tamanho diferente dos demais, sendo igual ao trecho que falta para completar o elemento. A soma das integrações nos sub-elementos, em relação ao ponto de colocação, fornece o núcleo integral $\left(\hat{H}_{i j}\right.$ ou $\left.G_{i j}\right)$ procurado.

Para realizar as integrações nos sub-elementos é necessário recorrer à fórmula de mudança nos limites de integração (2.31).

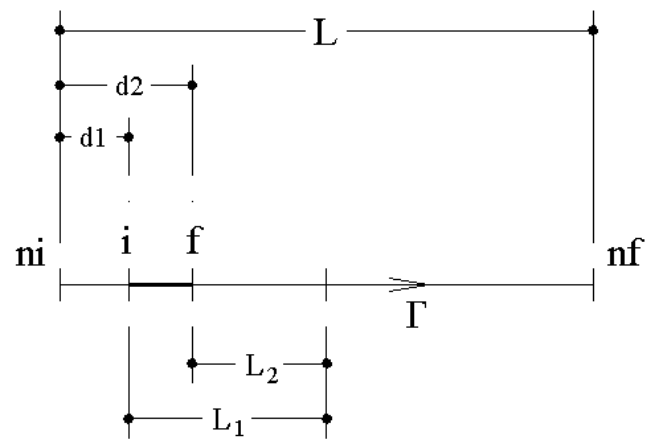

Figura 2.10 - Limites de integração de um sub-elemento

As variáveis $d 1$ e $d 2$ são importantes para se definir o sinal de $L_{1}$ e $L_{2}$ em relação a $\Gamma$. Os limites de integração adimensionais são ilustrados na figura abaixo.

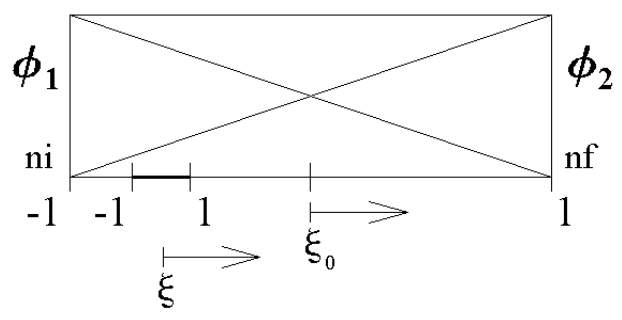

Figura 2.11 - Limites adimensionais de integração do elemento e do sub-elemento

As mudanças nos limites de integração levam a uma transformação do tipo $f_{\left(\xi_{0}\right)}=f_{\left(\frac{2}{L} \cdot \Gamma\right)}=f_{\left(\frac{2}{L} \cdot\left(\left(\frac{L_{2}+L_{1}}{2}\right)+\frac{\xi}{2} \cdot\left(L_{2}-L_{1}\right)\right)\right)}=f_{\left(\left(\frac{L_{2}+L_{1}}{L}\right)+\frac{\xi}{L} \cdot\left(L_{2}-L_{1}\right)\right)}$. Portanto, as funções de forma para o caso de distribuições lineares apresentado na figura 2.11 são modificadas em função dos novos limites de $\xi$. 


$$
\begin{aligned}
& \phi_{1}=\frac{1}{2} \cdot\left(1-\xi_{0}\right)=\frac{1}{2} \cdot\left[1-\left(\left(\frac{L_{2}+L_{1}}{L}\right)+\frac{\xi}{L} \cdot\left(L_{2}-L_{1}\right)\right)\right] \\
& \phi_{2}=\frac{1}{2} \cdot\left(1+\xi_{0}\right)=\frac{1}{2} \cdot\left[1+\left(\left(\frac{L_{2}+L_{1}}{L}\right)+\frac{\xi}{L} \cdot\left(L_{2}-L_{1}\right)\right)\right]
\end{aligned}
$$

Com o procedimento de sub-elementação apresentado, garante-se que a integração nos elementos é precisa para pontos de colocação razoavelmente próximos dos elementos. Quanto menor a distância de afastamento do ponto de colocação em relação ao elemento, com o uso de sub-elementação, melhor o resultado da integração. Porém é conveniente alertar que distâncias muito pequenas demandam maior tempo de processamento computacional, e dependendo das grandezas envolvidas no problema, podem-se perder valores por truncamento e aproximações feitas pelo processador do computador utilizado.

\subsubsection{Ponto de colocação no contorno}

Neste caso, os núcleos integrais no elemento a que o ponto de colocação pertence são singulares. Pelo fato dos elementos de contorno serem lineares, o núcleo integral $\hat{H}_{i j}$ é igual a zero, pois em qualquer ponto do elemento, o vetor $R_{(S, P)}$ é perpendicular à reta normal $\eta: \frac{\partial R}{\partial \eta}=0 \Rightarrow \hat{H}_{i j}=0$. Lembrando-se que $\frac{\partial R}{\partial \eta}$ é coeficiente multiplicativo na equação de $\hat{H}_{i j}$, (2.34). Portanto, a singularidade fica restrita ao núcleo $G_{i j}$, e ocorre quando $R=0 . G_{i j}$ é constituído de uma parcela não definida em zero: $Y_{0}(0) \rightarrow-\infty$.

Para resolver o problema da singularidade, utiliza-se um esquema de integração numérica logarítmica. Multiplicando e dividindo o núcleo integral por $\ln \left(\frac{1}{\xi}\right)$ é possível realizar a integração numérica diretamente, de zero à uma extremidade do elemento, portanto o elemento é dividido em dois intervalos. Optou-se pela integração logarítmica, ao invés da integração numérica convencional, pela facilidade em separar o elemento em dois trechos de integração.

(a) Distribuições constantes 


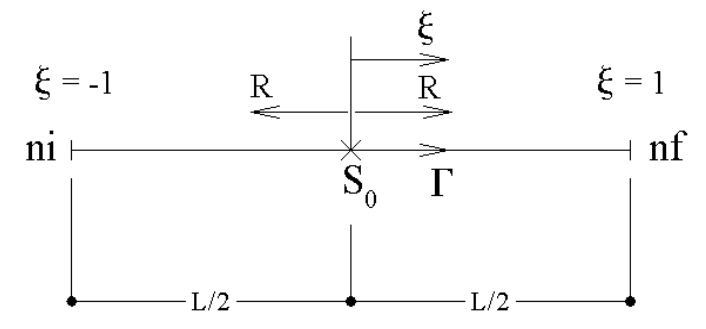

Figura 2.12 - Coordenadas locais envolvidas na integração singular do elemento com distribuições constantes

$$
\begin{aligned}
G_{i j} & =\frac{1}{4} \cdot \int_{-\frac{L}{2}}^{\frac{L}{2}}\left[i \cdot J_{0}(K R)-Y_{0}(K R)\right] d_{\Gamma} \\
& =\frac{1}{4} \cdot \frac{L}{2} \cdot \int_{-1}^{1} i \cdot J_{0}\left(K \cdot \frac{L}{2} \cdot \xi\right) d_{\xi}-\frac{1}{4} \cdot 2 \cdot \int_{0}^{\frac{L}{2}} Y_{0}(K R) d_{R}=I N T 1+I N T 2
\end{aligned}
$$

Utiliza-se a técnica de integração numérica de Gauss convencional para INT1:

$$
I N T 1=\frac{L}{8} \cdot \sum_{i g=1}^{N P G} i \cdot J_{0}\left(\frac{K L}{2} \cdot \xi_{i g}\right) \cdot w_{i g}
$$

Para o cálculo de INT2 é aplicada a integração numérica logarítmica:

$$
\begin{aligned}
I N T 2 & =-\frac{1}{2} \cdot \int_{0}^{1} Y_{0}\left(\frac{K L}{2} \cdot \xi\right) \cdot \frac{L}{2} \cdot \frac{\ln \left(\frac{1}{\xi}\right)}{\ln \left(\frac{1}{\xi}\right)} d_{\xi} \\
& =-\frac{L}{4} \cdot \sum_{i g l=1}^{N P G l}\left[\frac{Y_{0}\left(\frac{K L}{2} \cdot \xi_{i g l}\right)}{-\ln \left(\xi_{i g l}\right)} \cdot w_{i g l}\right]=\frac{L}{4} \cdot \sum_{i g l=1}^{N P G l}\left[\frac{Y_{0}\left(\frac{K L}{2} \cdot \xi_{i g l}\right)}{\ln \left(\xi_{i g l}\right)} \cdot w_{i g l}\right]
\end{aligned}
$$

(b) Distribuições lineares

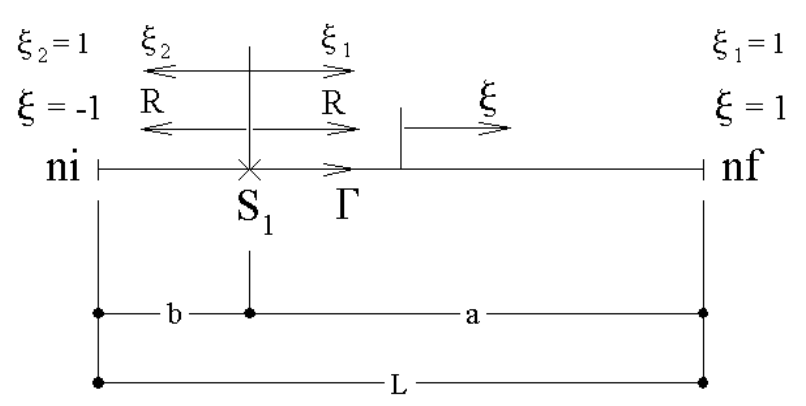

Figura 2.13 - Coordenadas locais envolvidas na integração singular do elemento com distribuições lineares 


$$
\begin{aligned}
G_{i j} & =\frac{1}{4} \cdot \int_{-b}^{a}\left[i \cdot J_{0}(K R)-Y_{0}(K R)\right] \cdot \phi_{k} d_{\Gamma} \\
& =\frac{1}{4} \cdot \int_{-b}^{a} i \cdot J_{0}(K R) \cdot \phi_{k} d_{\Gamma}-\frac{1}{4} \cdot \int_{0}^{a} Y_{0}(K R) \cdot \phi_{k} d_{\Gamma}-\frac{1}{4} \cdot \int_{-b}^{0} Y_{0}(K R) \cdot \phi_{k} d_{\Gamma} \\
& =I N T 1+I N T 2+I N T 3
\end{aligned}
$$

Para o cálculo de INT1, integração numérica convencional, trabalha-se no sistema de coordenadas adimensional $\xi$. Tem-se a transformação: $f_{(R)}=f_{\left(\left|\frac{a-b}{2}+\frac{L}{2} \cdot \xi\right|\right)}$, obtida pela mudança nos limites de integração, fórmula (2.31).

$$
\begin{aligned}
& I N T 1=\frac{1}{4} \cdot \int_{-1}^{1} i \cdot J_{0}\left(K \cdot\left[\left|\frac{a-b}{2}+\frac{L}{2} \cdot \xi\right|\right]\right) \cdot \phi_{k} \cdot \frac{L}{2} d_{\xi} \\
& =\frac{L}{8} \cdot \sum_{i g=1}^{N P G} i \cdot J_{0}\left(K \cdot\left[\left|\frac{a-b}{2}+\frac{L}{2} \cdot \xi_{i g}\right|\right]\right) \cdot \phi_{k} \cdot w_{i g}
\end{aligned}
$$

As integrais INT2 e INT3 são calculadas nos trechos de comprimentos $a$ e $b$, respectivamente.

Para o cálculo de INT2, $\Gamma \in] 0, a]$, utiliza-se o sistema de coordenadas adimensionais $\xi_{1}$, tal que $R=a \cdot \xi_{1} \Rightarrow d_{R}=a \cdot d_{\xi_{1}}$. As funções de forma podem ser escritas da seguinte maneira:

$$
\begin{aligned}
& \phi_{1}=\frac{a-R}{a+b}=\frac{a \cdot\left(1-\xi_{1}\right)}{L} \\
& \phi_{2}=\frac{b+R}{a+b}=\frac{b+a \cdot \xi_{1}}{L}
\end{aligned}
$$

Calcula-se INT2 por integração numérica logarítmica:

$$
\begin{aligned}
I N T 2 & =-\frac{1}{4} \cdot \int_{0}^{1} Y_{0}\left(K \cdot a \cdot \xi_{1}\right) \cdot \phi_{k} \cdot a \cdot \frac{\ln \left(\frac{1}{\xi_{1}}\right)}{\ln \left(\frac{1}{\xi_{1}}\right)} d_{\xi_{1}} \\
& =-\frac{a}{4} \cdot \sum_{i g l=1}^{N P G l}\left[\frac{Y_{0}\left(K \cdot a \cdot \xi_{\text {ligl }}\right)}{-\ln \left(\xi_{1 i g l}\right)} \cdot \phi_{k} \cdot w_{i g l}\right] \\
& =\frac{a}{4} \cdot \sum_{i g l=1}^{N P G l}\left[\frac{Y_{0}\left(K \cdot a \cdot \xi_{\text {ligl }}\right)}{\ln \left(\xi_{\text {ligl }}\right)} \cdot \phi_{k} \cdot w_{i g l}\right]
\end{aligned}
$$


Para o cálculo de INT3, $\Gamma \in[-b, 0[$, utiliza-se o sistema de coordenadas adimensionais $\xi_{2}$, tal que $R=b \cdot \xi_{2} \Rightarrow d_{R}=b \cdot d_{\xi_{2}}$. As funções de forma são escritas como:

$$
\begin{aligned}
& \phi_{1}=\frac{a+R}{a+b}=\frac{a+b \cdot \xi_{2}}{L} \\
& \phi_{2}=\frac{b-R}{a+b}=\frac{b \cdot\left(1-\xi_{2}\right)}{L}
\end{aligned}
$$

Calcula-se INT3 :

$$
\begin{aligned}
\text { INT3 } & =\frac{1}{4} \cdot \int_{0}^{-b} Y_{0}(K R) \cdot \phi_{k} d_{\Gamma}=\frac{1}{4} \cdot \int_{0}^{b} Y_{0}(K R) \cdot \phi_{k} d_{R} \\
& =\frac{1}{4} \cdot \int_{0}^{1} Y_{0}\left(K \cdot b \cdot \xi_{2}\right) \cdot \phi_{k} \cdot b \cdot \frac{\ln \left(\frac{1}{\xi_{2}}\right)}{\ln \left(\frac{1}{\xi_{2}}\right)} d_{\xi_{2}} \\
& =\frac{b}{4} \cdot \sum_{i g l=1}^{N P G l}\left[\frac{Y_{0}\left(K \cdot b \cdot \xi_{2 i g l}\right)}{-\ln \left(\xi_{2 i g l}\right)} \cdot \phi_{k} \cdot w_{i g l}\right] \\
& =-\frac{b}{4} \cdot \sum_{i g l=1}^{N P G l}\left[\frac{Y_{0}\left(K \cdot b \cdot \xi_{2 i g l}\right)}{\ln \left(\xi_{2 i g l}\right)} \cdot \phi_{k} \cdot w_{i g l}\right]
\end{aligned}
$$

Nota-se que no trecho de comprimento $a, d_{R}=d_{\Gamma}$. Já no trecho de comprimento $b, d_{R}=-d_{\Gamma}$. Portanto na mudança da variável de integração de $\Gamma$ para $R$, é lícito escrever $\int_{0}^{-b} d_{\Gamma}=\int_{0}^{b} d_{R}$. Propriedade semelhante foi utilizada no caso das distribuições constantes (2.36), de forma implícita.

Quando o ponto de colocação não está no elemento de contorno integrado, não há singularidade. A integração é feita da mesma maneira descrita no conjunto de equações (2.34).

\subsubsection{Aplicação do CHIEF}

Quando a técnica de integração com ponto de colocação no contorno é utilizada para analisar domínios infinitos, a equação integral de contorno (2.20) apresenta um problema de não unicidade de resposta. Na realidade, cada número de ondas $K$ apresenta resposta única em pressão e fluxo de pressão. Portanto, a não unicidade é um problema matemático, gerado pelo tipo de formulação integral de contorno utilizada. Segundo KANE (1994), o erro 
numérico ocorre quando as freqüências de vibração do domínio infinito se aproximam das freqüências naturais do domínio finito, com o contorno comum aos dois domínios. Vale lembrar que a freqüência é diretamente proporcional ao número de ondas.

Os resultados das integrações singulares em problemas de domínio infinito, podem gerar não unicidade ou mal condicionamento de resposta em potencial e fluxo de pressão. Para resolver este problema, utiliza-se a técnica CHIEF (Combined Helmholtz Integral Equation Formulation). A formulação do CHIEF é baseada no método dos mínimos quadrados, usado para ajustar curvas a partir de pontos dados, de maneira a minimizar o erro. O método dos mínimos quadrados pode ser encontrado em PIPES \& HARVILL (1970). A técnica consiste em introduzir equações adicionais no sistema algébrico, obtidas pela consideração de pontos de colocação complementares fora do domínio. Portanto, o sistema é escrito com mais equações do que incógnitas. Matricialmente, são adicionadas novas linhas de coeficientes nas matrizes $[H]$ e $[G]$. Neste trabalho, o CHIEF não é aplicado em casos onde há termo de domínio, $\{D\}$.

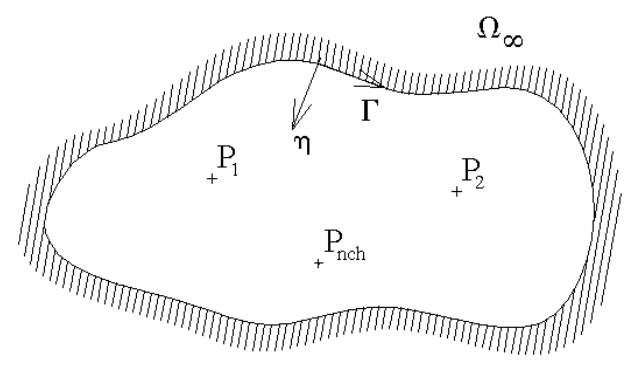

Figura 2.14 - Pontos de CHIEF para um domínio infinito

Considerando-se o sistema na forma matricial, com $N$ equações e $N$ incógnitas:

$$
[H]_{N \times N} \cdot\{p\}_{N}=[G]_{N \times N} \cdot\{q\}_{N}
$$

Acrescentando $n c h$ pontos de colocação adicionais fora do domínio, chamados de pontos de CHIEF, o sistema passa a ter $M$ equações, $M=N+n c h$, e $N$ incógnitas. As matrizes $[H]$ e $[G]$ ampliadas, passam a ser chamadas de $[H c]$ e $[G c]$.

$$
[H c]_{M \times N} \cdot\{p\}_{N}=[G c]_{M \times N} \cdot\{q\}_{N}
$$

Para retornar o sistema ao número de equações inicial, multiplica-se o sistema (2.46) por $[H c]^{T}$.

$$
[H c]_{N \times M}^{T} \cdot[H c]_{M \times N} \cdot\{p\}_{N}=[H c]_{N \times M}^{T} \cdot[G c]_{M \times N} \cdot\{q\}_{N}
$$

O sistema modificado é rescrito por duas novas matrizes, chamadas de $[H n]$ e $[G n]$. 


$$
\begin{gathered}
{[H n]_{N \times N} \cdot\{p\}_{N}=[G n]_{N \times N} \cdot\{q\}_{N}} \\
{[A]_{N \times N} \cdot\{X\}_{N}=\{F\}_{N} \quad \text { (sistema final de equações modificado) }}
\end{gathered}
$$

\subsection{Potenciais de pressão em pontos internos ao domínio}

Depois de calculadas as variáveis incógnitas do contorno pelo MEC, podem-se obter valores de potenciais de pressão em pontos internos ao domínio. A partir da equação (2.20), chega-se à seguinte relação:

$$
p_{\text {int }}=\int_{\Gamma} p^{*} \cdot \frac{\partial p}{\partial \eta} d_{\Gamma}-\int_{\Gamma} \frac{\partial p^{*}}{\partial \eta} \cdot p d_{\Gamma}-\int_{\Omega} D \cdot p^{*} d_{\Omega}
$$

Na forma matricial:

$$
p_{\text {int }}=[G]_{1 \times N} \cdot\{q\}_{N}-\lfloor\hat{H}\rfloor_{1 \times N} \cdot\{p\}_{N}-\{D\}_{1}
$$

Onde $N$ indica o número de variáveis de pressão ou fluxo de pressão no contorno, depende do tipo de distribuição utilizada: constante $(N=N E)$ ou linear $(N=N N D)$.

Os núcleos integrais $\hat{H}_{1 j}$ e $G_{1 j}$ são calculados tomando-se como ponto de colocação apenas o ponto interno analisado, por isso é gerada apenas uma linha nas matrizes $\lfloor\hat{H}\rfloor$ e $[G]$. O esquema de integração é semelhante ao utilizado no conjunto de equações (2.34), portanto, não há singularidades no cálculo dos núcleos integrais. Da mesma maneira, a contribuição do termo de domínio $\{D\}$ é constituída apenas de um valor, gerando uma grandeza escalar $D_{1}$.

\subsection{Tipos de termos de domínio considerados}

Neste trabalho serão considerados dois tipos de termos de domínio: núcleo de pressão concentrado em um ponto $\left(D_{P}\right)$ e núcleo de pressão constante distribuído em linha $\left(D_{L}\right)$. 


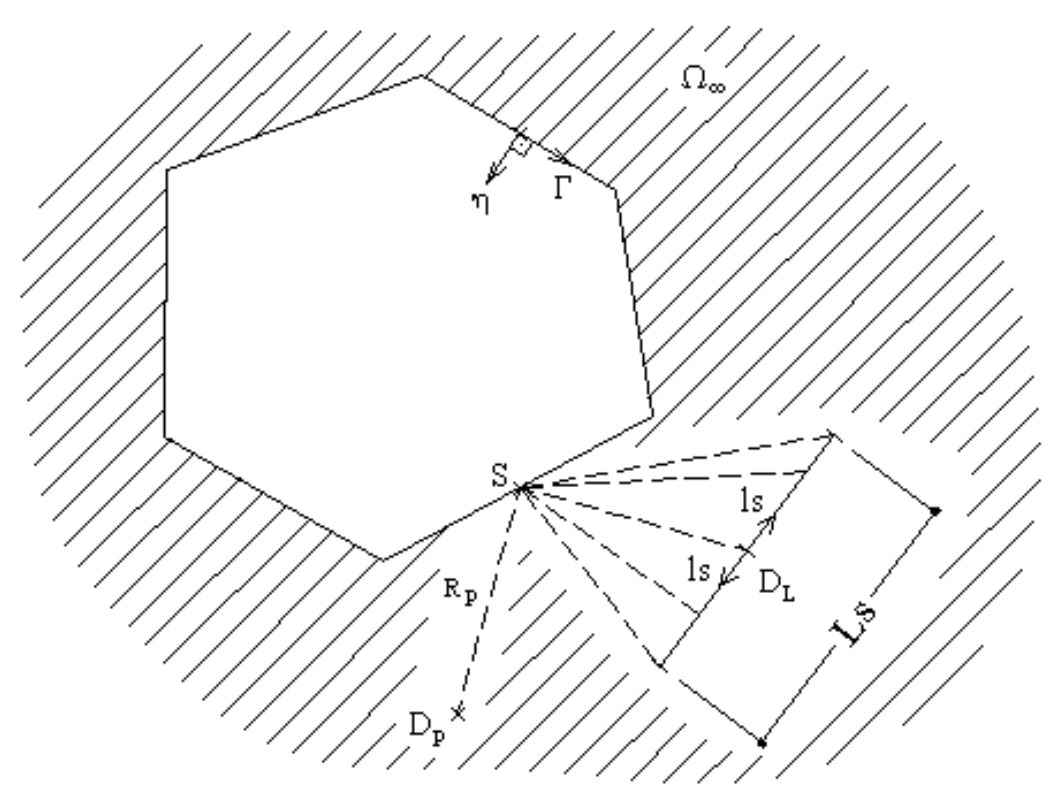

Figura 2.15 - Núcleos de pressão em um domínio infinito

(a) Núcleo de pressão concentrado em um ponto:

$$
\{D\}=\int_{\Omega} D \cdot p^{*} d_{\Omega}=D_{P} \cdot p^{*}=\frac{D_{P}}{4} \cdot\left[i \cdot J_{0}\left(K R_{P}\right)-Y_{0}\left(K R_{P}\right)\right]
$$

(b) Núcleo de pressão constante distribuído em linha:

$$
\begin{aligned}
\{D\} & =\int_{\Omega} D \cdot p^{*} d_{\Omega}=\int_{l s} D_{L} \cdot \frac{1}{4} \cdot\left[i \cdot J_{0}\left(K R_{l s}\right)-Y_{0}\left(K R_{l s}\right)\right] d_{l s} \\
& =\frac{D_{L}}{4} \cdot \int_{-\frac{L s}{2}}^{\frac{L s}{2}}\left[i \cdot J_{0}\left(K R_{l s}\right)-Y_{0}\left(K R_{l s}\right)\right] d_{l s}
\end{aligned}
$$

Como a integração é feita em $\int_{l s} p^{*} d_{l s}$, depende apenas de $K$ e $R_{l s}$. Portanto, tanto faz o sentido $l s$ utilizado na integração numérica da linha $L s$.

Dimensionalmente, com base na equação (2.5), o termo de domínio $\{D\}$ tem unidade de pressão por distância ao quadrado. Por exemplo, se o sistema de medidas adotado for o internacional (SI), $\{\mathrm{D}\}$ terá como unidade $\left[\mathrm{Pa} / \mathrm{m}^{2}\right]$. 


\section{EXEMPLOS NUMÉRICOS DE PROBLEMAS DE RADIAÇÃO E DIFUSÃO ACÚSTICA BIDIMENSIONAL}

\subsection{Considerações iniciais}

Para os exemplos numéricos analisados serão utilizadas soluções e técnicas de integração diferentes, listadas na tabela 3.1. As respostas serão apresentadas na forma de módulo, lembrando que as variáveis envolvidas na formulação do problema estão na forma de números complexos. Os gráficos apresentados neste capítulo serão feitos para pontos discretos de número de ondas, portanto pontos de descontinuidade, devidos à não unicidade de resposta em domínios infinitos e freqüências naturais em domínios finitos, não serão rastreados no espectro de respostas.

Tabela 3.1 - Tipos de soluções e técnicas de integração

\begin{tabular}{||l|l||}
\hline Analítica & Solução analítica do problema \\
\hline Equação integral & Solução analítica da equação integral (2.20) \\
\hline ANSYS & Solução pelo programa ANSYS 5.5.1 \\
\hline TC1 $(d)$ & Integração com ponto de colocação fora do domínio \\
\hline TC2 & Integração com ponto de colocação no contorno \\
\hline TC3 & Integração com ponto de colocação no contorno, corrigida com CHIEF \\
\hline
\end{tabular}

Em TC1, (d) indica a distância de afastamento do ponto de colocação ao elemento.

Nos quatro primeiros exemplos estuda-se o caso de um cilindro de raio unitário imerso em um fluido infinito, com fluxo de pressão também unitário prescrito no contorno. Analisam-se os potenciais de pressão ao longo do contorno, em um intervalo de números de ondas de $1 \mathrm{a}$ 30; é utilizado apenas um ponto de CHIEF no centro do cilindro. O resultado dos potenciais apresentado nos gráficos é tomado como a média aritmética dos módulos de pressão nos 
elementos de contorno. A solução analítica para o problema de radiação de um cilindro pulsante imerso em um domínio infinito é encontrada em YOON et al. (1990):

$$
p=A_{0} \cdot\left[J_{0}(K R)-i \cdot Y_{0}(K R)\right]
$$

Onde:

$$
A_{0}=\frac{4 \cdot U_{a}}{K \cdot C_{0}^{2}} \cdot\left[J_{1}(K a)-i \cdot Y_{1}(K a)\right]
$$

Fluxo de pressão unitário: $U_{a}=\frac{\partial p}{\partial \eta}=1$.

Raio do cilindro: $a=1$.

Constantes adimensionais: $C_{0}=\frac{2 \cdot J_{1}(K a)}{\operatorname{sen}\left(\gamma_{0}\right)} ; \gamma_{0}=\operatorname{artcg}\left(-\frac{J_{1}(K a)}{Y_{1}(K a)}\right)$.

Da equação (3.1) obtém-se o valor $|p|$, que servirá como base de comparação para os quatro primeiros exemplos.

\subsection{Exemplo 1}

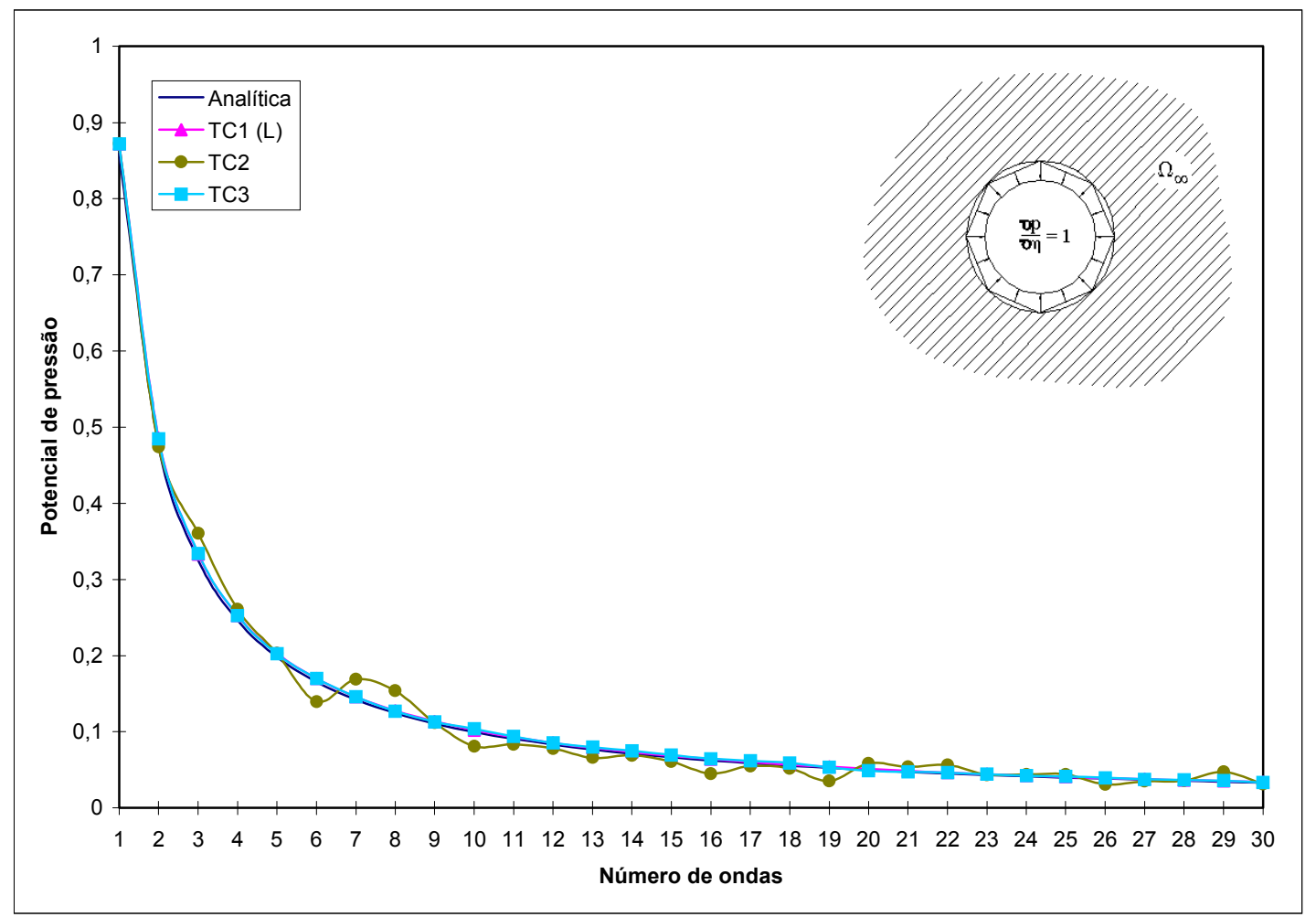

Figura 3.1 - Número de ondas $\times$ potencial de pressão (DC_8) 
O contorno é discretizado em 8 elementos lineares, com distribuições constantes de potencial e fluxo de pressão (DC_8).

Apesar de ter sido usada uma discretização pobre, foram obtidos bons resultados em termos de potencial de pressão no contorno. A técnica de integração TC2 apresentou desvios em vários pontos. Quando é aplicado o CHIEF, TC3, a resposta obtida praticamente se iguala à solução analítica. Resultado de qualidade semelhante se obtém quando é aplicada a técnica de integração TC1.

\subsection{Exemplo 2}

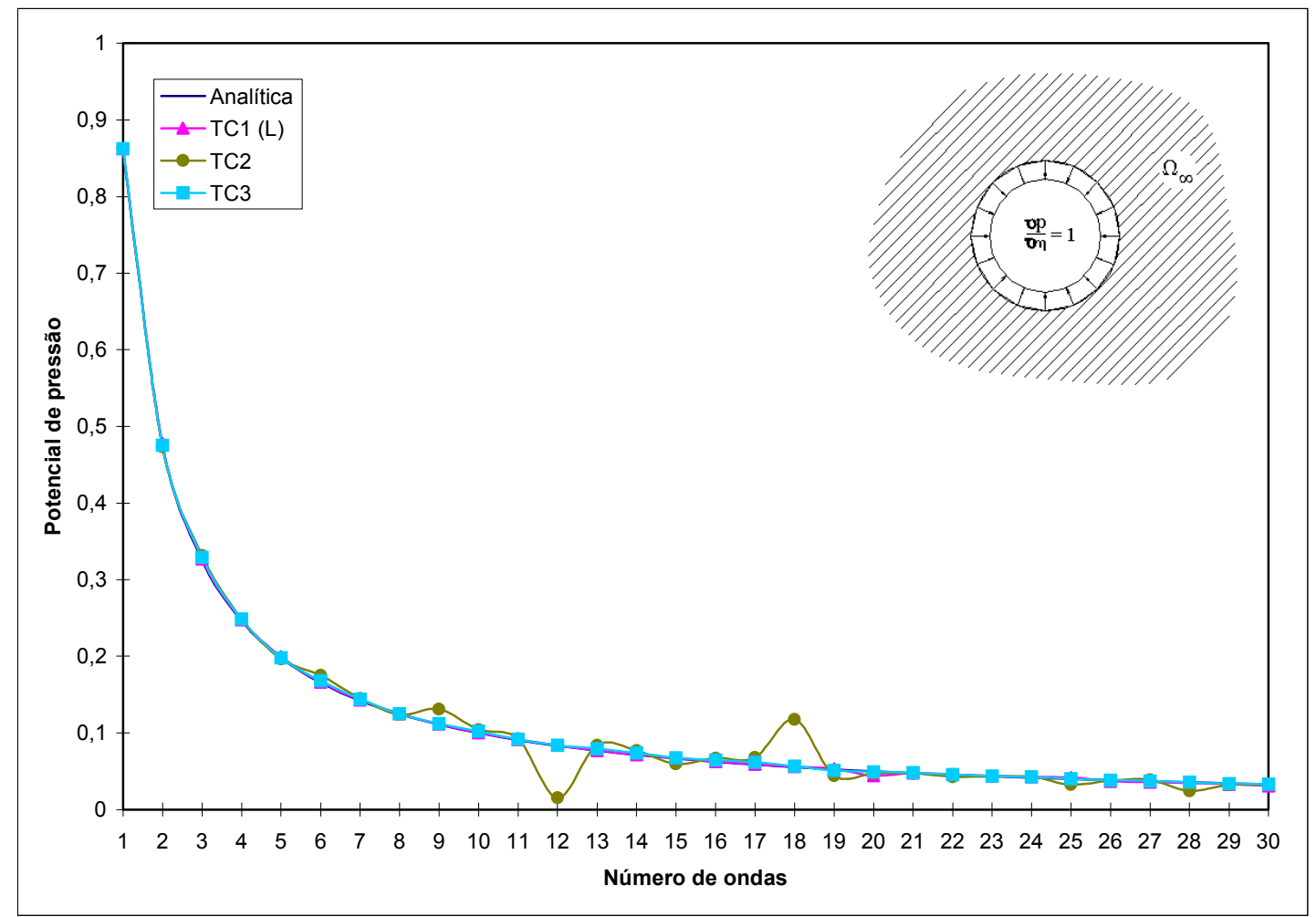

Figura 3.2 - Número de ondas $\times$ potencial de pressão (DC_16)

O contorno é discretizado em 16 elementos lineares, com distribuições constantes de potencial e fluxo de pressão (DC_16).

Uma discretização mais refinada do contorno, o dobro do exemplo 1, também forneceu resultados próximos da solução analítica para as técnicas TC1 e TC3. Para a análise utilizando TC2, não houve melhoria significativa de resultando, porque localmente os desvios foram maiores que no exemplo anterior, caso dos números de ondas 12 e 18 . 


\subsection{Exemplo 3}

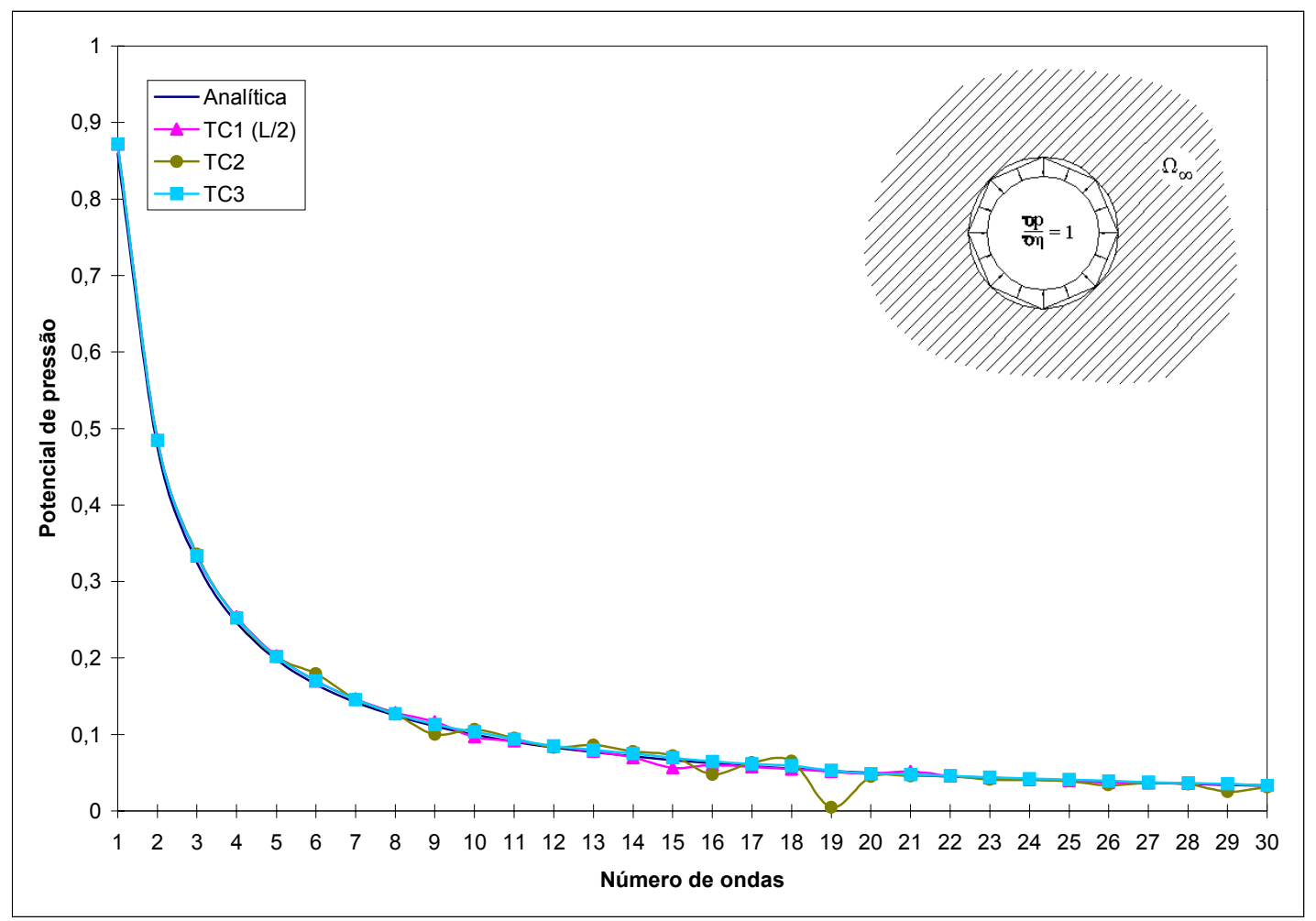

Figura 3.3 - Número de ondas $\times$ potencial de pressão (DL_8)

O contorno é discretizado em 8 elementos lineares, com distribuições lineares de potencial e fluxo de pressão (DL_8).

A técnica TC1 apresentou um pequeno desvio no número de ondas 15, para TC3 os valores foram praticamente iguais aos da solução analítica; para TC2 houve um desvio local de grande amplitude no número de ondas 19, mas globalmente os resultados foram melhores que os obtidos no exemplo 1, que possui a mesma discretização.

Comparando-se os resultados obtidos através da técnica de integração TC1 nos exemplos 1 e 3, percebe-se que o exemplo 1 apresenta resultados ligeiramente mais próximos da solução analítica. Este fato é devido à descontinuidade de potenciais nas extremidades dos elementos com distribuições lineares, discutida no item 2.3.1, que causa pequenos desvios na hora de calcular as médias dos módulos de pressão para os diversos números de ondas. 


\subsection{Exemplo 4}

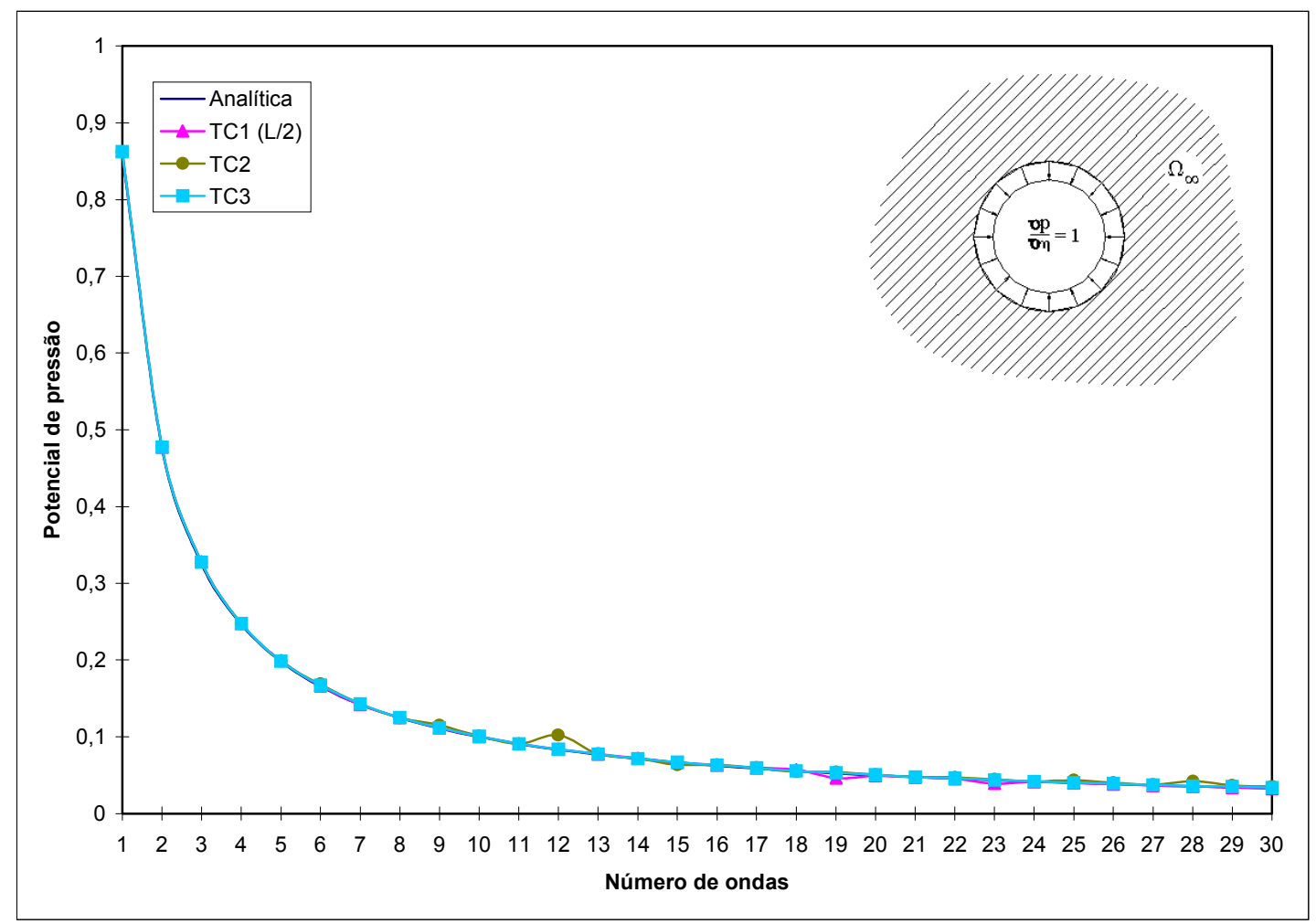

Figura 3.4 - Número de ondas $\times$ potencial de pressão (DL_16)

O contorno é discretizado em 16 elementos lineares, com distribuições lineares de potencial e fluxo de pressão (DL_16).

A resposta obtida neste exemplo foi a melhor entre os já apresentados, as três técnicas utilizadas se aproximaram da solução analítica. Para TC1 houve um pequeno desvio no número de ondas 19 , menor que o desvio notável em $K=15$ do exemplo anterior. $\mathrm{O}$ desvio de resultados de TC3 em relação à solução analítica foi praticamente desprezível. Mesmo TC2 forneceu bons resultados, sendo o desvio no número de ondas 12 o menor entre os exemplos anteriores analisados com a mesma técnica.

\subsection{Exemplo 5}

Nesse exemplo, estuda-se o caso de um cilindro finito, de raio unitário, com pressão constante igual a 10 aplicada no contorno, considerando-se o intervalo de números de ondas de 1 a 10 . O contorno é discretizado em 16 elementos lineares com distribuições constantes 
de potencial e fluxo de pressão (DC_16). As respostas obtidas pelas três técnicas de integração (TC1, TC2 e TC3) são comparadas com os resultados do programa ANSYS

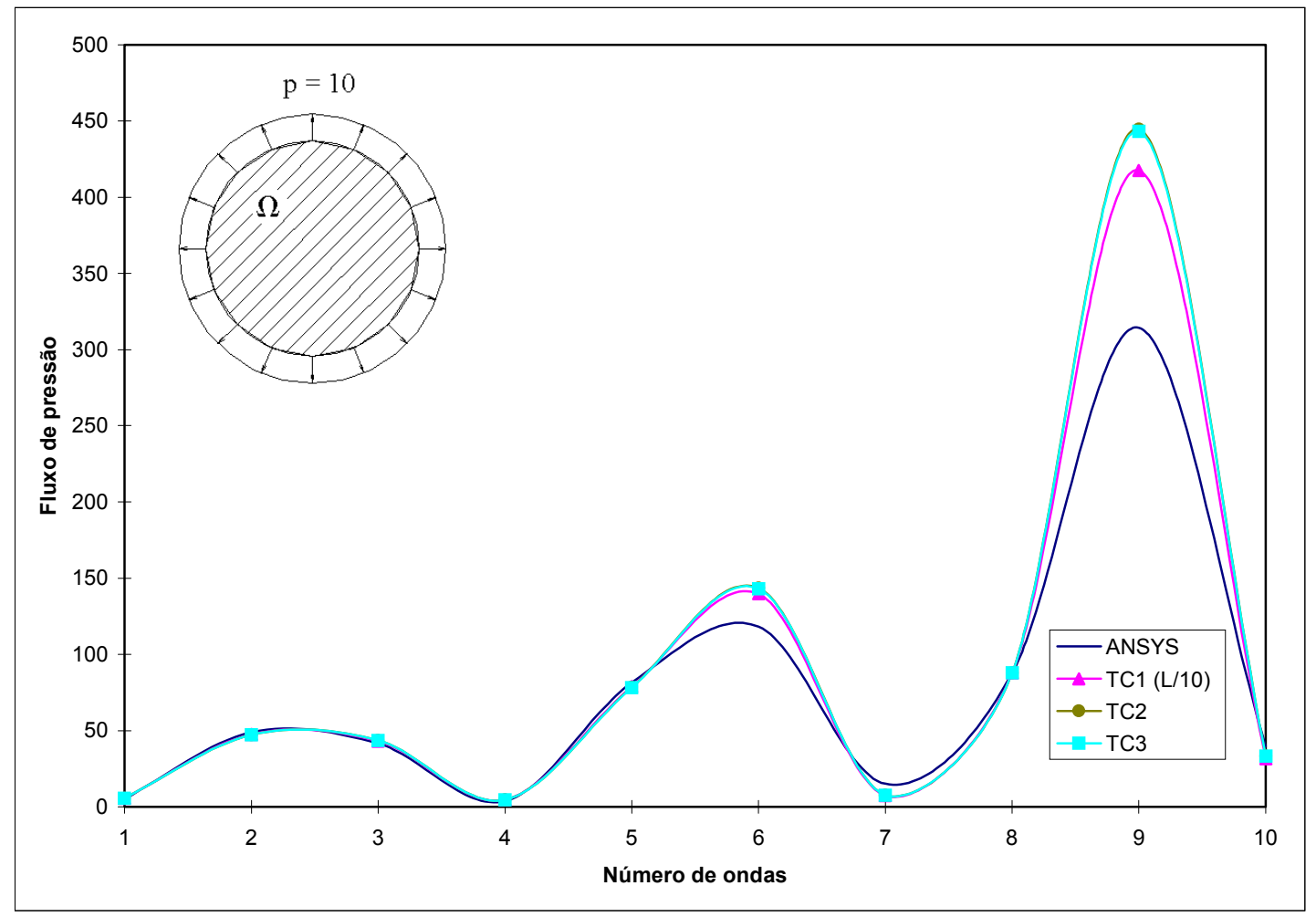

Figura 3.5 - Número de ondas $\times$ fluxo de pressão (DC_16)

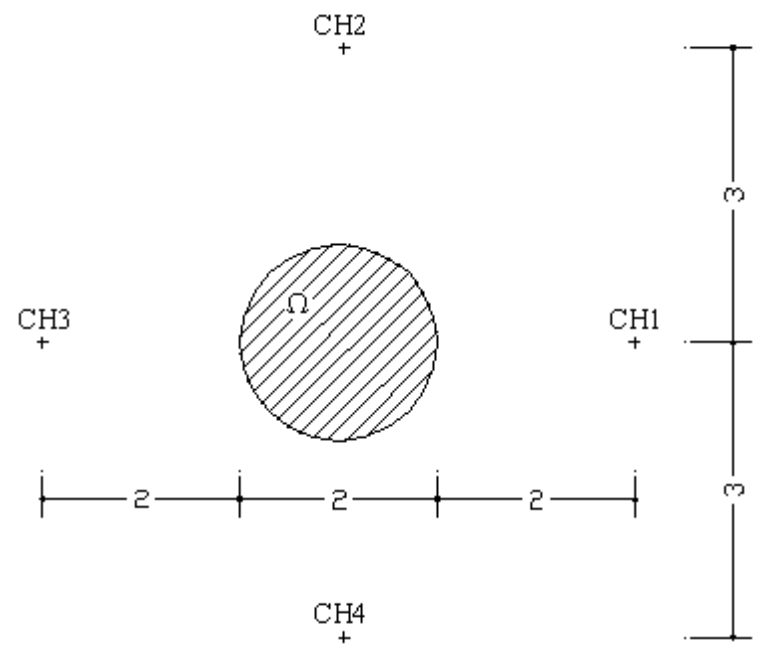

Figura 3.6 - Pontos de CHIEF

\footnotetext{
${ }^{1}$ Foram utilizados 3655 elementos finitos do tipo FLUID29.
} 
Observa-se pelo gráfico da figura 3.5 que existem valores máximos e mínimos reais, relacionados com as freqüências naturais do corpo. A utilização do CHIEF não melhora a resposta em domínios finitos, TC2 e TC3 apresentaram praticamente a mesma resposta, pois neste caso não há problema de não unicidade de resposta. Na figura 3.6 estão ilustrados os 4 pontos de CHIEF utilizados. A resposta de TC1, com distâncias de afastamento dos pontos de colocação iguais a $L / 10$, foi a mais próxima da resposta do ANSYS. Até o número de ondas 5, as respostas do ANSYS e das três técnicas de integração utilizadas foram praticamente iguais.

\subsection{Exemplo 6}

O problema analisado consiste em um domínio finito na forma de quadrado, cujo lado tem dimensão igual a 5; a discretização é feita com 20 elementos lineares de contorno, com distribuições lineares de potencial e fluxo de pressão (DL_20). Para a técnica TC1, a distância de afastamento $d$ utilizada na definição dos pontos de colocação fora do domínio é de $L / 25$. A pressão prescrita no contorno é igual a 3; o número de ondas é igual a 1 . As respostas obtidas pelo MEC são comparadas com as do programa ANSY 2 ? Foram utilizados 4 pontos de CHIEF, ilustrados na figura abaixo.

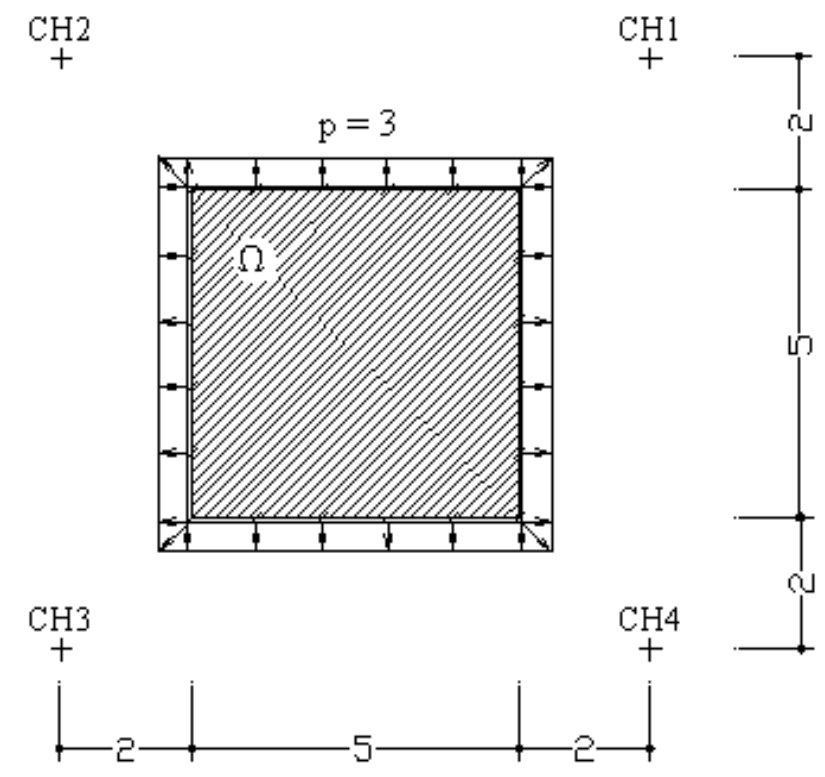

Figura 3.7 - Geometria, condições de contorno e pontos de CHIEF

\footnotetext{
${ }^{2}$ Foram utilizados 10000 elementos finitos do tipo FLUID29.
} 
Também são calculados os potenciais de pressão em pontos internos do domínio. Os pontos internos são apresentados na próxima figura e suas posições e valores em potencial apresentados na tabela 3.3. Através do programa ANSYS, obtiveram-se ainda números de ondas relacionados com as freqüências naturais do problema: $K_{0}=[0.89,1.41,1.78,1.99$, $2.27, \ldots]$.

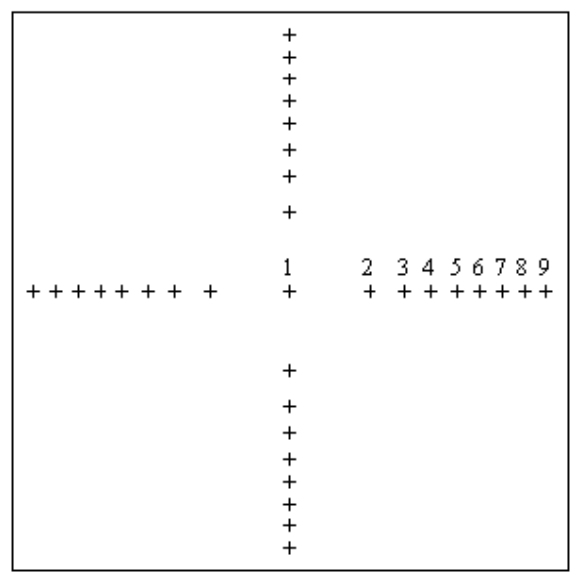

Figura 3.8 - Pontos internos para o cálculo dos potenciais de pressão

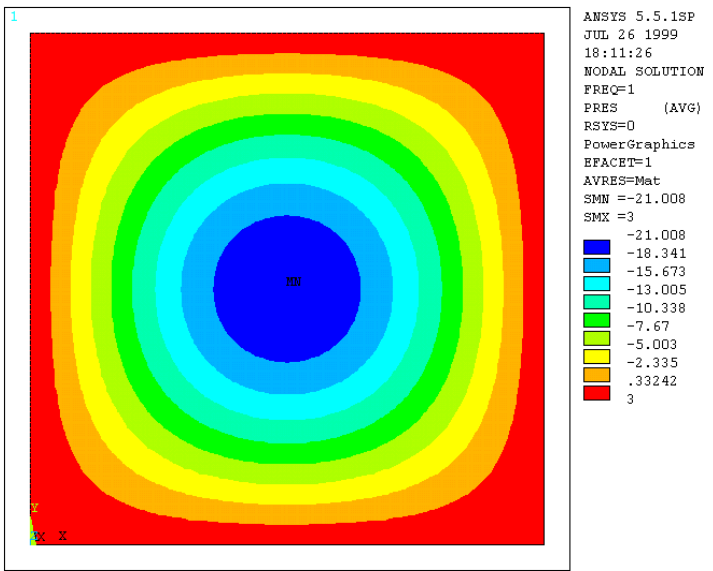

Figura 3.9 - Potenciais de pressão obtidos pelo programa ANSYS

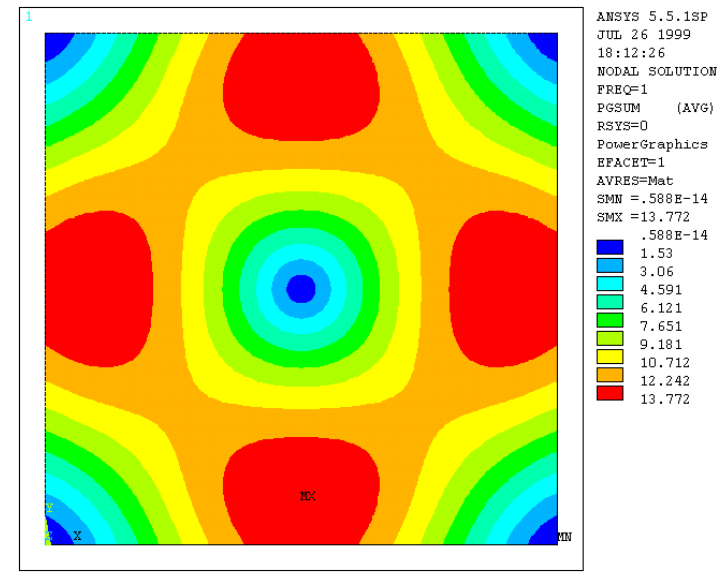

Figura 3.10 - Fluxos de pressão obtidos pelo programa ANSYS

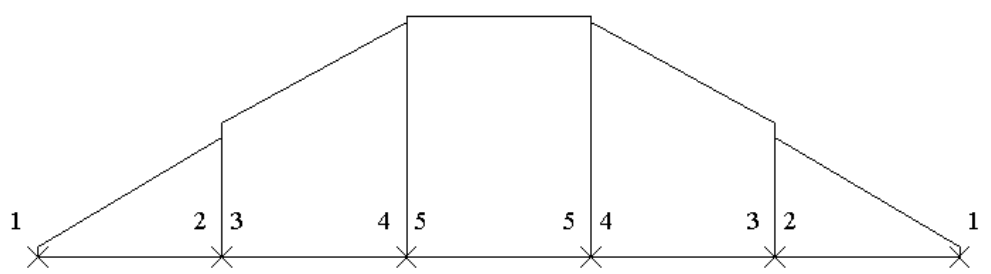

Figura 3.11 - Distribuição de fluxo de pressão nos lados do quadrado (DL_20) 
Tabela 3.2 - Fluxos de pressão nos pontos nodais do quadrado (DL_20)

\begin{tabular}{|c|c|c|c|c||}
\hline NÓ & ANSYS & TC1 (L/25) & TC2 & TC3 \\
\hline 1 & 0.0000 & 0.5427 & 0.5600 & 0.5627 \\
\hline 2 & 6.8860 & 6.5462 & 6.5722 & 6.5741 \\
\hline 3 & 6.8860 & 7.0991 & 7.1113 & 7.1110 \\
\hline 4 & 13.007 & 12.6981 & 12.7360 & 12.7371 \\
\hline 5 & 13.007 & 12.8781 & 12.9118 & 12.9125 \\
\hline
\end{tabular}

Tabela 3.3 - Potenciais de pressão nos pontos internos

\begin{tabular}{|c|c|c|c|c|c||}
\hline PONTO & DIST. & ANSYS & TC1 (L/25) & TC2 & TC3 \\
\hline 1 & 0.000 & -21.0080 & -21.0055 & -21.0542 & -21.0553 \\
\hline 2 & 0.714 & -18.3410 & -18.3929 & -18.4355 & -18.4365 \\
\hline 3 & 1.032 & -15.6730 & -15.7021 & -15.7384 & -15.7393 \\
\hline 4 & 1.270 & -13.0050 & -13.1877 & -13.2181 & -13.2188 \\
\hline 5 & 1.508 & -10.3380 & -10.3386 & -10.3622 & -10.3627 \\
\hline 7 & 1.706 & -7.6700 & -7.7754 & -7.7927 & -7.7931 \\
\hline 8 & 1.905 & -5.0300 & -5.1015 & -5.1123 & -5.1125 \\
\hline 9 & 2.103 & -2.3350 & -2.3765 & -2.3806 & -2.3806 \\
\hline \hline
\end{tabular}

As respostas em fluxo de pressão nos lados do quadrado (tabela 3.2) apresentaram em comum, para todas as técnicas utilizadas, uma tendência de distribuição trapezoidal. As técnicas TC2 e TC3 forneceram praticamente os mesmos resultados, sendo ligeiramente melhores que os fornecidos pela técnica TC1. As diferenças nodais 2-3 e 4-5 para TC2 e TC3 foram menores que as de TC1; os valores nodais de TC2 e TC3 se aproximaram mais dos obtidos com o programa ANSYS do que os valores de TC1. Paradoxalmente, os potenciais nos pontos internos (tabela 3.3) obtidos por TC1 foram mais próximos dos fornecidos pelo ANSYS do que os de TC2 e TC3, que não apresentaram diferenças

\footnotetext{
${ }^{3}$ Distância a partir do centro de gravidade do quadrado (ponto 1 da figura 3.8).
} 
significativas entre si. No geral, pode-se afirmar que os resultados obtidos pelas três técnicas de integração utilizadas (TC1, TC2 e TC3) foram bons. Para corroborar a afirmação, basta confrontar os resultados obtidos (tabelas 3.2 e 3.3) com o número de elementos utilizados pelo ANSYS (10000) e o pelo MEC (20).

\subsection{Exemplo 7}

O exemplo é constituído de um cilindro de raio unitário, envolto por um meio acústico infinito, com potencial de pressão igual a 10 prescrito no contorno e 4 núcleos de pressão concentrados de valor 50 aplicados no domínio. Os núcleos de pressão estão localizados nos eixos horizontal e vertical, a uma distância de duas unidades do centro do cilindro. As respostas são apresentadas como médias aritméticas dos módulos dos fluxos de pressão no contorno, para os números de ondas de 1 a 10. Para a técnica TC1, a distância de afastamento do ponto de colocação ao elemento é de $L / 20$. O contorno é discretizado em 16 elementos lineares, com distribuições lineares de potencial e fluxo de pressão (DL_16). Para a comparação de resultados é utilizada a solução analítica da equação integral de contorno (2.20) no ponto central do cilindro, que também é utilizado como ponto de CHIEF.

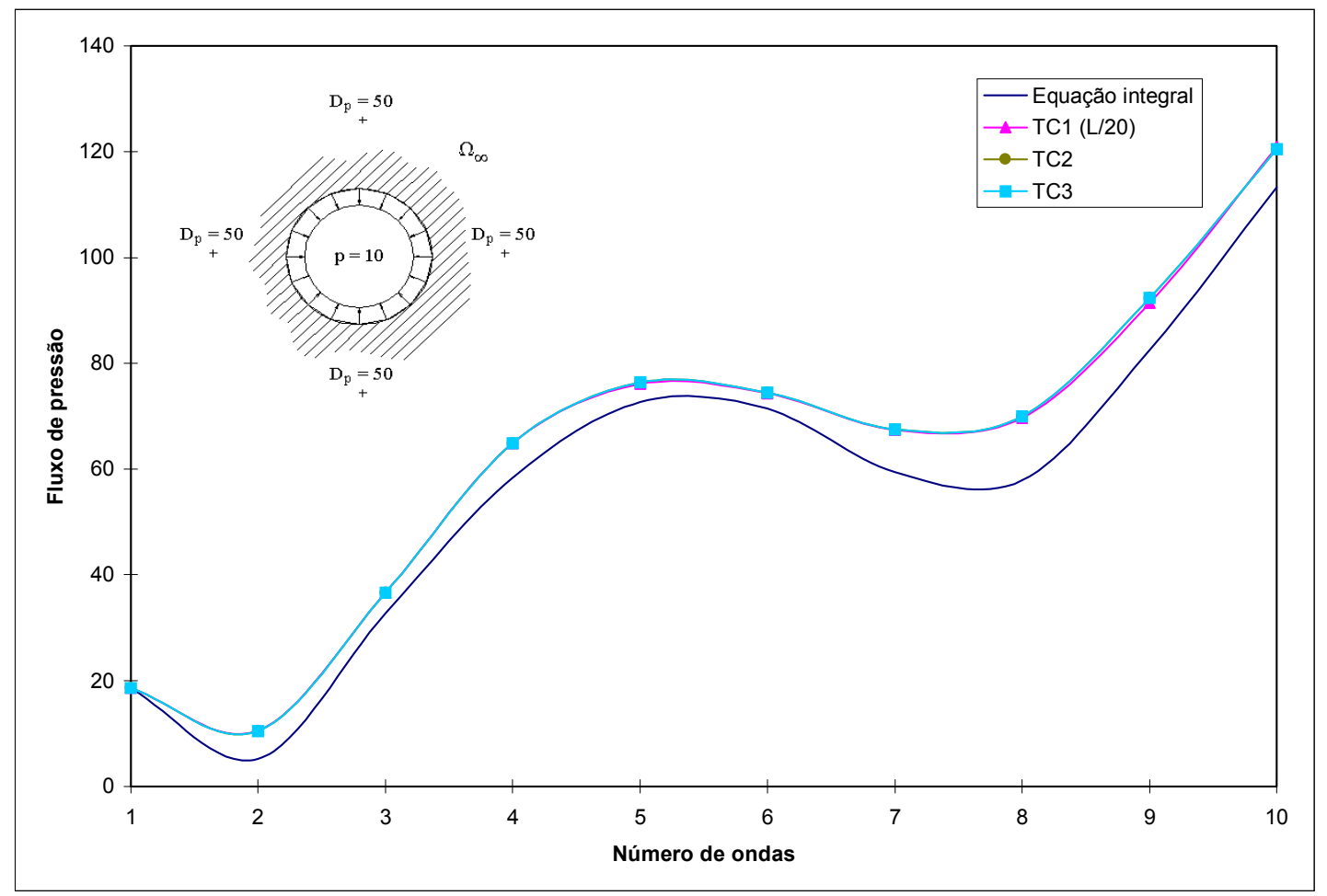

Figura 3.12 - Número de ondas $\times$ fluxo de pressão (DL_16) 
Os resultados obtidos com as três técnicas de integração (TC1, TC2 e TC3) foram praticamente iguais e se aproximaram da solução média da equação integral do problema. É interessante notar que, assim como no caso de domínio finito, na presença de termo de domínio não é necessário aplicar o $\mathrm{CHIEF}$, as respostas de TC2 e TC3 foram bem próximas.

Cabe aqui um comentário sobre unicidade de resposta. O CHIEF é aplicado para resolver o problema da não unicidade de resposta em domínios infinitos. Este problema está relacionado aos autovalores obtidos pela formulação matricial da equação integral de contorno e que não existem no problema físico. Quando existe termo de domínio, diferente de zero, e as variáveis prescritas no contorno são iguais a zero, o problema com os autovalores fictícios desaparece, o sistema matricial não pode mais se tornar homogêneo.

\subsection{Exemplo 8}

Nesse exemplo é estudada a propagação de ondas em um meio acústico semiinfinito. É uma análise especialmente importante no estudo da atenuação de pressão acústica por barreiras. Estuda-se a propagação de uma frente de ondas, gerada por um núcleo de pressão distribuído em linha com valor $10 \mathrm{~Pa} / \mathrm{m}^{2} / \mathrm{m}$, incidente em uma barreira rígida acusticamente. O termo rigidez acústica indica uma impedância infinita à propagação das ondas, ou seja, não há transmissão de ondas através da superfície onde a onda incide, $q=0$.

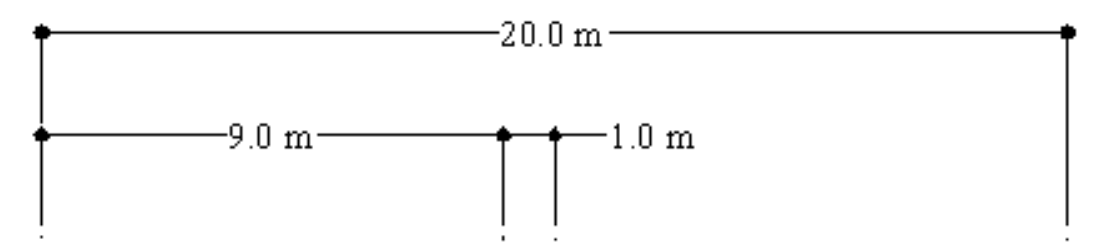

$\Omega_{\infty / 2}$

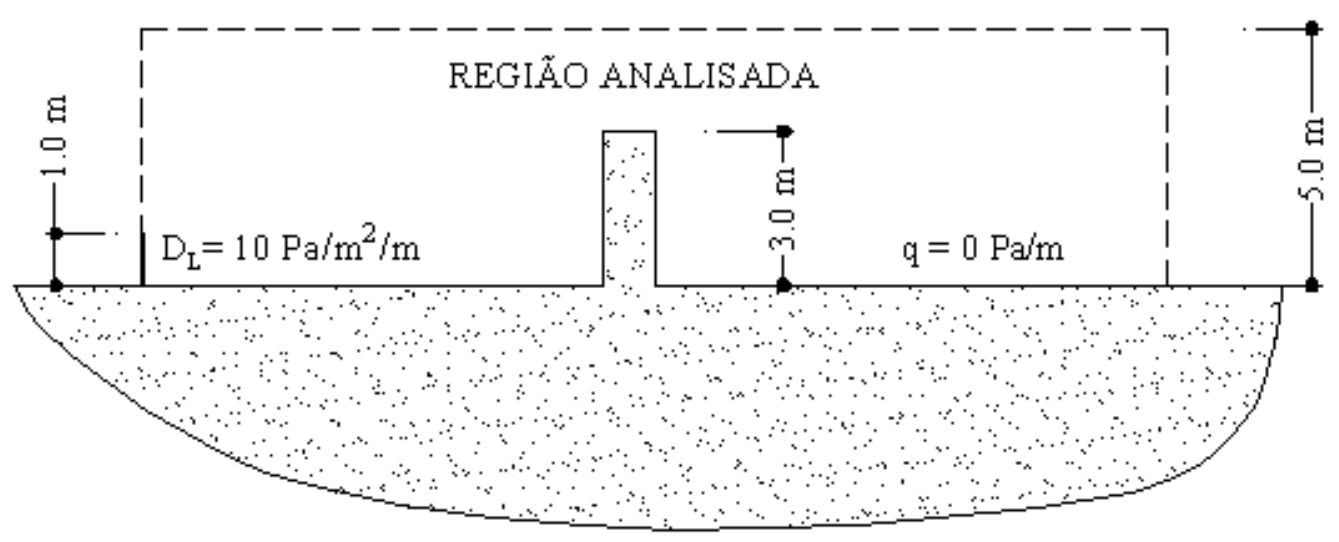

Figura 3.13 - Geometria da barreira acústica no domínio semi-infinito 
A figura 3.13 também indica a posição e dimensão do núcleo de pressão distribuído em linha $D_{L}$. O estudo da propagação da frente de ondas é feito em uma região determinada de $20 \times 5 \mathrm{~m}^{2}$, ilustrada na mesma figura, para uma freqüência de $15 \mathrm{~Hz}$ (ciclos por segundo).

O meio de propagação de ondas é o ar, a uma temperatura de $20^{\circ} \mathrm{C}$, portanto, a velocidade de propagação de ondas vale $c=343 \mathrm{~m} / \mathrm{s}$. O número de ondas é calculado com a freqüência angular:

$$
K=\frac{\omega}{c}=\frac{2 \pi \cdot 15}{343}=0.2747748676609 \mathrm{~m}^{-1}
$$

A simulação do domínio semi-infinito é feita utilizando-se um domínio infinito, onde as dimensões da região analisada sejam pequenas em comparação com a extensão do contorno.

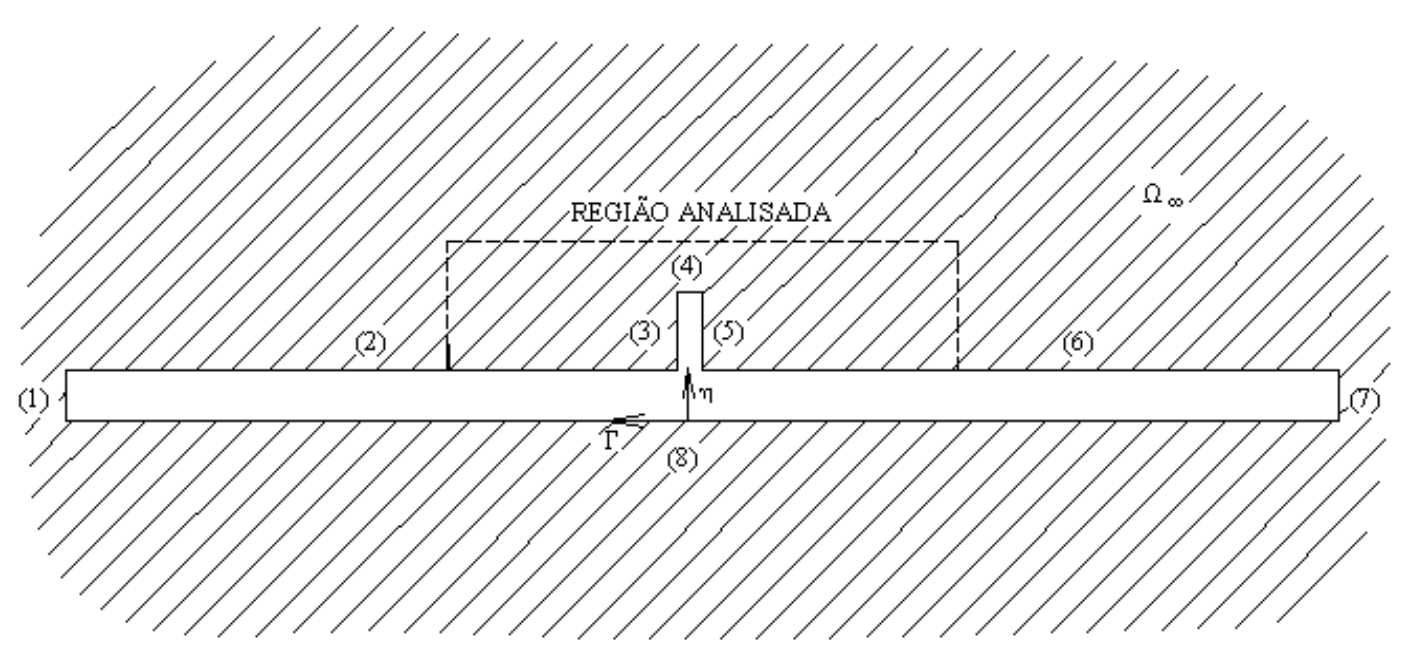

Figura 3.14 - Simulação do domínio semi-infinito

São utilizadas distribuições lineares de potencial e fluxo. Dentro da região analisada são tomados 110 pontos internos para o cálculo de potenciais de pressão.

Foram testadas várias dimensões para o contorno e são apresentados como exemplos dois casos onde consideráveis aumentos na geometria produziram pequenas variações nos potenciais dos pontos internos analisados, localmente a resposta convergiu. As duas malhas de contorno analisadas foram resolvidas pela técnica de integração TC1, com uma distância de afastamento igual a $L / 20$. O número total de elementos de contorno na malha é definido, de acordo com a figura 3.14, pela soma dos elementos de 8 trechos distintos. 
COTAS EM $[\mathrm{m}]$

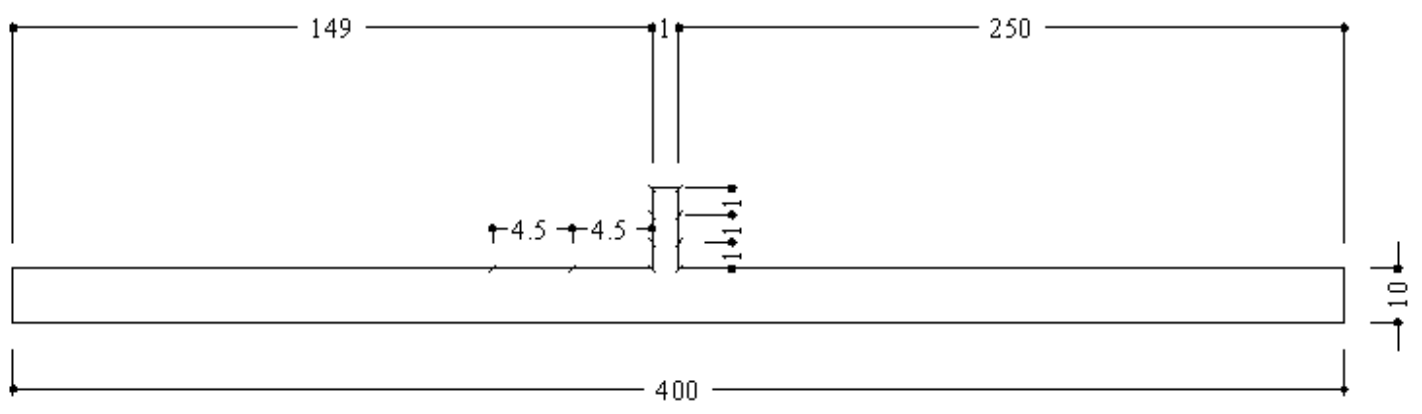

Figura 3.15 - Geometria da primeira malha (DL_90)

Configuração dos trechos na malha DL_90:

(1) e (7) - 1 elemento de tamanho $10 \mathrm{~m}$ por trecho

(2) - 16 elementos: 14 de tamanho $10 m$ e 2 de tamanho $4.5 m$

(3) e (5) - 3 elementos de tamanho $1 \mathrm{~m}$ por trecho

(4) - 1 elementos de tamanho $1 \mathrm{~m}$

(6) - 25 elementos de tamanho $10 \mathrm{~m}$

(8) - 40 elementos de tamanho $10 \mathrm{~m}$

COTAS EM [m]

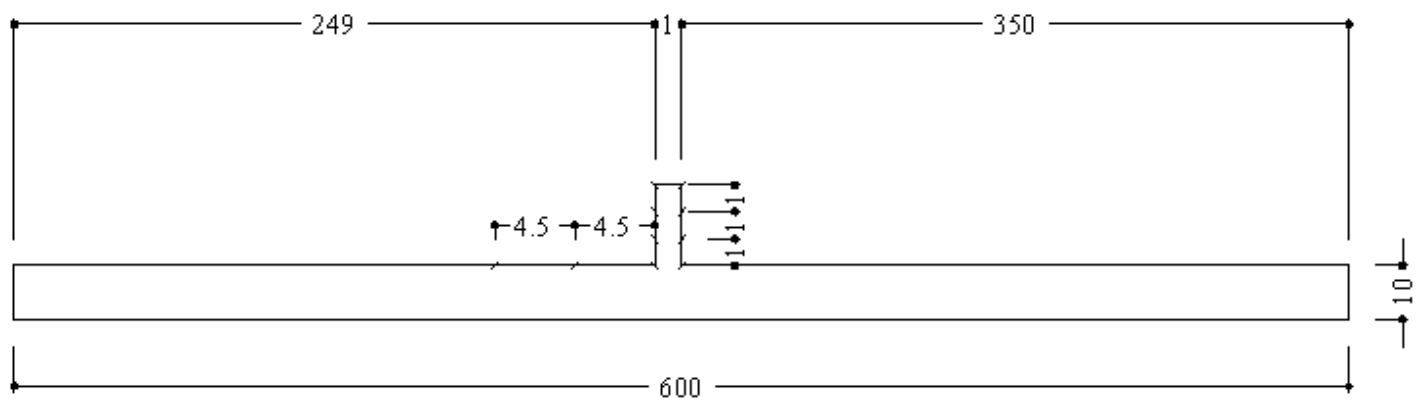

Figura 3.16 - Geometria da segunda malha (DL_130)

Configuração dos trechos na malha DL_130:

(1) e (7) - 1 elemento de tamanho $10 m$ por trecho

(2) - 26 elementos: 24 de tamanho $10 \mathrm{~m}$ e 2 de tamanho $4.5 \mathrm{~m}$

(3) e (5) - 3 elementos de tamanho $1 \mathrm{~m}$ por trecho

(4) - 1 elementos de tamanho $1 \mathrm{~m}$

(6) - 35 elementos de tamanho $10 \mathrm{~m}$

(8) - 60 elementos de tamanho $10 \mathrm{~m}$ 
Os resultados dos potenciais de pressão nos pontos interno ao domínio, localizados na parte abaixo do contorno para as malhas DL_90 e DL_130, se aproximaram de zero. Os potenciais de pressão nos 110 pontos localizados na região de interesse são apresentados na forma de curvas de isopressão. Para enriquecer a análise, é apresentado o resultado de um problema com malha semelhante a DL_130, sem a barreira acústica, também utilizando a técnica de integração TC1 com $L / 20$.

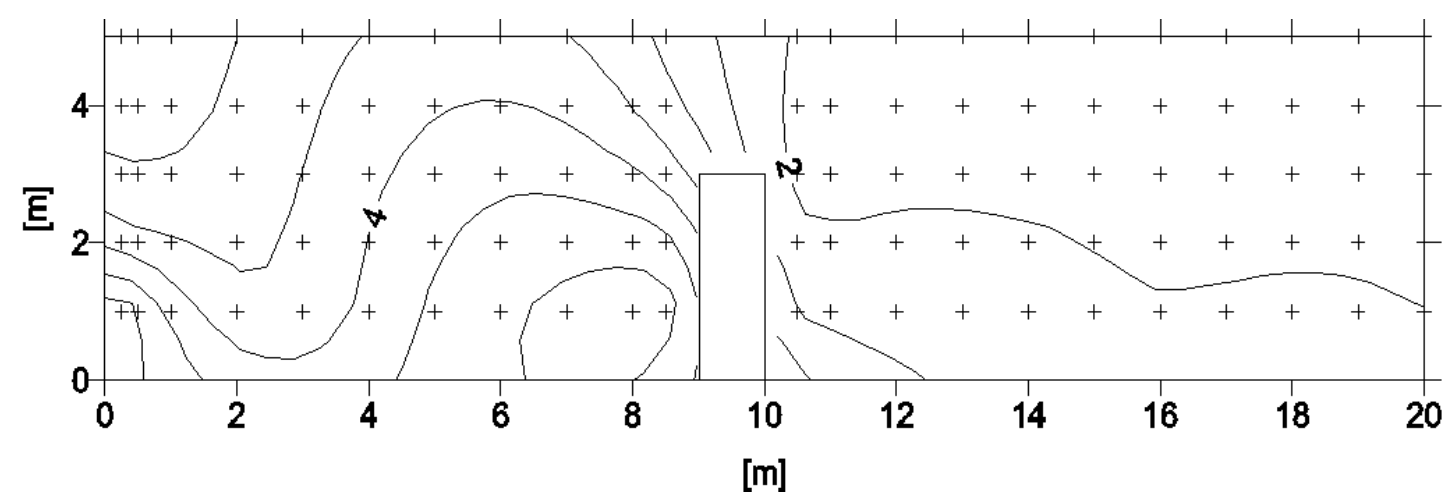

Figura 3.17 - Pressões acústicas próximas à barreira no caso da malha DL_90

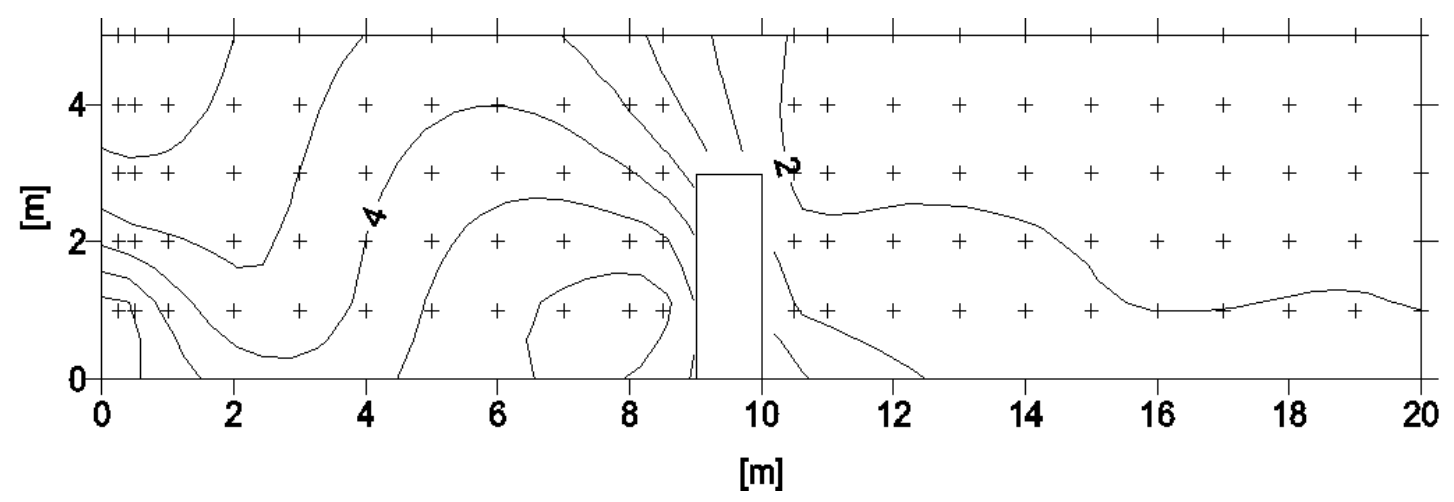

Figura 3.18 - Pressões acústicas próximas à barreira no caso da malha DL_130



Figura 3.19 - Pressões acústicas na região apresentada nas figuras anteriores, sem a barreira 


\section{O MÉTODO DOS ELEMENTOS DE CONTORNO APLICADO A PROBLEMAS ELASTODINÂMICOS BIDIMENSIONAIS NO DOMÍNIO DA FREQÜÊNCIA}

\subsection{Relações básicas}

As relações descritas neste capítulo serão apresentadas na forma indicial (Anexo D). Como o problema estudado é bidimensional, os índices utilizados $(l, m, k)$ terão valores iguais a 1 ou 2, representando as direções $\mathrm{X}$ e $\mathrm{Y}$ respectivamente.

Inicialmente as equações serão desenvolvidas admitindo-se estado plano de deformações (EPD) ${ }^{1}$. Analisando-se um ponto qualquer do sólido, pode-se representar esquematicamente as componentes de deformação $\left(\varepsilon_{m k}\right)$ como indicado na figura abaixo.

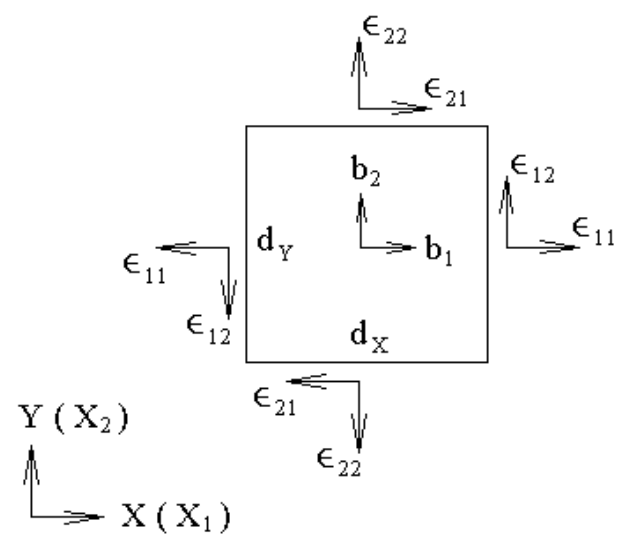

Figura 4.1 - EPD em um ponto no plano do sólido

$\mathrm{Na}$ figura 4.1 o ponto é representado por um quadrado de lados infinitesimais, com faces paralelas aos eixos cartesianos; $b_{1}$ e $b_{2}$ são possíveis forças de corpo presentes no sólido.

\footnotetext{
${ }^{1}$ Situação em que uma das dimensões do sólido é muito maior que as outras duas.
} 
Outras hipóteses para o desenvolvimento das equações são de sólido isótropo homogêneo com comportamento linear ${ }^{2}$.

As deformações são representadas por um tensor de segunda ordem simétrico $\left(\varepsilon_{m k}=\varepsilon_{k m}\right)$, dependente das componentes de deslocamento $\left(u_{1}\right.$ e $\left.u_{2}\right)$. Para o caso de pequenas deformações, tem-se:

$$
\varepsilon_{m k}=\frac{1}{2} \cdot\left(u_{m, k}+u_{k, m}\right)
$$

Através do balanço do momento linear obtém-se a equação de equilíbrio escrita em função das componentes de tensão $\left(\sigma_{m k}\right)$.

$$
\sigma_{m k, k}+\rho \cdot b_{m}=\rho \cdot \ddot{u}_{m} \quad \text { (equação de equilíbrio) }
$$

Na equação (4.2), $\ddot{u}_{m}$ representa as componentes de aceleração no ponto analisado e $\rho$ a densidade do sólido.

A lei de Hooke generalizada relaciona as componentes de tensão e deformação através de duas constantes do material, o módulo de elasticidade longitudinal $(E)$ e o coeficiente de Poisson $(v)$. As constantes $E$ e $v$ podem ser expressas através das constantes de Lamé $\lambda$ e $\mu$. No caso de EPD, a lei de Hooke pode ser expressa por:

$$
\sigma_{m k}=\lambda \cdot \varepsilon_{l l} \cdot \delta_{m k}+2 \cdot \mu \cdot \varepsilon_{m k} \quad \text { (lei de Hooke) }
$$

As constantes de Lamé estão relacionadas com as constantes clássicas pelas seguintes expressões:

$$
\begin{gathered}
\mu=\frac{E}{2 \cdot(1+v)} \\
\lambda=\frac{2 \cdot \mu \cdot v}{1-2 \cdot v}
\end{gathered}
$$

Onde:

$\mu$ é o módulo de elasticidade transversal ${ }^{[}$.

A equação (4.3) é utilizada para EPD. Pode-se desenvolver uma equação análoga para o estado plano de tensões (EPT) ${ }^{\frac{1}{4}}$. Basta manter o mesmo $\mu$, calcular um coeficiente de Poisson equivalente $(\bar{v})$ e um novo $\lambda$.

$$
\bar{v}=\frac{v}{1+v}
$$

\footnotetext{
${ }^{2}$ As expressões que relacionam as componentes de tensão e deformação são admitidas como lineares.

${ }^{3}$ Também representado pela letra $G$ em vários trabalhos.

${ }^{4}$ Situação em que uma das dimensões do sólido é bem menor do que as outras duas.
} 


$$
\lambda=\frac{2 \cdot \mu \cdot \bar{v}}{1-2 \cdot \bar{v}}
$$

Substituindo-se a equação (4.3) em (4.2), tem-se:

$$
\begin{gathered}
\frac{\partial}{\partial x_{k}}\left(\lambda \cdot \varepsilon_{l l} \cdot \delta_{m k}+2 \cdot \mu \cdot \varepsilon_{m k}\right)+\rho \cdot b_{m}=\rho \cdot \ddot{u}_{m} \\
\frac{\partial}{\partial x_{k}}\left[\lambda \cdot \frac{1}{2} \cdot\left(u_{l, l}+u_{l, l}\right) \cdot \delta_{m k}+2 \cdot \mu \cdot \frac{1}{2} \cdot\left(u_{m, k}+u_{k, m}\right)\right]+\rho \cdot b_{m}=\rho \cdot \ddot{u}_{m} \\
\frac{\partial}{\partial x_{k}}\left[\lambda \cdot u_{l, l} \cdot \delta_{m k}+\mu \cdot u_{m, k}+\mu \cdot u_{k, m}\right]+\rho \cdot b_{m}=\rho \cdot \ddot{u}_{m} \\
\lambda \cdot u_{l, l k} \cdot \delta_{m k}+\mu \cdot u_{m, k k}+\mu \cdot u_{k, m k}+\rho \cdot b_{m}=\rho \cdot \ddot{u}_{m}
\end{gathered}
$$

$\mathrm{Na}$ equação (4.7), $m$ é índice livre e $k$ e $l$ são mudos. Portanto é possível escrever: $u_{l, l k} \cdot \delta_{m k}=u_{l, l m}=u_{k, k m}=u_{k, m k}$. Obtém-se assim uma equação de equilíbrio dinâmico análoga à equação de Navier (caso estático).

$$
\mu \cdot u_{m, k k}+(\lambda+\mu) \cdot u_{k, m k}+\rho \cdot b_{m}=\rho \cdot \ddot{u}_{m}
$$

Pode-se fazer a representação da equação (4.7) na forma vetorial:

$$
\mu \cdot \nabla^{2} \vec{u}+(\lambda+\mu) \cdot \nabla(\nabla \cdot \vec{u})+\rho \cdot \vec{b}=\rho \cdot \ddot{\vec{u}}
$$

A equação de acima descreve o comportamento elastodinâmico de um sólido homogêneo e isótropo em todo o domínio $\Omega$.

Considerando-se comportamento harmônico:

$$
\begin{aligned}
& \vec{u}(t)=\vec{u} \cdot e^{-i \cdot \omega \cdot t} \\
& \vec{b}(t)=\vec{b} \cdot e^{-i \cdot \omega \cdot t}
\end{aligned}
$$

Pode-se desenvolver a equação (4.8).

$$
\begin{gathered}
{\left[\mu \cdot \nabla^{2} \vec{u}+(\lambda+\mu) \cdot \nabla(\nabla \cdot \vec{u})+\rho \cdot \vec{b}\right] \cdot e^{-i \cdot \omega \cdot t}=\rho \cdot \frac{\partial^{2}}{\partial t^{2}}\left(\vec{u} \cdot e^{-i \cdot \omega \cdot t}\right)} \\
{\left[\mu \cdot \nabla^{2} \vec{u}+(\lambda+\mu) \cdot \nabla(\nabla \cdot \vec{u})+\rho \cdot \vec{b}\right] \cdot e^{-i \cdot \omega \cdot t}=\rho \cdot \frac{\partial}{\partial t}\left(\vec{u} \cdot e^{-i \cdot \omega \cdot t}\right) \cdot-i \cdot \omega} \\
\left.\mid \mu \cdot \nabla^{2} \vec{u}+(\lambda+\mu) \cdot \nabla(\nabla \cdot \vec{u})+\rho \cdot \vec{b}\right] \cdot e^{-i \cdot \omega \cdot t}=\rho \cdot \vec{u} \cdot e^{-i \cdot \omega \cdot t} \cdot(-i \cdot \omega)^{2} \\
\mu \cdot \nabla^{2} \vec{u}+(\lambda+\mu) \cdot \nabla(\nabla \cdot \vec{u})+\rho \cdot \vec{b}=-\rho \cdot \omega^{2} \cdot \vec{u}
\end{gathered}
$$

Também é possível representar a equação (4.10) em forma indicial:

$$
\mu \cdot u_{m, k k}+(\lambda+\mu) \cdot u_{k, m k}+\rho \cdot b_{m}=-\rho \cdot \omega^{2} \cdot u_{m}
$$


Uma outra relação fundamental da Teoria da Elasticidade, útil na formulação do MEC aplicado a problemas elastodinâmicos, é a fórmula de Cauchy, que relaciona forças de superfície $\left(p_{m}\right)$ com as componentes de tensão $\left(\sigma_{m k}\right)$ em um ponto do sólido.

$$
p_{m}=\sigma_{m k} \cdot \eta_{k} \quad \text { (fórmula de Cauchy) }
$$

\subsection{Equação integral de contorno}

O equacionamento do MEC para problemas elastodinâmicos no domínio da freqüência parte do teorema da reciprocidade de Betti.

Teorema de Betti: em um sólido de comportamento linear, o trabalho realizado por um conjunto de forças através dos deslocamentos produzidos por um segundo conjunto de forças é igual ao trabalho do segundo conjunto de forças através dos deslocamentos do primeiro conjunto. Sendo as forças consideradas como de superfície (em equilíbrio) e de corpo.

$$
\int_{\Gamma} p \cdot u^{*} d_{\Gamma}+\int_{\Omega} \rho \cdot b \cdot u^{*} d_{\Omega}=\int_{\Gamma} p^{*} \cdot u d_{\Gamma}+\int_{\Omega} \rho \cdot b^{*} \cdot u d_{\Omega} \quad \text { (teorema de Betti) }
$$

A solução fundamental $\left(u_{l m}^{*}\right)$ deve satisfazer a equação (4.11), utilizada para problemas harmônicos. Fisicamente, $u_{l m}^{*}$ representa o efeito nos deslocamentos em um ponto $S$, devido a uma carga concentrada unitária e harmônica aplicada na direção $l$ em um ponto $P$ de um domínio infinito.

Portanto, de (4.14) tem-se: $\rho \cdot b^{*}=\delta_{(S, P)} \cdot \delta_{l m}$.

Pode-se reescrever a equação (4.13) em função das componentes de deslocamentos e forças de superfície para dois conjuntos: o problema físico e a solução fundamental.

$$
\begin{gathered}
\int_{\Gamma} p_{m} \cdot u_{l m}^{*} d_{\Gamma}+\int_{\Omega} \rho \cdot b_{m} \cdot u_{l m}^{*} d_{\Omega}=\int_{\Gamma} p_{l m}^{*} \cdot u_{m} d_{\Gamma}+\int_{\Omega} \delta_{(S, P)} \cdot \delta_{l m} \cdot u_{m} d_{\Omega} \\
c_{(S)} \cdot u_{l(S)}+\int_{\Gamma} p_{l m}^{*} \cdot u_{m} d_{\Gamma}=\int_{\Gamma} u_{l m}^{*} \cdot p_{m} d_{\Gamma}+\int_{\Omega} u_{l m}^{*} \cdot \rho \cdot b_{m} d_{\Omega}
\end{gathered}
$$

(equação integral de contorno)

Algumas simplificação serão feitas neste trabalho. A primeira considera a não existência de forças de corpo nos problemas, obtendo-se a identidade de Somigliana, expressa na seguinte forma:

$$
c_{(S)} \cdot u_{l(S)}+\int_{\Gamma} p_{l m}^{*} \cdot u_{m} d_{\Gamma}=\int_{\Gamma} u_{l m}^{*} \cdot p_{m} d_{\Gamma} \quad \text { (identidade de Somigliana) }
$$


A segunda simplificação é a obtenção do sistema de equações a partir da escolha de pontos de colocação apenas fora do domínio, $c_{(S)}=0$; em geral esta será a estratégia utilizada. Ou seja, será utilizada uma técnica de integração com ponto de colocação quase singular, análoga à apresentada no item 2.4.2, com utilização de sub-elementação.

$$
\int_{\Gamma} p_{l m}^{*} \cdot u_{m} d_{\Gamma}=\int_{\Gamma} u_{l m}^{*} \cdot p_{m} d_{\Gamma} \quad \text { (equação integral de contorno utilizada) }
$$

As expressões de $u_{l m}^{*}$ e $p_{l m}^{*}$ podem ser encontradas em DOMINGUEZ (1993).

$$
u_{l m}^{*}=\frac{1}{2 \cdot \pi \cdot \rho \cdot c_{2}^{2}} \cdot\left(\psi \cdot \delta_{l m}-\chi \cdot R, \cdot R,_{m}\right)
$$

Onde:

$$
\begin{gathered}
\psi=K_{0}\left(\frac{i \cdot \omega \cdot R}{c_{2}}\right)-i \cdot \frac{c_{2}}{\omega \cdot R} \cdot\left[K_{1}\left(\frac{i \cdot \omega \cdot R}{c_{2}}\right)-\frac{c_{2}}{c_{1}} \cdot K_{1}\left(\frac{i \cdot \omega \cdot R}{c_{1}}\right)\right] \\
\chi=K_{2}\left(\frac{i \cdot \omega \cdot R}{c_{2}}\right)-\frac{c_{2}^{2}}{c_{1}^{2}} \cdot K_{2}\left(\frac{i \cdot \omega \cdot R}{c_{1}}\right) \\
c_{1}=\sqrt{\frac{\lambda+2 \cdot \mu}{\rho}} \\
c_{2}=\sqrt{\frac{\mu}{\rho}}
\end{gathered}
$$

$R$ é a distância entre os pontos $S$ e $P$.

$K_{0}, K_{1}$ e $K_{2}$ são funções modificadas de Bessel de segunda classe e ordens 0,1 e 2 respectivamente.

$$
\begin{aligned}
p_{l m}^{*} & =\frac{1}{2 \cdot \pi} \cdot\left[\left(\frac{d_{\psi}}{d_{R}}-\frac{1}{R} \cdot \chi\right) \cdot\left(\delta_{l m} \cdot \frac{\partial R}{\partial \eta}+R,_{m} \cdot \eta_{l}\right)-\frac{2}{R} \cdot \chi \cdot\left(\eta_{m} \cdot R,_{l}-2 \cdot R,,_{l} \cdot R,,_{m} \cdot \frac{\partial R}{\partial \eta}\right)\right. \\
& \left.-2 \cdot \frac{d_{\chi}}{d_{R}} \cdot R,,_{l} \cdot R,,_{m} \cdot \frac{\partial R}{\partial \eta}+\left(\frac{c_{1}^{2}}{c_{2}^{2}}-2\right) \cdot\left(\frac{d_{\psi}}{d_{R}}-\frac{d_{\chi}}{d_{R}}-\frac{1}{R} \cdot \chi\right) \cdot R,{ }_{l} \cdot \eta_{m}\right]
\end{aligned}
$$

Onde:

$$
\begin{aligned}
\frac{d_{\psi}}{d_{R}} & =i \cdot \omega \cdot K_{1}\left(\frac{i \cdot \omega \cdot R}{c_{2}}\right) \cdot\left[\frac{2 \cdot c_{2}}{\omega^{2} \cdot R^{2}}-\frac{1}{c_{2}}\right]-i \cdot K_{1}\left(\frac{i \cdot \omega \cdot R}{c_{1}}\right) \cdot \frac{c_{2}^{2}}{c_{1} \cdot \omega} \cdot \frac{2}{R^{2}} \\
& -K_{0}\left(\frac{i \cdot \omega \cdot R}{c_{2}}\right) \cdot \frac{1}{R}+K_{0}\left(\frac{i \cdot \omega \cdot R}{c_{1}}\right) \cdot \frac{c_{2}^{2}}{c_{1}^{2}} \cdot \frac{1}{R}
\end{aligned}
$$




$$
\begin{aligned}
\frac{d_{\chi}}{d_{R}} & =-K_{1}\left(\frac{i \cdot \omega \cdot R}{c_{2}}\right) \cdot \frac{i \cdot \omega}{c_{2}}-\frac{2}{R} \cdot K_{2}\left(\frac{i \cdot \omega \cdot R}{c_{2}}\right)+\frac{c_{2}^{2}}{c_{1}^{2}} \cdot \frac{i \cdot \omega}{c_{1}} \cdot K_{1}\left(\frac{i \cdot \omega \cdot R}{c_{1}}\right) \\
& +\frac{2}{R} \cdot \frac{c_{2}^{2}}{c_{1}^{2}} \cdot K_{2}\left(\frac{i \cdot \omega \cdot R}{c_{1}}\right)
\end{aligned}
$$

\subsection{Representação matricial}

Neste trabalho, as variáveis de contorno, deslocamento $\left(u_{m}\right)$ e força de superfície $\left(p_{m}\right)$, terão distribuições lineares no contorno (conforme exposto no item 2.3.1). Para o caso bidimensional, em cada nó existem dois valores de $u_{m}$ e $p_{m}$, um na direção $X$ e outro na direção $Y$. Portanto, considerando $N N D$ nós, o sistema matricial terá dimensão $N=2 \cdot N N D$. Para cada par nodal $u_{m}$ está relacionada uma matriz $[h]_{2 \times 2}=\int_{\Gamma} p_{l m}^{*} \cdot \phi_{k} d_{\Gamma}$ $(l=1,2$ e $m=1,2)$. Da mesma forma, para cada par nodal $p_{m}$ relaciona-se uma matriz $[g]_{2 \times 2}=\int_{\Gamma} u_{l m}^{*} \cdot \phi_{k} d_{\Gamma}$.

As matrizes $[h]_{2 \times 2}$ e $[g]_{2 \times 2}$ funcionam como se fossem os elementos das matrizes $[H]$ e $[G]$ do problema potencial (capítulo 2); os pares de $u_{m}$ e $p_{m}$ se comportam como as variáveis. A diferença é que um par pode ter um valor prescrito e um incógnito ou dois valores prescritos ou dois valores incógnitos.

A equação integral de contorno (4.17) é escrita matricialmente por:

$$
[H] \cdot\{u\}=[G] \cdot\{p\} \quad \text { (equação matricial de contorno) }
$$

De forma explícita, pode-se representar (4.22) da seguinte maneira:

$$
\left[\begin{array}{cccc}
{[h]_{11}} & {[h]_{12}} & \cdots & {[h]_{1 N}} \\
{[h]_{21}} & {[h]_{22}} & \cdots & {[h]_{2 N}} \\
\vdots & \vdots & \ddots & \vdots \\
{[h]_{N 1}} & {[h]_{N 2}} & \cdots & {[h]_{N N}}
\end{array}\right] \cdot\left\{\begin{array}{c}
\{u\}_{1} \\
\{u\}_{2} \\
\vdots \\
\{u\}_{N}
\end{array}\right\}=\left[\begin{array}{cccc}
{[g]_{11}} & {[g]_{12}} & \cdots & {[g]_{1 N}} \\
{[g]_{21}} & {[g]_{22}} & \cdots & {[g]_{2 N}} \\
\vdots & \vdots & \ddots & \vdots \\
{[g]_{N 1}} & {[g]_{N 2}} & \cdots & {[g]_{N N}}
\end{array}\right] \cdot\left\{\begin{array}{c}
\{p\}_{1} \\
\{p\}_{2} \\
\vdots \\
\{p\}_{N}
\end{array}\right\}
$$

Onde:

$$
[h]=\left[\begin{array}{ll}
h_{11} & h_{12} \\
h_{21} & h_{22}
\end{array}\right],[g]=\left[\begin{array}{ll}
g_{11} & g_{12} \\
g_{21} & g_{22}
\end{array}\right],\{u\}=\left\{\begin{array}{l}
u_{1} \\
u_{2}
\end{array}\right\} ;\{p\}=\left\{\begin{array}{l}
p_{1} \\
p_{2}
\end{array}\right\}
$$


Separando-se os valores prescritos dos incógnitos em lados diferentes da igualdade, de maneira análoga à apresentada no item 2.3.2, obtém-se um sistema final de equações do tipo $[A] \cdot\{X\}=\{F\}$, pronto para ser resolvido.

\subsection{Exemplo numérico}

Como exemplo é analisado o caso de uma chapa elastodinâmica, em EPD, submetida a um carregamento $p=20000 \mathrm{~N} / \mathrm{m}$ em um dos lados. O faixa de freqüências varia de 50 a $500 \mathrm{~Hz}$, em intervalos de 50 . O material da chapa apresenta as seguintes propriedades físicas: $E=19.5 \times 10^{10} \mathrm{~N} / \mathrm{m}^{2}, v=0.28 ; \rho=7700 \mathrm{Kg} / \mathrm{m}^{3}$.

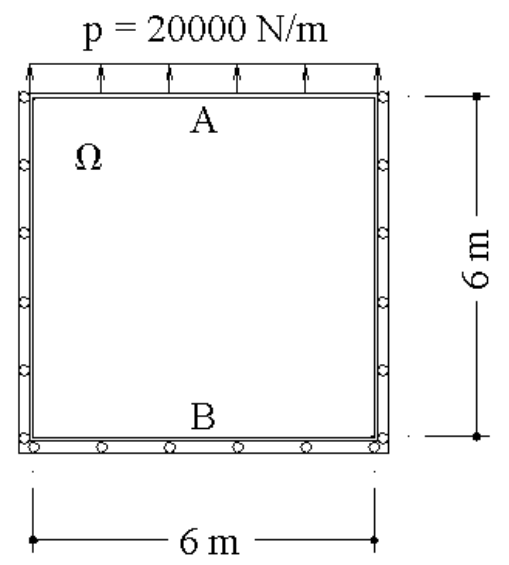

Figura 4.2 - Geometria e condições de contorno do exemplo elastodinâmico

A discretização utilizada no MEC é constituída de 16 elementos lineares de contorno, de tamanhos $L=1.5 \mathrm{~m}$, com distribuições lineares das variáveis de contorno (DL_16). A técnica de integração utilizada é TC1 com distância de afastamento dos pontos de colocação igual a $L / 20$. Os resultados médios nas faces $A$ e $B$ são comparados com os obtidos pelo programa ANSYS, com um malha de $60 \times 60$ elementos finitos. Através do programa ANSYS, obtiveram-se ainda algumas freqüências naturais do problema: $\omega_{0}=[237.09,265.80,442.90,487.55,506.05,612.87,650.67, \ldots] \mathrm{Hz}$. Destacam-se as quatro primeiras freqüências naturais, compreendidas na faixa de freqüências analisadas. 
Tabela 4.1 - Deslocamentos em Y na face A [ $\times 0.0000001 \mathrm{~m}]$

\begin{tabular}{||c|c|c|c||}
\hline FREQÜÊNCIA $[\mathrm{Hz}]$ & ANSYS & TC1 (L/20) & ERRO RELATIVO (\%) \\
\hline 50 & 5.000 & 5.000 & 0 \\
\hline 100 & 5.670 & 5.670 & 0 \\
\hline 150 & 7.440 & 7.440 & 0 \\
\hline 200 & 14.500 & 14.500 & 3 \\
\hline 250 & -34.000 & -33.900 & 5 \\
\hline 300 & -5.320 & -5.470 & 6 \\
\hline 350 & -2.370 & -2.240 & 4 \\
\hline 400 & -0.916 & -0.972 & 0 \\
\hline 450 & -0.273 & -0.261 & 0.251 \\
\hline 500 & 0.251 & & 0 \\
\hline
\end{tabular}

Tabela 4.2 - Reações de apoio em Y na face B [N/m]

\begin{tabular}{||c|c|c|c||}
\hline \hline FREQÜEENCIA [Hz] & ANSYS & TC1 (L/20) & ERRO RELATIVO (\%) \\
\hline 50 & -21150 & -21150 & 0 \\
\hline 100 & -25367 & -25367 & 0 \\
\hline 150 & -36664 & -36665 & 0 \\
\hline 200 & -82223 & -82222 & 0 \\
\hline 250 & 234070 & 234114 & 0 \\
\hline 300 & 49404 & 49408 & 0 \\
\hline 350 & 29404 & 29406 & 0 \\
\hline 400 & 22686 & 22687 & 0 \\
\hline 450 & 20260 & 20260 & 20295 \\
\hline 500 & 20295 & & 0 \\
\hline
\end{tabular}




\section{ACOPLAMENTO FLUIDO-ESTRUTURA (MEC-MEC)}

\subsection{Introdução}

O acoplament 1 entre o fluido e a estrutura, apresentado neste trabalho, envolve domínios de naturezas diferentes, fluidos compressíveis (gases ou líquidos) e sólidos elásticos. Algumas das variáveis do problema na região de interface são comuns aos dois domínios, que são descritos por fenômenos físicos diferentes. O MEC é ideal para análise de domínios infinitos ou semi-infinitos, caso de fluido não confinado.

Existem várias referências de modelos numéricos combinando o MEC e o Método dos Elementos Finitos (MEF) 2 em problemas de acoplamento fluido-estrutura. WILTON (1978), LEWIS et al. (1984) e MORAND et al. (1995) estão entre as referências consultadas mais importantes.

O presente trabalho propõe uma formulação totalmente baseada no MEC para o problema acoplado, apresentando no final deste capítulo um exemplo de chapa elastodinâmica acoplada com um meio fluido compressível (acústico) infinito. Este tipo de análise é especialmente importante em estudos de Acústica e Vibrações em sistemas mecânicos compostos por fluidos e estruturas acoplados. A chapa e o meio acústico são considerados materiais isótropos e homogêneos.

\subsection{Interação entre o meio acústico e a estrutura}

$\mathrm{O}$ acoplamento fluido-estrutura é feito em função das variáveis pressão $(p) \mathrm{e}$ deslocamento $(u)$ na direção normal $(\eta)$ à interface entre os dois meios. Portanto, é necessário rescrever as equações a serem acopladas em função destas variáveis.

\footnotetext{
${ }^{1}$ Também chamado de interação.

${ }^{2}$ Utilizado na modelagem numérica do domínio da estrutura.
} 
Para o fluido, toma-se a equação (1.24) e admite-se comportamento harmônico: $p=p \cdot e^{-i \cdot \omega \cdot t}$ e $\phi=\phi \cdot e^{-i \cdot \omega \cdot t}$.

$$
\begin{gathered}
p=-\rho_{0} \cdot \frac{\partial \phi}{\partial t} \\
p \cdot e^{-i \cdot \omega \cdot t}=-\rho_{0} \cdot \frac{\partial\left(\phi \cdot e^{-i \cdot \omega \cdot t}\right)}{\partial t} \\
p \cdot e^{-i \cdot \omega \cdot t}=-\rho_{0} \cdot \phi \cdot e^{-i \cdot \omega \cdot t} \cdot(-i \cdot \omega) \\
p=i \cdot \omega \cdot \rho_{0} \cdot \phi
\end{gathered}
$$

Derivando-se a expressão (5.1) em relação à direção normal, no sentido positivo do fluido, tem-se:

$$
\begin{aligned}
& \frac{\partial p}{\partial \eta}=i \cdot \omega \cdot \rho_{0} \cdot \frac{\partial \phi}{\partial \eta} \\
& \frac{\partial p}{\partial \eta}=i \cdot \omega \cdot \rho_{0} \cdot \nabla \phi \\
& \frac{\partial p}{\partial \eta}=i \cdot \omega \cdot \rho_{0} \cdot \vec{v}
\end{aligned}
$$

No domínio da freqüência, admite-se $\vec{v}=\omega \cdot \vec{u}$. Considerando-se $u$ positivo no sentido de $\eta$ e negativo no sentido contrário, pode-se escrever $\vec{v}=\omega \cdot u$.

$$
\begin{gathered}
\frac{\partial p}{\partial \eta}=i \cdot \omega \cdot \rho_{0} \cdot(\omega \cdot u) \\
\frac{\partial p}{\partial \eta}=i \cdot \omega^{2} \cdot \rho_{0} \cdot u
\end{gathered}
$$

Assim, a equação integral de contorno do fluido (2.20) pode ser rescrita como:

$$
c_{(S)} \cdot p_{(S)}+\int_{\Gamma} \frac{\partial p^{*}}{\partial \eta} \cdot p d_{\Gamma}=i \cdot \omega^{2} \cdot \rho_{0} \cdot \int_{\Gamma} p^{*} \cdot u d_{\Gamma}-\int_{\Omega} D \cdot p^{*} d_{\Omega}
$$

Na forma matricial, tem-se:

$$
\begin{gathered}
{\left[H_{F}\right] \cdot\left\{p_{F}\right\}=i \cdot \omega^{2} \cdot \rho_{0} \cdot\left[\bar{G}_{F}\right] \cdot\left\{u_{F}\right\}-\left\{D_{F}\right\}} \\
{\left[H_{F}\right] \cdot\left\{p_{F}\right\}=\left[G_{F}\right] \cdot\left\{u_{F}\right\}-\left\{D_{F}\right\} \quad \text { (equação matricial do fluido) }}
\end{gathered}
$$

O sistema de coordenadas em que é feito o acoplamento é o do fluido $(\Gamma, \eta)$. Devese fazer uma transformação de coordenadas da estrutura $(X, Y)$ para o sistema de coordenadas do fluido. 


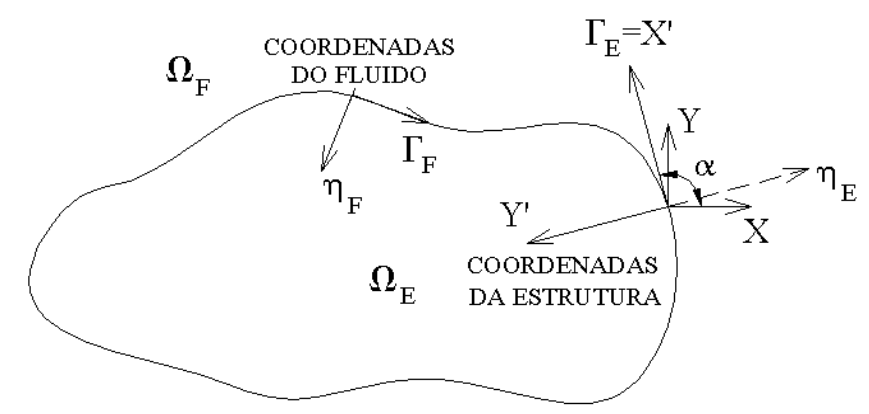

Figura 5.1 - Sistemas de coordenadas do fluido e da estrutura

A transformação utilizada rotaciona o sistema $X Y$, segundo um ângulo $\alpha$, de modo que $X$ tenha o mesmo sentido de $\Gamma$ da estrutura (anti-horário), com sentido contrário ao $\Gamma$ do fluido.

Onde $\alpha$ é o ânguld ${ }^{3}$ do elemento de contorno da estrutura onde a transformação está sendo feita; portanto, muda de acordo com as colunas do sistema matricial (4.22) da estrutura.

As transformações são processadas nos vetores das variáveis de contorno $\{u\}$ e $\{p\}$, orientadas pelo sistema $X Y$, para os vetores de variáveis $\left\{u_{E}\right\}$ e $\left\{p_{E}\right\}$, também de contorno, orientadas pelo sistema $\Gamma \eta$.

$$
\begin{gathered}
{[H] \cdot\{u\}=[G] \cdot\{p\}} \\
{[H] \cdot[\beta] \cdot\left\{u_{E}\right\}=[G] \cdot[\beta] \cdot\left\{p_{E}\right\}} \\
{\left[H_{E}\right] \cdot\left\{u_{E}\right\}=\left[G_{E}\right] \cdot\left\{p_{E}\right\} \quad \text { (equação matricial da estrutura) }}
\end{gathered}
$$

Onde:

$$
[\beta]=\left[\begin{array}{cc}
\cos \alpha & -\operatorname{sen} \alpha \\
\operatorname{sen} \alpha & \cos \alpha
\end{array}\right]
$$

Exemplificando, para o vetor $\{u\}$, tem-se:

$$
\begin{aligned}
& \{u\}=[\beta] \cdot\left\{u_{E}\right\} \\
& \left\{\begin{array}{c}
\left\{\begin{array}{l}
u_{1} \\
u_{2}
\end{array}\right\}_{1} \\
\left\{\begin{array}{c}
u_{1} \\
u_{2}
\end{array}\right\}_{2} \\
\vdots
\end{array}\right\}=\left[\begin{array}{ccccc}
\cos \alpha_{1} & -\operatorname{sen} \alpha_{1} & 0 & 0 & \ldots \\
\operatorname{sen} \alpha_{1} & \cos \alpha_{1} & 0 & 0 & \ldots \\
0 & 0 & \cos \alpha_{2} & -\operatorname{sen} \alpha_{2} & \ldots \\
0 & 0 & \operatorname{sen} \alpha_{2} & \cos \alpha_{2} & \ldots \\
\vdots & \vdots & \vdots & \vdots & \ddots
\end{array}\right] \cdot\left\{\begin{array}{c}
u_{\Gamma} \\
\left.u_{\eta}\right\}_{1} \\
\vdots \\
u_{\Gamma} \\
\left.u_{\eta}\right\}_{2} \\
\vdots
\end{array}\right\}
\end{aligned}
$$

\footnotetext{
${ }^{3}$ Medido no sentido anti-horário.
} 


\subsection{Sistema acoplado de equações}

A equação integral de contorno escrita na forma matricial (5.5) pode ser decomposta em uma parcela acoplada (ou de interface), representada pelo índice superior $i$, e em outra parcela não acoplada (ou externa), representada pelo índice superior $e$. São acopladas apenas as variáveis com orientação segundo a direção normal $(\eta)$ ao contorno.

$$
\left[\begin{array}{ll}
G_{F}^{e} & G_{F}^{i}
\end{array}\right] \cdot\left\{\begin{array}{l}
u_{F}^{e} \\
u_{F}^{i}
\end{array}\right\}=\left[\begin{array}{ll}
H_{F}^{e} & H_{F}^{i}
\end{array}\right] \cdot\left\{\begin{array}{l}
p_{F}^{e} \\
p_{F}^{i}
\end{array}\right\}+\left\{D_{F}\right\}
$$

Para a estrutura, utiliza-se a representação decomposta da equação matricial (5.6).

$$
\left[\begin{array}{ll}
H_{E}^{e} & H_{E}^{i}
\end{array}\right] \cdot\left\{\begin{array}{l}
u_{E}^{e} \\
u_{E}^{i}
\end{array}\right\}=\left[\begin{array}{ll}
G_{E}^{e} & G_{E}^{i}
\end{array}\right] \cdot\left\{\begin{array}{c}
p_{E}^{e} \\
p_{E}^{i}
\end{array}\right\}
$$

São admitidas as seguintes condições de contorno para as variáveis de interface acopladas:

(a) Deslocamentos iguais: $u_{F}^{i}=u_{E}^{i}=u_{i}$

(b) Forças de superfície em equilíbrio: $p_{F}^{i}+p_{E}^{i}=0 \Rightarrow p_{F}^{i}=-p_{E}^{i}=p_{i}$

Combinando as equações (5.8) e (5.9) e levando-se em consideração as condições de equilíbrio e compatibilidade na interface, obtém-se o sistema acoplado de equações.

$$
\left[\begin{array}{cccc}
H_{E}^{e} & H_{E}^{i} & G_{E}^{i} & 0 \\
0 & G_{F}^{i} & -H_{F}^{i} & G_{F}^{e}
\end{array}\right] \cdot\left\{\begin{array}{c}
u_{E}^{e} \\
u_{i} \\
p_{i} \\
u_{F}^{e}
\end{array}\right\}=\left[\begin{array}{cccc}
G_{E}^{e} & G_{E}^{i} & 0 & 0 \\
0 & 0 & H_{F}^{i} & H_{F}^{e}
\end{array}\right] \cdot\left\{\begin{array}{c}
p_{E}^{e} \\
\bar{p}_{E}^{i} \\
\bar{p}_{F}^{i} \\
p_{F}^{e}
\end{array}\right\}+\left\{\begin{array}{c}
0 \\
D_{F}
\end{array}\right\}
$$

Onde:

$\bar{p}_{E}^{i}$ e $\bar{p}_{F}^{i}$ são valores prescritos de forças de superfície aplicadas nas interfaces da estrutura e do fluido respectivamente.

Como exemplo literal de acoplamento é analisado o caso de um elemento de contorno na interface entre o fluido e a estrutura. Os sistemas de coordenadas já estão orientados segundo o fluido.

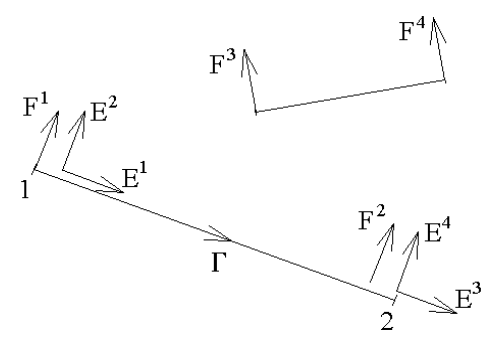

Figura 5.2 - Exemplo de acoplamento fluido-estrutura em um elemento de contorno na interface 
Para o fluido tem-se:

$$
\left[\begin{array}{llll}
G_{F}^{11} & G_{F}^{12} & G_{F}^{13} & G_{F}^{14} \\
G_{F}^{21} & G_{F}^{22} & G_{F}^{23} & G_{F}^{24} \\
G_{F}^{31} & G_{F}^{32} & G_{F}^{33} & G_{F}^{34} \\
G_{F}^{41} & G_{F}^{42} & G_{F}^{43} & G_{F}^{44}
\end{array}\right] \cdot\left\{\begin{array}{l}
u_{F}^{1} \\
u_{F}^{2} \\
u_{F}^{3} \\
u_{F}^{4}
\end{array}\right\}=\left[\begin{array}{cccc}
H_{F}^{11} & H_{F}^{12} & H_{F}^{13} & H_{F}^{14} \\
H_{F}^{21} & H_{F}^{22} & H_{F}^{23} & H_{F}^{24} \\
H_{F}^{31} & H_{F}^{32} & H_{F}^{33} & H_{F}^{34} \\
H_{F}^{41} & H_{F}^{42} & H_{F}^{43} & H_{F}^{44}
\end{array}\right] \cdot\left\{\begin{array}{c}
p_{F}^{1} \\
p_{F}^{2} \\
p_{F}^{3} \\
p_{F}^{4}
\end{array}\right\}+\left\{\begin{array}{c}
D_{F}^{1} \\
D_{F}^{2} \\
D_{F}^{3} \\
D_{F}^{4}
\end{array}\right\}
$$

Para a estrutura tem-se:

$$
\left[\begin{array}{llll}
H_{E}^{11} & H_{E}^{12} & H_{E}^{13} & H_{E}^{14} \\
H_{E}^{21} & H_{E}^{22} & H_{E}^{23} & H_{E}^{24} \\
H_{E}^{31} & H_{E}^{32} & H_{E}^{33} & H_{E}^{34} \\
H_{E}^{41} & H_{E}^{42} & H_{E}^{43} & H_{E}^{44}
\end{array}\right] \cdot\left\{\begin{array}{l}
u_{E}^{1} \\
u_{E}^{2} \\
u_{E}^{3} \\
u_{E}^{4}
\end{array}\right\}=\left[\begin{array}{llll}
G_{E}^{11} & G_{E}^{12} & G_{E}^{13} & G_{E}^{14} \\
G_{E}^{21} & G_{E}^{22} & G_{E}^{23} & G_{E}^{24} \\
G_{E}^{31} & G_{E}^{32} & G_{E}^{33} & G_{E}^{34} \\
G_{E}^{41} & G_{E}^{42} & G_{E}^{43} & G_{E}^{44}
\end{array}\right] \cdot\left\{\begin{array}{c}
p_{E}^{1} \\
p_{E}^{2} \\
p_{E}^{3} \\
p_{E}^{4}
\end{array}\right\}
$$

No acoplamento são admitidas as seguintes condições de contorno:

$$
\begin{gathered}
u_{F}^{1}=u_{E}^{2}=u_{i}^{1} \\
u_{F}^{2}=u_{E}^{4}=u_{i}^{2} \\
p_{F}^{1}=-p_{E}^{2}=p_{i}^{1} \\
p_{F}^{2}=-p_{E}^{4}=p_{i}^{2}
\end{gathered}
$$

Com base na equação (5.10) monta-se o sistema acoplado de equações.

$$
\begin{aligned}
& {\left[\begin{array}{cccccccc}
H_{E}^{11} & H_{E}^{13} & H_{E}^{12} & H_{E}^{14} & G_{E}^{12} & G_{E}^{14} & 0 & 0 \\
H_{E}^{21} & H_{E}^{23} & H_{E}^{22} & H_{E}^{24} & G_{E}^{22} & G_{E}^{24} & 0 & 0 \\
H_{E}^{31} & H_{E}^{33} & H_{E}^{32} & H_{E}^{34} & G_{E}^{32} & G_{E}^{34} & 0 & 0 \\
H_{E}^{41} & H_{E}^{43} & H_{E}^{42} & H_{E}^{44} & G_{E}^{42} & G_{E}^{44} & 0 & 0 \\
0 & 0 & G_{F}^{11} & G_{F}^{12} & -H_{F}^{11} & -H_{F}^{12} & G_{F}^{13} & G_{F}^{14} \\
0 & 0 & G_{F}^{21} & G_{F}^{22} & -H_{F}^{21} & -H_{F}^{22} & G_{F}^{23} & G_{F}^{24} \\
0 & 0 & G_{F}^{31} & G_{F}^{32} & 0 & 0 & G_{F}^{33} & G_{F}^{34} \\
0 & 0 & G_{F}^{41} & G_{F}^{42} & 0 & 0 & G_{F}^{43} & G_{F}^{44}
\end{array}\right] \cdot\left\{\begin{array}{c}
u_{E}^{1} \\
u_{E}^{3} \\
u_{i}^{1} \\
u_{i}^{2} \\
p_{i}^{1} \\
p_{i}^{2} \\
u_{F}^{3} \\
u_{F}^{4}
\end{array}\right\}=} \\
& {\left[\begin{array}{ccccccccc}
G_{E}^{11} & G_{E}^{13} & G_{E}^{12} & G_{E}^{14} & 0 & 0 & 0 & 0 \\
G_{E}^{21} & G_{E}^{23} & G_{E}^{22} & G_{E}^{24} & 0 & 0 & 0 & 0 \\
G_{E}^{31} & G_{E}^{33} & G_{E}^{32} & G_{E}^{34} & 0 & 0 & 0 & 0 \\
G_{E}^{41} & G_{E}^{43} & G_{E}^{42} & G_{E}^{44} & 0 & 0 & 0 & 0 \\
0 & 0 & 0 & 0 & H_{F}^{11} & H_{F}^{12} & H_{F}^{13} & H_{F}^{14} \\
0 & 0 & 0 & 0 & H_{F}^{21} & H_{F}^{22} & H_{F}^{23} & H_{F}^{24} \\
0 & 0 & 0 & 0 & H_{F}^{31} & H_{F}^{32} & H_{F}^{33} & H_{F}^{34} \\
0 & 0 & 0 & 0 & H_{F}^{41} & H_{F}^{42} & H_{F}^{43} & H_{F}^{44}
\end{array}\right] \cdot\left\{\begin{array}{l}
p_{E}^{1} \\
p_{E}^{3} \\
\bar{p}_{E}^{2} \\
\bar{p}_{E}^{4} \\
\bar{p}_{F}^{1} \\
\bar{p}_{F}^{2} \\
p_{F}^{3} \\
p_{F}^{4}
\end{array}\right\}+\left\{\begin{array}{c}
0 \\
0 \\
0 \\
0 \\
D_{F}^{1} \\
D_{F}^{2} \\
D_{F}^{3} \\
D_{F}^{4}
\end{array}\right\}}
\end{aligned}
$$


Separando-se as variáveis prescritas das incógnitas no sistema (5.14) em lados opostos da igualdade, obtém-se um sistema final de equações do tipo $[A] \cdot\{X\}=\{F\}$.

\subsection{Exemplo numérico}

O exemplo de acoplamento fluido-estrutura é constituído de uma chapa elastodinâmica, em EPT, acoplada com o ar $\left(20^{\circ} \mathrm{C}\right)$. O carregamento é aplicado sobre a interface, na estrutura, a uma freqüência de $20 \mathrm{~Hz}$. O contorno é discretizado em 140 elementos lineares, de tamanhos $L=0.1 \mathrm{~m}$, com distribuições lineares de variáveis (DL_140).

Para o fluido é utilizada a técnica de integração TC3, com um ponto de CHIEF no CG da chapa. São dados do fluido: $\rho_{0}=1.21 \mathrm{Kg} / \mathrm{m}^{3}, c=343 \mathrm{~m} / \mathrm{s} ; \bar{p}_{F}^{i}=0 \mathrm{~N} / \mathrm{m}$.

Para a estrutura é utilizada a técnica de integração TC1, com distância de afastamento dos pontos de colocação aos elementos de contorno igual a $L / 50$, no sentido chapa-fluido. São dados da estrutura: $E=19.5 \cdot 10^{10} \mathrm{~Pa}, v=0.28, \rho=7700 \mathrm{Kg} / \mathrm{m}^{3}$, $\bar{p}_{E \text { min } \text {.dir.X }}^{i}=-100000 \mathrm{~N} / \mathrm{m} ; \bar{p}_{E \text { máx.dir.X }}^{i}=100000 \mathrm{~N} / \mathrm{m}$.

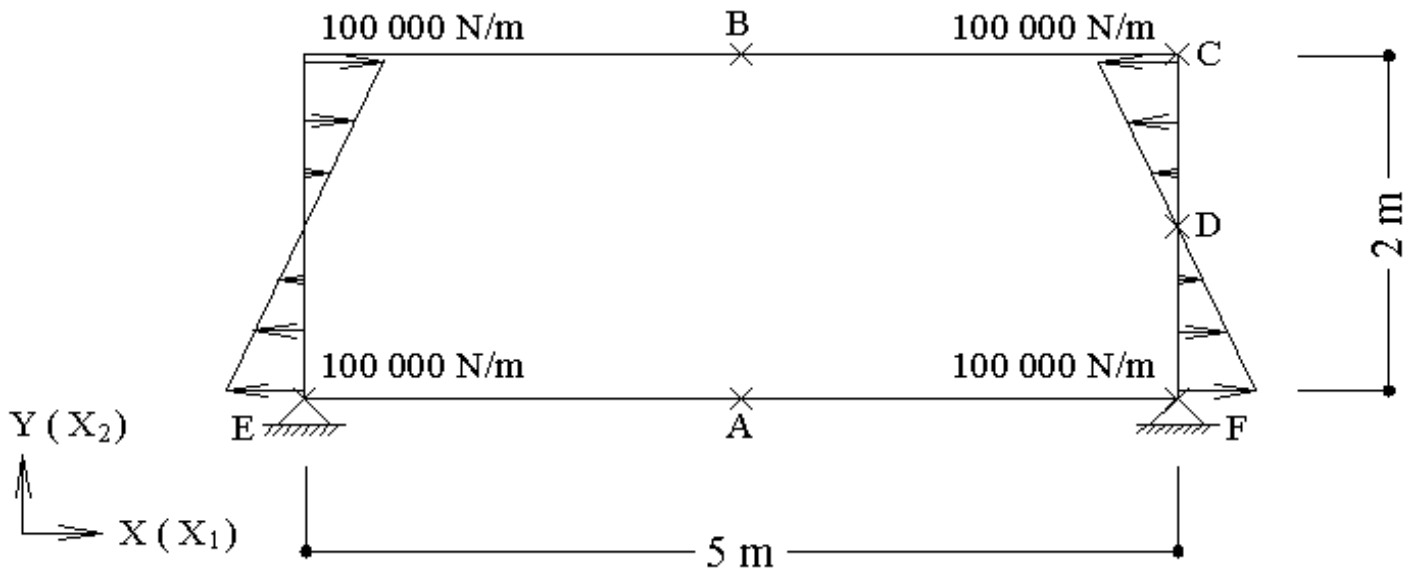

Figura 5.3 - Geometria, condições de contorno e pontos críticos no contorno do problema

As respostas obtidas pelo acoplamento MEC-MEC são comparadas com as do programa ANSYS (MEF-MEF). Foram utilizados, no ANSYS, elementos finitos PLANE42 (1000) na modelagem da chapa e os elementos finitos FLUID29 (7610) e FLUID129 (200) na modelagem do fluido. As distribuições dos elementos finitos nas linhas que definem a geometria estão representadas na figura seguinte. 


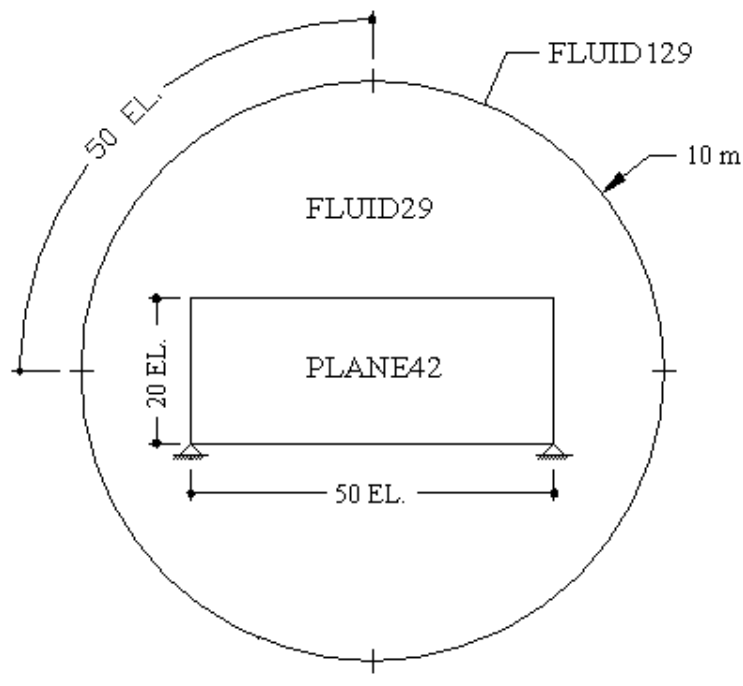

Figura 5.4 - Contornos da malha de elementos finitos utilizada no programa ANSYS

O elemento finito FLUID129, utilizado apenas em conjunto com FLUID29, simula as condições de contorno no limite infinito do fluido.

Tabela 5.1 - Deslocamentos (UX, UY) em pontos críticos no contorno

\begin{tabular}{||c|c|c|c|c|c|c||}
\hline \multirow{2}{*}{ PONTO } & \multicolumn{2}{|c|}{ MEF (ANSYS) } & \multicolumn{2}{c|}{ MEC - TC1 (L/50) } & \multirow{2}{*}{ ERRO RELATIVO (\%) } \\
\cline { 2 - 7 } & UX [m] & UY [m] & UX [m] & UY [m] & X & Y \\
\hline A & $3.4261 \mathrm{E}-18$ & $-6.2398 \mathrm{E}-07$ & $9.6575 \mathrm{E}-11$ & $-5.9940 \mathrm{E}-07$ & - & $4 \%$ \\
\hline B & $-4.7296 \mathrm{E}-17$ & $-5.9268 \mathrm{E}-07$ & $-8.9553 \mathrm{E}-11$ & $-5.6743 \mathrm{E}-07$ & - & $4 \%$ \\
\hline C & $-1.0886 \mathrm{E}-06$ & $4.9938 \mathrm{E}-07$ & $-1.0896 \mathrm{E}-06$ & $5.2297 \mathrm{E}-07$ & $0 \%$ & $5 \%$ \\
\hline D & $-7.9281 \mathrm{E}-08$ & $3.9043 \mathrm{E}-07$ & $-8.2089 \mathrm{E}-08$ & $4.1516 \mathrm{E}-07$ & $3 \%$ & $6 \%$ \\
\hline
\end{tabular}

Tabela 5.2 - Reações de apoio

\begin{tabular}{||c|c|c|c|c||}
\hline \multirow{2}{*}{ PONTO } & \multicolumn{2}{|c|}{ MEF (ANSYS) } & \multicolumn{2}{c||}{ MEC - TC1 (L/50) } \\
\cline { 2 - 5 } & FX [N] & FY [N] & FX [N] & FY [N] \\
\hline E & 23552 & 178 & 24164 & 117 \\
\hline F & -23552 & 178 & -24164 & 117 \\
\hline
\end{tabular}

Para o cálculo dos deslocamentos na chapa, obtidos pelo MEC, foram utilizados 133 pontos internos. Os campos de deslocamento em X e Y estão ilustrados nas figuras a seguir. 


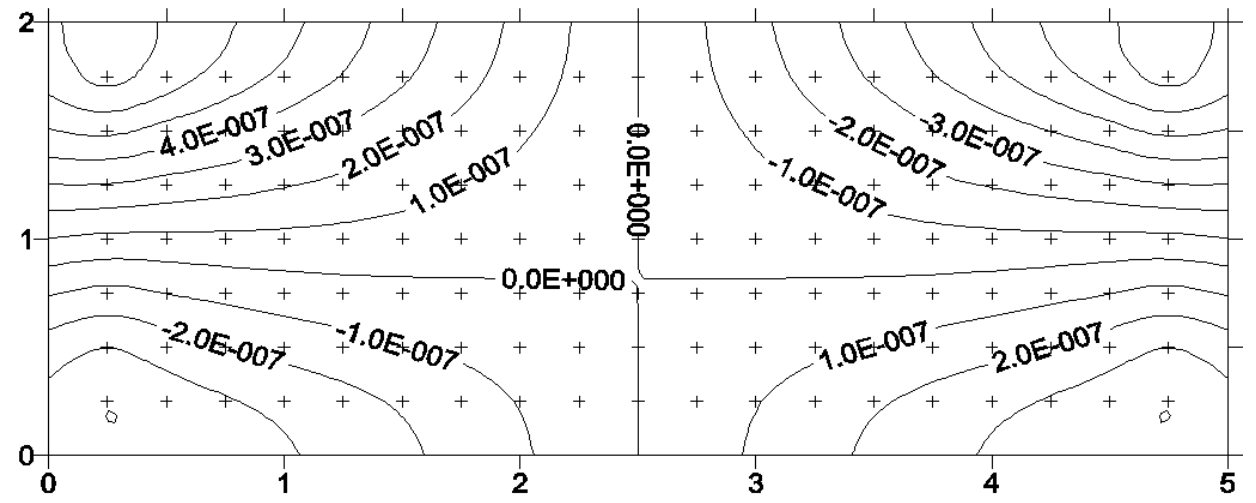

Figura 5.5 - Campo de deslocamentos da chapa na direção X [m]

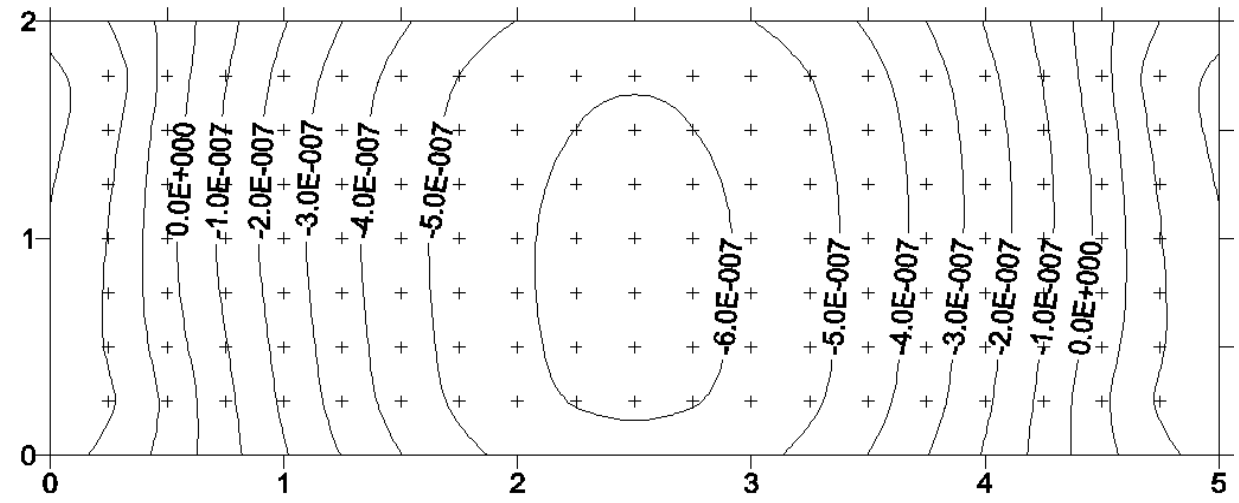

Figura 5.6 - Campo de deslocamentos da chapa na direção Y [m]

São apresentados também os deslocamentos em pontos internos da chapa obtidos através do programa ANSYS.

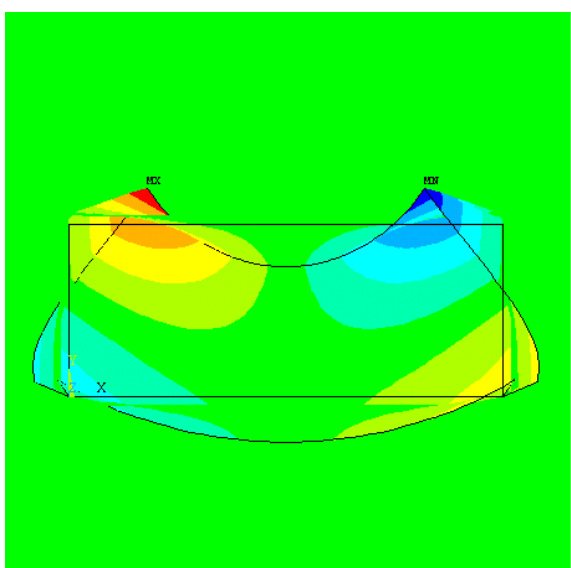

Figura 5.7 - Deslocamentos da chapa na direção $\mathrm{X}$ [m], obtidos pelo programa ANSYS

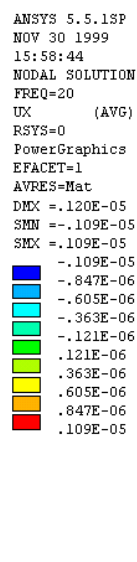

Figura 5.8 - Deslocamentos da chapa na direção $\mathrm{Y}$ [m], obtidos pelo programa ANSYS 


\section{CONCLUSÕES}

Foram estudados problemas bidimensionais de radiação e difusãd acústica, além de problemas de acoplamento fluido-estrutura, formulados através do Método dos Elementos de Contorno (MEC).

Nos problemas de radiação e difusão acústica, com o meio de propagação das ondas acústicas ${ }^{2}$ modelado via MEC, foram estudadas três técnicas de integração dos núcleos integrais: ponto de colocação fora do domínio com utilização de sub-elementação (TC1), ponto de colocação no contorno (TC2) e ponto de colocação no contorno com correção do CHIEF (TC3). Com base nos exemplos numéricos apresentados no capítulo 3, observou-se que a técnica $\mathrm{TC} 1$ foi a mais versátil, pois forneceu bons resultados tanto para domínios finitos como para infinitos (com ou sem termos de domínio). A técnica TC2 apresentou bons resultados apenas nos casos de domínios finitos e infinitos com termos de domínio (núcleos de pressão acústica do exemplo 7). A estratégia de integração de TC3 é a mais utilizada na bibliografia sobre o assunto e forneceu bons resultados para os problemas de domínios infinitos analisados, resolveu o problema da não unicidade de resposta pertinente à formulação integral de contorno; para domínios finitos e infinitos com termos de domínio forneceu praticamente os mesmos resultados de TC2. Comparando-se as respostas das três técnicas empregadas neste trabalho com as soluções analíticas e respostas via Método dos Elementos Finitos (MEF) dos exemplos numéricos do capítulo 3, recomenda-se que seja utilizada TC1 ou TC2 para problemas envolvendo domínios finitos e TC3 para domínios infinitos. De uma maneira geral as respostas obtidas via MEC em problemas de radiação acústica foram de boa qualidade. Para o problema de difusão acústica (exemplos 7 e 8) o $\mathrm{MEC}$, com o uso da técnica TC1, forneceu respostas coerentes com a teoria física da Acústica, se mostrando uma ferramenta útil para este tipo de análise.

Quanto ao uso do MEC em problemas de elastodinâmica, baseando-se no exemplo numérico do capítulo 4 com respostas analisadas em várias freqüências de carregamento,

\footnotetext{
${ }^{1}$ Modelada através de núcleos de pressão (fontes acústicas) gerando as ondas incidentes na superfície analisada.

${ }^{2}$ Fluido compressível.
} 
pode-se dizer que os resultados foram bons, quando comparados com os obtidos através do programa ANSYS (MEF). A técnica de integração utilizada neste caso foi a do ponto de colocação fora do domínio com utilização de sub-elementação (análoga a TC1 aplicada no caso do fluido).

Para o caso de acoplamento entre o fluido e a estrutura, a formulação totalmente baseada no MEC mostrou-se eficiente no caso de uma chapa acoplada com o ar (exemplo numérico do capítulo 5), os resultados foram próximos dos obtidos pelo MEF (ANSYS). Uma outra possibilidade de acoplamento fluido-estrutura, mais comum na bibliografia, baseia-se na utilização do MEC na modelagem numérica do fluido e do MEF na modelagem da estrutura. A principal vantagem do acoplamento MEC-MEC, quando comparado com MEC-MEF, é a montagem direta do sistema acoplado de equações. Não há necessidade de transformar uma parte do sistema de MEC para MEF, ou vice-versa. Outro aspecto importante é a possibilidade de utilização de nós descontínuos, que são úteis em formulações do MEC, nos contornos do fluido e da estrutura, fato que simplifica o acoplamento nodal.

O MEC aplicado em problemas de acústica e acoplamento fluido-estrutura apresentou grande potencial, especialmente em problemas envolvendo domínios infinitos sendo atualmente o método numérico mais recomendado para este tipo de análise.

Em termos de trabalhos futuros é recomendável que sejam implementadas computacionalmente uma rotina que considere as soluções fundamentais ${ }^{4}$ para problemas de domínio semi-infinito (solução fundamental em espelho) e uma outra rotina que considere o ponto de colocação no contorno para o programa elastodinâmico no domínio da freqüência. Uma outra possibilidade imediata é o estudo de uma estrutura acoplada através de duas interfaces de fluido, combinando-se ainda o problema da difusão acústica (onde podem existir núcleos de pressão em um ou dois meios fluidos). É claro que existem inúmeras outras idéias para trabalhos futuros, como o estudo de problemas semelhantes aos apresentados nesta dissertação em três dimensões. Cabe aos interessados pelo assunto encontrarem, com muito trabalho e criatividade, novas formas de aplicação e extensão desta dissertação.

\footnotetext{
${ }^{3}$ Caso de fluido não confinado.

${ }^{4}$ Dos programas de modelagem do meio fluido e do meio elástico (estrutura).
} 


\section{ANEXO A}

\section{Funções de Bessel utilizadas}

\section{A.1 Funções de Hankel de $1^{\text {a }}$ classe}

A solução fundamental, apresentada no item 2.2 .2 , e sua derivada em relação à reta normal são constituídas de funções de Hankel de $1^{\text {a }}$ classe de ordem 0 e 1 , respectivamente.

$$
\begin{aligned}
& H_{0}^{(1)}=J_{0}(x)+i \cdot Y_{0}(x) \\
& H_{1}^{(1)}=J_{1}(x)+i \cdot Y_{1}(x)
\end{aligned}
$$

$J_{n}(x)$ é uma função de Bessel do primeiro tipo, representada por:

$$
J_{n}(x)=\sum_{k=0}^{\infty} \frac{(-1)^{k} \cdot\left(\frac{x}{2}\right)^{2 \cdot k+n}}{k ! \cdot \Gamma(k+n+1)}
$$

Onde:

$n=0,1,2, \ldots$

Função Gama: $\Gamma(m+1)=m$ !

$Y_{n}(x)$ é uma função de Bessel do segundo tipo representada por:

$$
\begin{aligned}
Y_{n}(x) & =\frac{2}{\pi} \cdot\left[\ln \left(\frac{x}{2}\right)+\gamma\right] \cdot J_{n}(x)-\frac{1}{\pi} \cdot \sum_{k=0}^{n-1}(n-k-1) ! \cdot\left(\frac{x}{2}\right)^{2 \cdot k-n} \\
& -\frac{1}{\pi} \cdot \sum_{k=0}^{\infty}(-1)^{k} \cdot[\Phi(k)+\Phi(n+k)] \cdot \frac{\left(\frac{x}{2}\right)^{2 \cdot k+n}}{k ! \cdot(n+k) !}
\end{aligned}
$$

Onde:

$n=0,1,2, \ldots$

Constante de Euler: $\gamma \cong 0.5772156649015329$

$\Phi(p)=\sum_{m=1}^{p}\left(\frac{1}{m}\right), \Phi(0)=0$ 




Figura A.1 - Funções de Bessel do primeiro tipo de ordem 0 e 1

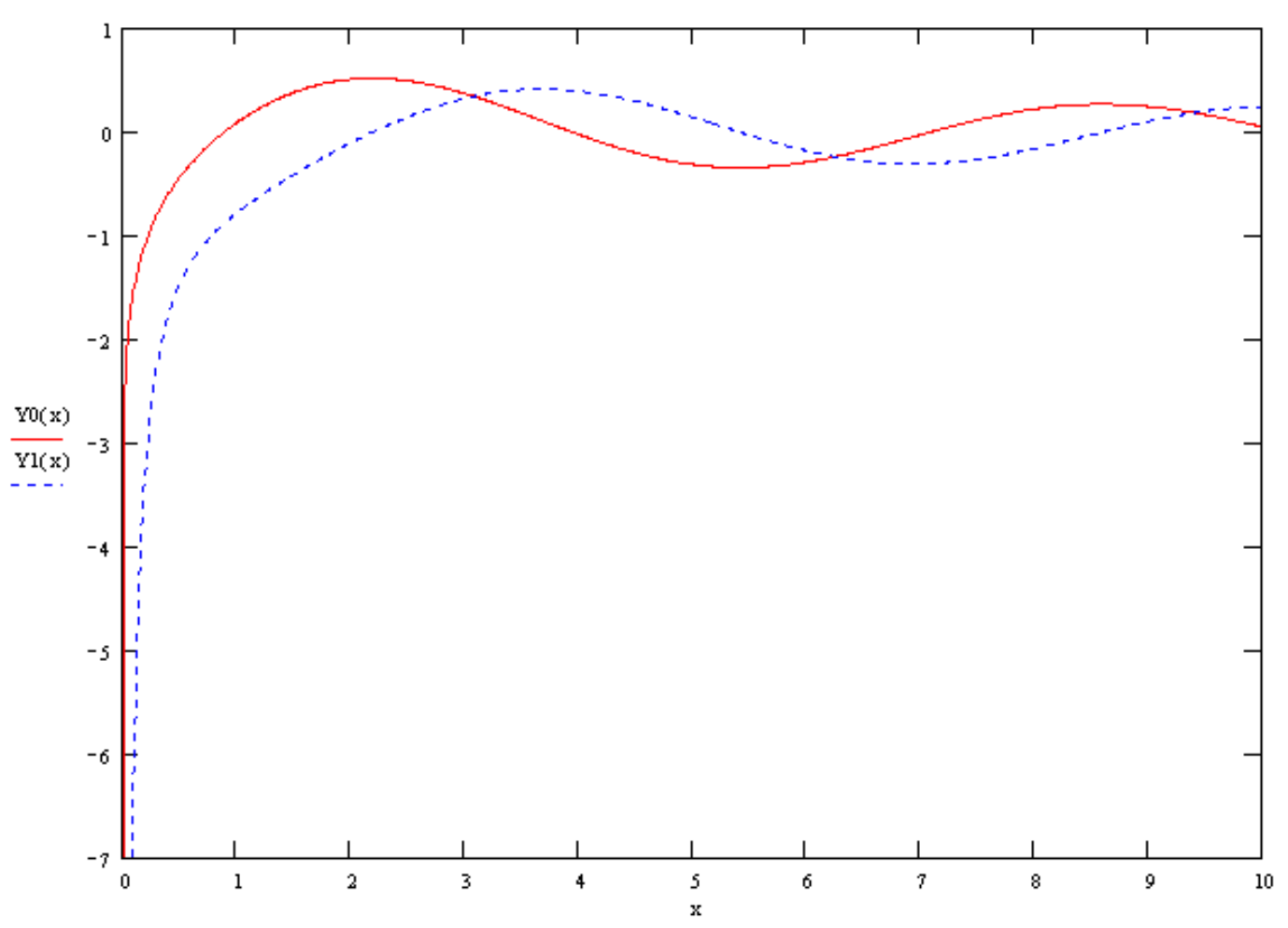

Figura A.2 - Funções de Bessel do segundo tipo de ordem 0 e 1 


\section{A.2 Funções modificadas de Bessel}

Na solução fundamental do problema elastodinâmico harmônico e na equação de $p_{l m}^{*}$ apresentadas no item 4.2 são utilizadas funções modificadas de Bessel de $2^{\mathrm{a}}$ classe e ordens $0,1 \mathrm{e} 2$.

$K_{n}(x)$ é uma função modificada de Bessel de $2^{\text {a }}$ classe e ordem $n$, expressa por:

$$
\begin{aligned}
K_{n}(x) & =(-1)^{n+1} \cdot\left[\ln \left(\frac{x}{2}\right)+\gamma\right] \cdot I_{n}(x)+\frac{1}{2} \cdot \sum_{k=0}^{n-1}(-1)^{k} \cdot(n-k-1) ! \cdot\left(\frac{x}{2}\right)^{2 \cdot k-n} \\
& +\frac{(-1)^{n}}{2} \cdot \sum_{k=0}^{\infty} \frac{\left(\frac{x}{2}\right)^{2 \cdot k+n}}{k ! \cdot(n+k) !} \cdot[\Phi(k)+\Phi(n+k)]
\end{aligned}
$$

Onde:

$n=0,1,2, \ldots$

$I_{n}(x)$ é uma função modificada de Bessel de $1^{\text {a }}$ classe e ordem $n$, expressa por:

$I_{n}(x)=i^{-n} \cdot J_{n}(i \cdot x)$.

Os programas desenvolvidos nesta dissertação utilizam para o cálculo das funções de Bessel, um conjunto de rotinas e funções prontas, internas do FORTRAN PowerStation 4.0 (1994-95), chamado de biblioteca interna de subrotinas matemáticas (IMSL) da Microsoft Corporation ${ }^{\circledR}$. 


\section{ANEXO B}

Fluxograma do acoplamento fluido-estrutura
















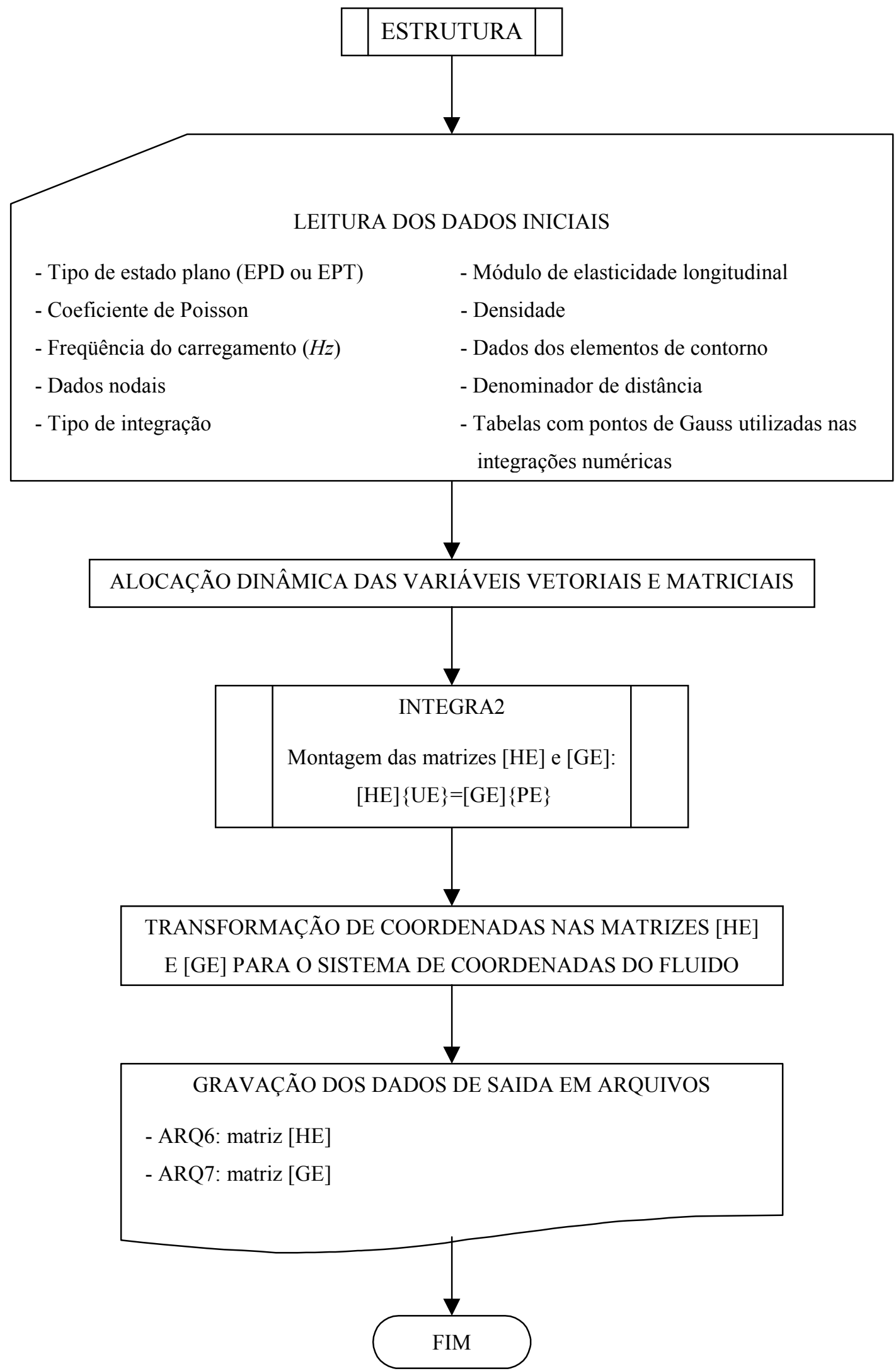




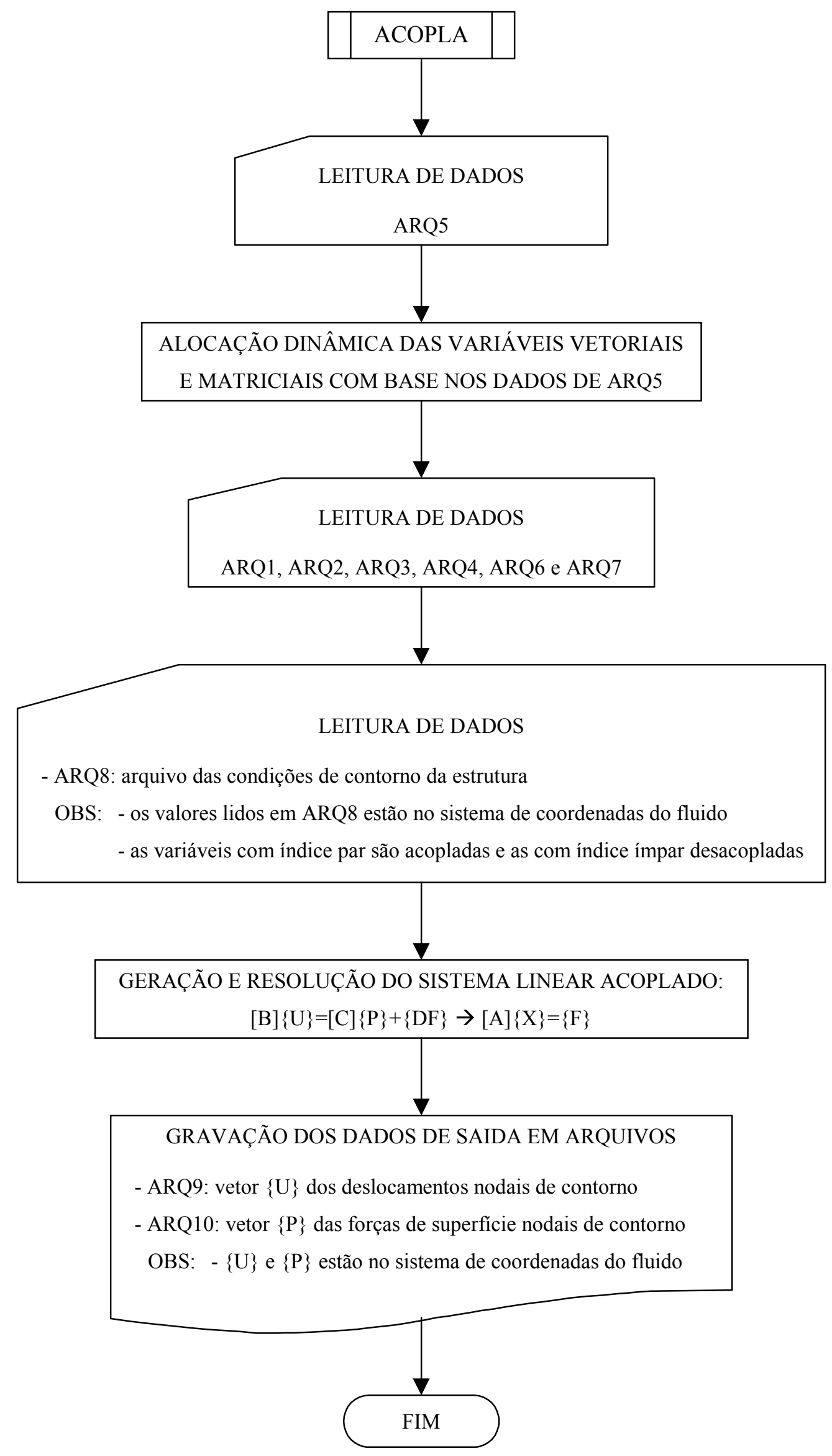









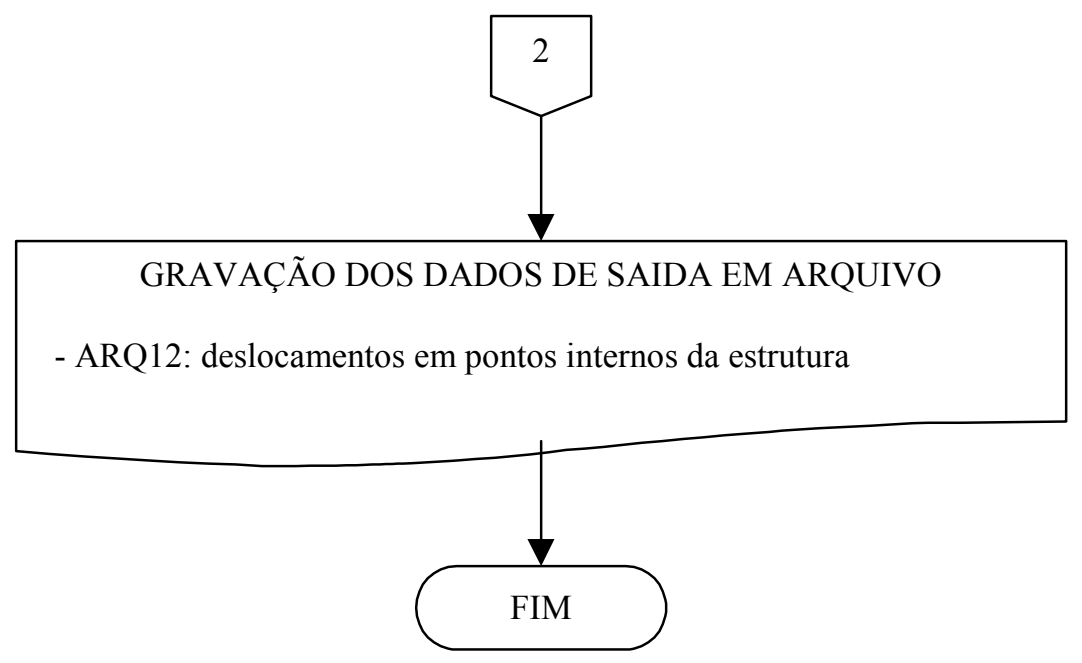

Para o conjunto de programas do acoplamento fluido-estrutura, esquematizado acima, utilizaram-se variáveis de contorno com distribuições lineares. Também foram feitos programas de propagação de ondas acústicas para o fluido, com distribuições constantes e lineares para as variáveis de contorno, e um programa elastodinâmico para a estrutura, com distribuições lineares para as variáveis de contorno. 


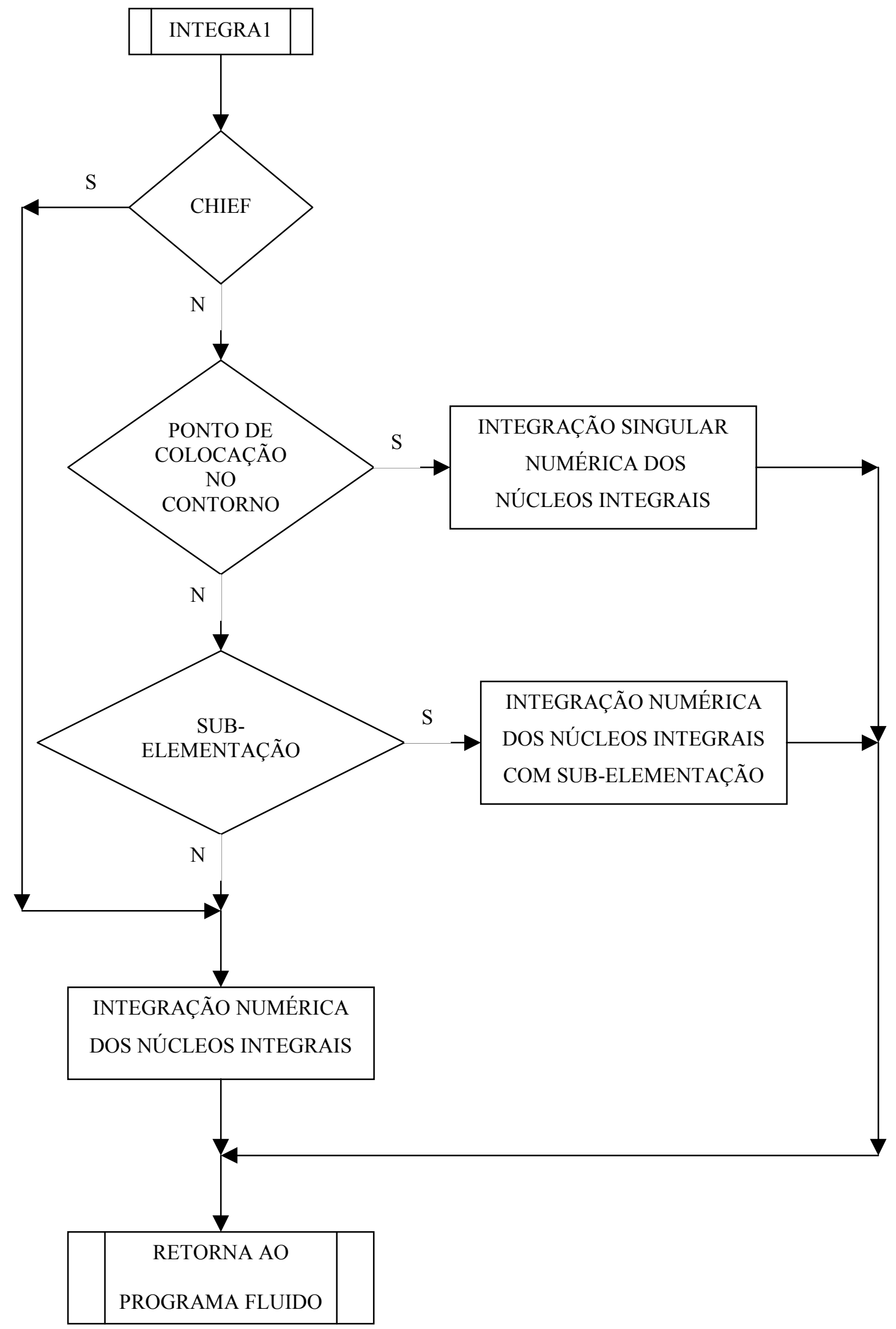




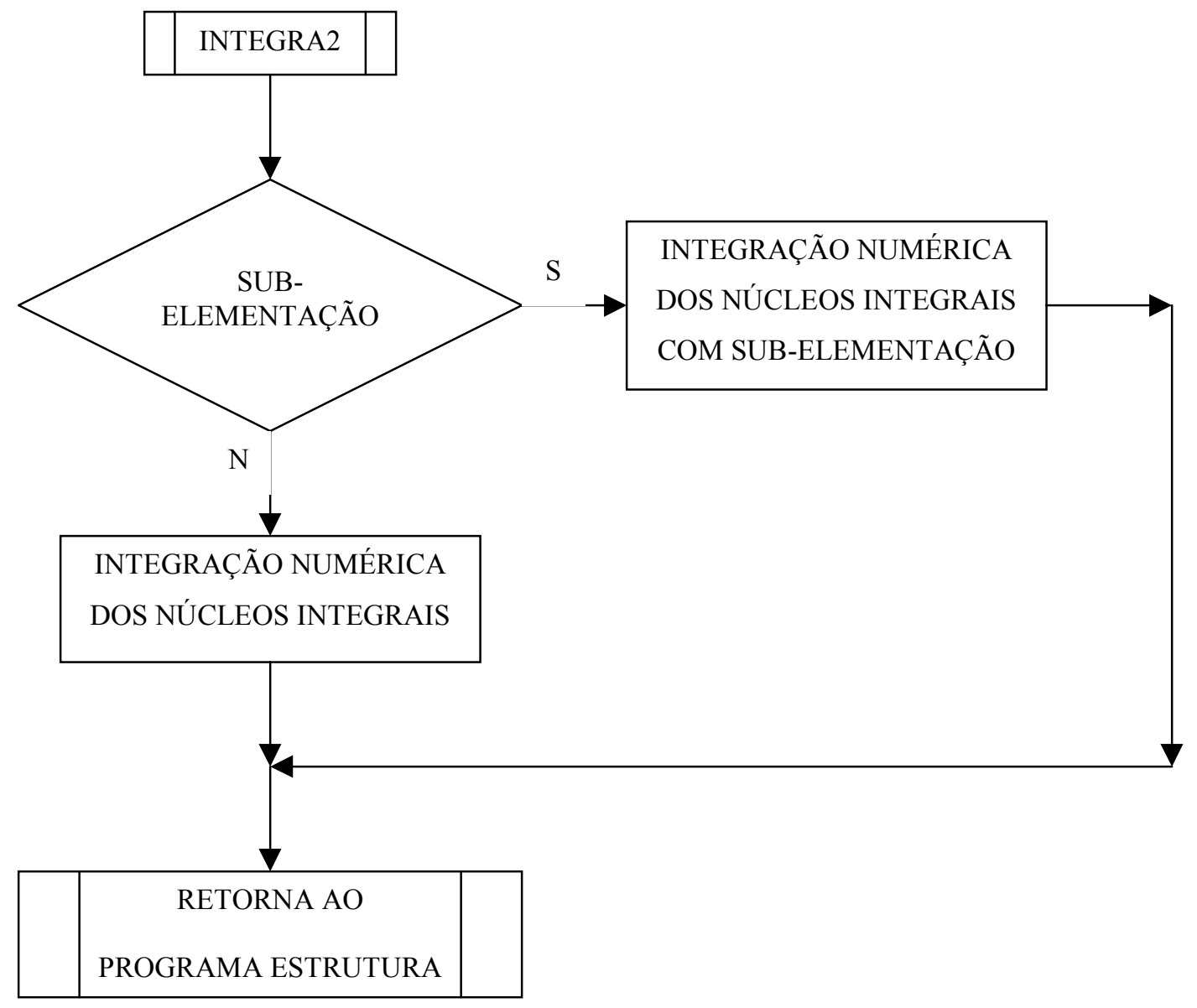




\section{ANEXO C}

\section{Distância de um ponto à reta diretriz de um elemento linear de contorno}

No item 2.4.3 do capítulo 2 foi apresentada uma técnica de integração com divisão dos elementos de contorno em sub-elementos. O cálculo dos tamanhos dos sub-elementos é baseado na distância $d$ do ponto de colocação $S$ à reta diretriz do elemento de contorno a ser integrado (figura 2.9).

Chamando de $P$ um ponto pertencente à reta diretriz $(r)$ do elemento, de maneira que a distância de $S$ à $P$ seja igual a $d$, menor distância do ponto $S$ à reta $r$.

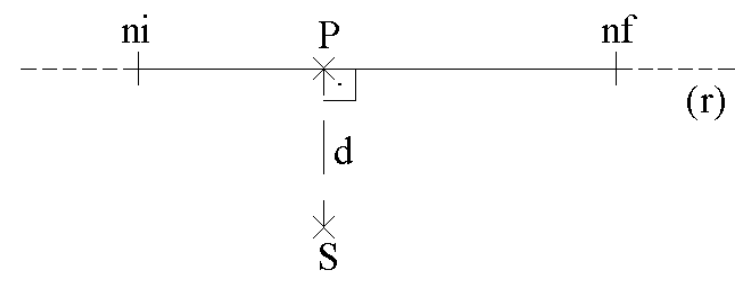

Figura C.1 - Distância $d$ do ponto $S$ à reta $(r)$

Inicialmente, calcula-se a posição do ponto $P$ no sistema de coordenada cartesiano:

$$
\begin{gathered}
\Delta X=X_{n f}-X_{n i} \quad \Delta Y=Y_{n f}-Y_{n i} \\
c 1=\Delta X \cdot X_{S}+\Delta Y \cdot Y_{S} \\
c 2=Y_{n i}-\frac{\Delta Y}{\Delta X} \cdot X_{n i}=Y_{n f}-\frac{\Delta Y}{\Delta X} \cdot X_{n f} \\
X_{P}=\frac{(c 1-\Delta Y \cdot c 2) \cdot \Delta X}{\Delta Y^{2}+\Delta X^{2}} \quad Y_{P}=\frac{\left(\Delta Y \cdot c 1-\Delta X^{2} \cdot c 2\right)}{\Delta Y^{2}+\Delta X^{2}}
\end{gathered}
$$

Em seguida pode-se calcular a distância $d$ pela fórmula abaixo:

$$
d=\sqrt{\left(X_{P}-X_{S}\right)^{2}+\left(Y_{P}-Y_{S}\right)^{2}}
$$




\section{ANEXO D}

\section{Noções sobre notação indicial}

Notação indicial é aquela que utiliza números inteiros como índices para representar variáveis, equações e operações. Em uma equação o índice pode ser de dois tipos:

(a) Índice livre: muda a equação, representa um conjunto de equações.

(b) Índice mudo: não muda a equação, opera apenas na própria equação.

$\mathrm{Na}$ equação de equilíbrio estático da Teoria da Elasticidade $\sigma_{i j, j}+b_{i}=0$, o índice $i$ é livre (representa 3 equações no caso 3D) e $j$ é mudo (representa 3 componentes de tensão em cada equação de $i$ ).

A convenção de somatório significa que quando houver um índice repetido em um termo de uma expressão, acontece uma soma. Por exemplo:

$$
\begin{gathered}
x_{i} \cdot y_{i}=k \quad(i=1,2) \quad \Rightarrow \quad x_{1} \cdot y_{1}+x_{2} \cdot y_{2}=k \\
a_{j j}=0 \quad(j=1,2,3) \quad \Rightarrow \quad a_{11}+a_{22}+a_{33}=0
\end{gathered}
$$

Percebe-se que nos exemplos (D.1) $i$ e $j$ são índices mudos. Um exemplo diferente, onde não existe a convenção de somatório, pode ser ilustrado pelo conjunto de equações $x_{i}+y_{i}=0$ com $i=1,2$. Neste caso o índice $i$ é livre.

$$
\begin{aligned}
& x_{1}+y_{1}=0 \\
& x_{2}+y_{2}=0
\end{aligned}
$$

$\mathrm{O}$ delta de Kronecker $\left(\delta_{i j}\right)$ é um símbolo usado para encurtar e simplificar equações; é definido por:

$$
\delta_{i j}=\left\{\begin{array}{lll}
1 & \text { se } & i=j \\
0 & \text { se } & i \neq j
\end{array}\right.
$$

As derivadas são representadas por vírgulas na notação indicial e o índice depois da vírgula expressa em qual direção é calculada a derivada. 


$$
\begin{gathered}
F_{i},{ }_{j}=\frac{\partial F_{i}}{\partial x_{j}} \\
\phi,_{i j}=\frac{\partial^{2} \phi}{\partial x_{i} \partial x_{j}}
\end{gathered}
$$

Quando a derivada é em calculada relação ao tempo, utilizam-se pontos sobre a grandeza a ser derivada.

$$
\begin{gathered}
\dot{\vec{u}}=\frac{\partial \vec{u}}{\partial t} \\
\ddot{\vec{u}}=\frac{\partial^{2} \vec{u}}{\partial t^{2}} \\
\dot{\phi}_{i}=\frac{\partial \phi_{i}}{\partial t} \\
\dot{F}_{i},_{j}=\frac{\partial^{2} F_{i}}{\partial x_{j} \partial t}
\end{gathered}
$$

A convenção de somatório também é válida em fórmulas diferenciais.

$$
F_{i},,_{i}=\frac{\partial F_{i}}{\partial x_{i}}=\frac{\partial F_{1}}{\partial x_{1}}+\frac{\partial F_{2}}{\partial x_{2}}+\frac{\partial F_{3}}{\partial x_{3}} \quad(i=1,2,3)
$$




\section{ANEXO E}

\section{Transformação de coordenadas}

A transformação de coordenadas utilizada neste trabalho é uma rotação, segundo o ângulo $\alpha$, do sistema de coordenadas $X Y$ para o sistema $\Gamma \eta$.

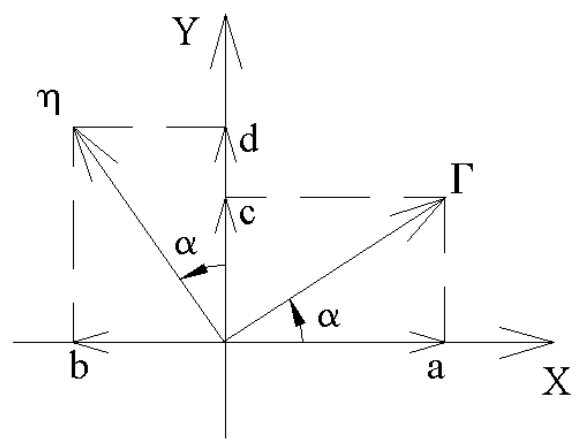

Figura E.1 - Sistemas de coordenadas

Utilizando-se as projeções dos eixo $\Gamma$ e $\eta$ em $X$ e $Y$ é possível obter um sistema linear que relaciona as coordenadas dos dois sistemas.

$$
\begin{gathered}
\left\{\begin{array}{l}
X=a-b \\
Y=c+d
\end{array}\right. \\
\left\{\begin{array}{l}
X=\cos \alpha \cdot \Gamma-\operatorname{sen} \alpha \cdot \eta \\
Y=\operatorname{sen} \alpha \cdot \Gamma+\cos \alpha \cdot \eta
\end{array}\right.
\end{gathered}
$$

A matriz de transformação $[\beta]$ é obtida através dos coeficientes do sistema linear (E.1).

$$
[\beta]=\left[\begin{array}{cc}
\cos \alpha & -\operatorname{sen} \alpha \\
\operatorname{sen} \alpha & \cos \alpha
\end{array}\right]
$$




\section{REFERÊNCIAS BIBLIOGRÁFICAS}

ABRAMOWITZ, M.; STEGUN, I.A. (1972). Handbook of mathematical functions. New York, Dover Publications.

AMINI, S.; CHEN KE; HARRIS, P.J. (1990). Iterative solution of boundary element equations for the exterior Helmholtz problem. Transactions of the ASME: Journal of vibration and acoustics, v.112, p.257-62.

BARRETTO, S.F.A.; MESQUITA NETO, E.; ANTES, H. (1998). A 2D fluid-structure coupling, using boundary element formulation. Proceedings of the modeling and simulation based engineering, v.1, p.148-53.

BREBBIA, C.A.; DOMINGUEZ, J. (1992). Boundary elements (an introductory course). 2.ed. Southampton, Computational Mechanics Publications.

BROD, K. (1984). On the uniqueness of solution for all wavenumbers in acoustic radiation. The journal of the acoustical society of America, v.76, n.4, p.1238-43.

BURTON, A.J.; MILLER, G.F. (1971). The application of integral equation methods to the numerical solution of some exterior boundary-value problems. Proceedings of the royal society of London, Series A - mathematical and physical sciences, v.323, p.201-10.

CISKOWSKI, R.D.; BREBBIA, C.A. (1991). Boundary element methods in acoustics. Southampton, Computational Mechanics Publications.

COPLEY, L.G. (1968). Fundamental results concerning representations in acoustic radiation. The journal of the acoustical society of America, v.44, n.1, p.28-32.

COPLEY, L.G. (1967). Integral equation method for radiation from vibrating bodies. The journal of the acoustical society of America, v.41, n.4, p.807-16.

COSTA JUNIOR, J.A. (1988). An alternative procedure to solve Helmholtz problem by BEM. In: BREBBIA, C.A., ed. Boundary elements X. Berlin, Springer-Verlag. v.4, s.3, p.245-53.

CUNEFARE, K.A.; KOOPMAN, G.; BROD, K. (1989). A boundary element method for acoustic radiation valid for all wavenumbers. The journal of the acoustical society of America, v.85, n.1, p.39-48. 
DOMINGUEZ, J. (1993). Boundary elements in dynamics. Southampton, Computational Mechanics Publications.

FRIEDMAN, M.B.; SHAW, R. (1962). Diffraction of pulses by cylindrical obstacles of arbitrary cross section. Transactions of the ASME: Journal of applied mechanics, v.29, p.40-6.

HAYASHI, Y. (1973). The Dirichlet problem for the two-dimensional Helmholtz equation for an open boundary. Journal of mathematical analysis and applications, v.44, p.489530 .

JASWON, M.A. (1963). Integral equation methods in potential theory I. Proceedings of the royal society of London, Series A - mathematical and physical sciences, v.275, p.23-32.

KANE, J.H. (1994). Boundary element analysis in engineering continuum mechanics. New Jersey, Prentice Hall.

KINSLER, L.E.; FREY, A.R.; COPPENS, A.B.; SANDERS, J.V. (1982). Fundamentals of acoustics. 3.ed. New York, John Wiley \& Sons.

KLEINMAN, R.E.; ROACH, G.F. (1974). Boundary integral equations for the threedimensional Helmholtz equation. SIAM review, v.16, n.2, p.214-36.

LACERDA, L.A.; WROBEL, L.C.; MANSUR, W.J. (1996). A boundary integral formulation for two-dimensional acoustic radiation in a subsonic flow. The journal of the acoustical society of America, v.100, n.1, p.98-107.

LEWIS, R.W.; BETTESS, P.; HINTON, E. (1984). Numerical Methods in Coupled Systems. In: ZIENKIEWICZ, O.C., Coupled Problems and their Numerical Solution. Great Britain, John Wiley \& Sons. p.35-58.

MEYER, W.L.; BELL, W.A; STALLYBRASS, M.P.; ZINN, B.T. (1979). Prediction of the sound field radiated from axisymmetric surfaces. . The journal of the acoustical society of America, v.65, n.3, p.631-8.

MEYER, W.L.; BELL, W.A.; ZINN, B.T.; STALLYBRASS, M.P. (1978). Boundary integral solutions of three dimensional acoustic radiation problems. Journal of sound and vibration, v.59, n.2, p.245-62.

MITZNER, K.M. (1967). Numerical solution for transient scattering from a hard surface of arbitrary shape - retarded potential technique. The journal of the acoustical society of America, v.42, n.2, p.391-7.

MORAND, H.J.-P.; OHAYON, R. (1995). Fluid structure interaction: applied numerical methods. Paris, Masson.

NEPOMUCENO, L.X. (1977). Acústica. São Paulo, Editora Edgard Blücher. 
PIASZCYK, C.M.; KLOSNER, J.M. (1984). Acoustic radiation from vibrating surfaces at characteristic frequencies. The journal of the acoustical society of America, v.75, n.2, p.363-75.

PIPES, L.A.; HARVILL, L.R. (1970). Applied mathematics for engineers and physicists. 3.ed. Tokyo, McGraw-Kogakusha.

RIZZO, F.J. (1967). An integral equation approach to boundary value problems of classical elastostatics. Quarterly of applied mathematics / American mathematical society, v.XXV, n.1, p.83-95.

SCHENCK, H.A. (1968). Improved integral formulation for acoustic radiation problems. The journal of the acoustical society of America, v.44, n.1, p.41-58.

SEYBERT, A.F.; RENGARAJAN, T.K. (1987). The use of CHIEF to obtain unique solutions for acoustic radiation using boundary integral equations. The journal of the acoustical society of America, v.81, n.5, p.1299-306.

SEYBERT, A.F.; SOENARKO, B.; RIZZO, F.J.; SHIPPY, D.J. (1986). A special integral equation formulation for acoustic radiation and scattering for axisymmetric bodies and boundary conditions. The journal of the acoustical society of America, v.80, n.4, p.12417.

SEYBERT, A.F.; SOENARKO, B.; RIZZO, F.J.; SHIPPY, D.J. (1985). An advanced computational method for radiation and scattering of acoustic waves in three dimensions. The journal of the acoustical society of America, v.77, n.2, p.362-8.

SHAW, R.P. (1968). Retarded potential approach to the scattering of elastic pulses by rigid obstacles of arbitrary shape. The journal of the acoustical society of America, v.44, n.3, p.745-8.

SYMM, G.T. (1963). Integral equation methods in potential theory II. Proceedings of the royal society of London, Series A - mathematical and physical sciences, v.275, p.33-46.

WATERMAN, P.C. (1969). New formulation of acoustic scattering. The journal of the acoustical society of America, v.45, n.6, p.1417-29.

WILTON, D.T. (1978). Acoustic radiation and scattering from elastic structures. International journal for numerical methods in engineering, v.13, p.123-38.

WU, T.W.; LEE, L. (1994). A direct boundary integral formulation for acoustic radiation in a subsonic uniform flow. Journal of sound and vibration, v.175, n.1, p.51-63.

YANG, S.A. (1999). A boundary integral equation method for two-dimensional acoustic scattering problems. The journal of the acoustical society of America, v.105, n.1, p.93105. 
YOON, W.S.; PARK, J.M.; EVERSMAN, W. (1990). Two-dimensional radiation and scattering at short wave length. Transactions of the ASME: Journal of vibration and acoustics, v.112, p.384-91. 


\section{BIBLIOGRAFIA COMPLEMENTAR}

ANZOU, L.A. (1996). Formulação do método dos elementos de contorno para equação de Helmholtz, visando modelagem da radiação e dispersão acústica. Campinas. 171p. Dissertação (Mestrado) - Faculdade de Engenharia Mecânica, Universidade Estadual de Campinas.

CODA, H.B. (1990). Análise da vibração livre de meios elásticos bidimensionais pelo método dos elementos de contorno. São Carlos. 130p. Dissertação (Mestrado) - Escola de Engenharia de São Carlos, Universidade de São Paulo.

DEMMEL, J.W. (1993). Berkeley lecture notes on numerical linear algebra. Mathematics department and computer science division, University of California.

MORSE, P.M. (1948). Vibration and sound. 2.ed. New York, McGraw-Hill.

MORSE, P.M.; INGARD, K.U. (1968). Theoretical acoustics. New York, McGraw-Hill.

SILVA, J.J.R. (1994). Acoustic and elastic wave scattering using boundary elements. Southampton, Computational Mechanics Publications.

SPIEGEL, M.R. (1992). Manual de fórmulas, métodos e tabelas de matemática. 2.ed. São Paulo, Makron Books. (Coleção Schaum)

VENTURINI, W.S. (1988). Um estudo sobre o método dos elementos de contorno e suas aplicações em problemas de engenharia. São Carlos. 348p. Tese (Livre-Docência) Escola de Engenharia de São Carlos, Universidade de São Paulo.

WYLIE, C.R.; BARRETT, L.C. (1985). Advanced engineering mathematics. 5.ed. London, McGraw-Hill. 


\section{APÊNDICE}

\section{Escala Decibel}

A escala Decibel representa as pressões acústicas em uma escala logarítmica. O nivel de pressão acústica NPS representa a pressão acústica $p$ nesta escala.

$$
N P S=20 \cdot \log _{10}\left(\frac{p}{p_{R E F}}\right)
$$

Onde:

$p_{R E F}$ é a pressão acústica de referência no meio. Para o ar, $p_{R E F}=20 \cdot 10^{-6} P a$.

A unidade de NPS é expressa em decibels $[d B]$.

A escala Decibel é útil para comprimir o intervalo de pressões acústicas encontrado na natureza, que em outras escalas seria extenso e de difícil avaliação em termos comparativos. Outro motivo para a utilização da escala Decibel é o fato do julgamento humano da intensidade entre dois sons atender um comportamento logarítmico, segundo KINSLER et al. (1982). 